\title{
A WEAK CONVERGENCE APPROACH TO INVENTORY CONTROL USING A LONG-TERM AVERAGE CRITERION
}

\author{
K. L. HELMES, ${ }^{*}$ Humboldt University of Berlin \\ R. H. STOCKBRIDGE ${ }^{* * * * *}$ AND \\ C. ZHU, ${ }^{* * * * * *}$ University of Wisconsin-Milwaukee
}

\begin{abstract}
In this paper we continue the examination of inventory control in which the inventory is modeled by a diffusion process and a long-term average cost criterion is used to make decisions. The class of such models under consideration has general drift and diffusion coefficients, and boundary points that are consistent with the notion that demand should tend to reduce the inventory level. The conditions on the cost functions are greatly relaxed from those in Helmes et al. (2017). Characterization of the cost of a general $(s, S)$ policy as a function of two variables naturally leads to a nonlinear optimization problem over the ordering levels $s$ and $S$. Existence of an optimizing pair $\left(s_{*}, S_{*}\right)$ is established for these models under very weak conditions; nonexistence of an optimizing pair is also discussed. Using average expected occupation and ordering measures and weak convergence arguments, weak conditions are given for the optimality of the $\left(s_{*}, S_{*}\right)$ ordering policy in the general class of admissible policies. The analysis involves an auxiliary function that is globally $C^{2}$ and which, together with the infimal cost, solves a particular system of linear equations and inequalities related to but different from the longterm average Hamilton-Jacobi-Bellman equation. This approach provides an analytical solution to the problem rather than a solution involving intricate analysis of the stochastic processes. The range of applicability of these results is illustrated on a drifted Brownian motion inventory model, both unconstrained and reflected, and on a geometric Brownian motion inventory model under two different cost structures.
\end{abstract}

Keywords: Inventory; impulse control; long-term average cost; general diffusion model; $(s, S)$ policy; weak convergence

2010 Mathematics Subject Classification: Primary 93E20

Secondary 90B05

\section{Introduction}

In this paper we further develop our examination of the long-term average cost criterion for inventory models of diffusion type in which the only control over the inventory levels is through the action of ordering additional stock; see Helmes et al. (2017) for our previous investigation. It identifies weak sufficient conditions for optimality of an $(s, S)$ ordering policy in the general

Received 30 January 2017; revision received 14 September 2018.

The supplementary material for this article can be found at http://doi.org/10.1017/apr.2018.50.

* Postal address: Institute for Operations Research, Humboldt University of Berlin, Spandauer Street 1, 10178, Berlin, Germany. Email address: helmes@wiwi.hu-berlin.de

** Postal address: Department of Mathematical Sciences, University of Wisconsin-Milwaukee, Milwaukee, WI 53201, USA.

*** Email address: stockbri@uwm.edu

**** Email address: zhu@uwm.edu 
class of admissible policies as well as providing some sufficient conditions for nonexistence of an optimal $(s, S)$ policy.

We model the inventory processes (in the absence of orders) as solutions to a stochastic differential equation

$$
\mathrm{d} X_{0}(t)=\mu\left(X_{0}(t)\right) \mathrm{d} t+\sigma\left(X_{0}(t)\right) \mathrm{d} W(t), \quad X_{0}(0)=x_{0},
$$

taking values in an interval $\ell=(a, b)$; negative values of $X_{0}(t)$ represent back-ordered inventory. The detailed discussion in Chen et al. (2010) indicates the validity of state-dependent diffusion models for inventory management.

An ordering policy $(\tau, Y)$ is a sequence of pairs $\left\{\left(\tau_{k}, Y_{k}\right): k \in \mathbb{N}\right\}$ in which $\tau_{k}$ denotes the (random) time at which the $k$ th order is placed and $Y_{k}$ denotes its size. Since order $k+1$ cannot be placed before order $k,\left\{\tau_{k}: k \in \mathbb{N}\right\}$ is an increasing sequence of times. The inventory level process $X$ resulting from an ordering policy $(\tau, Y)$ therefore satisfies the equation

$$
X(t)=x_{0}+\int_{0}^{t} \mu(X(s)) \mathrm{d} s+\int_{0}^{t} \sigma(X(s)) \mathrm{d} W(s)+\sum_{k=1}^{\infty} \mathbf{1}_{\left\{\tau_{k} \leq t\right\}} Y_{k} .
$$

Note the initial inventory level $X(0-)=x_{0}$ may be such that an order is placed at time 0 , resulting in a new inventory level at time 0 ; this possibility occurs when $\tau_{1}=0$. Also, observe that $X\left(\tau_{k}-\right)$ is the inventory level just prior to the $k$ th order being placed, while $X\left(\tau_{k}\right)$ is the level with the new inventory. Thus, this model assumes that orders are filled instantaneously.

Let $(\tau, Y)$ be an ordering policy, and let $X$ be the resulting inventory level process satisfying (1.2). Let $c_{0}$ and $c_{1}$ denote the holding/back-order cost rate and ordering cost functions, respectively. We assume there is some constant $k_{1}>0$ such that $c_{1} \geq k_{1}$; this constant represents the fixed cost for placing each order. The long-term average expected holding/backorder plus ordering costs is

$$
J_{0}(\tau, Y):=\limsup _{t \rightarrow \infty} t^{-1} \mathbb{E}\left[\int_{0}^{t} c_{0}(X(s)) \mathrm{d} s+\sum_{k=1}^{\infty} \mathbf{1}_{\left\{\tau_{k} \leq t\right\}} c_{1}\left(X\left(\tau_{k}-\right), X\left(\tau_{k}\right)\right)\right] .
$$

The goal is to identify an admissible ordering policy so as to minimize the cost.

As mentioned earlier, we revisit the problem examined in Helmes et al. (2017). We thus refer the reader to that paper for a discussion of the existing literature related to this problem; see also Bensoussan (2011). In this paper we relax the assumptions imposed on $c_{1}$, such as the concavity requirement in Yao et al. (2015). We focus the remainder of our comments on that which distinguishes this paper from our earlier publication.

The present paper differs from Helmes et al. (2017) in three significant respects: solution approach, technical requirements, and generality of the class of admissible policies. Here, we employ weak convergence of the average expected occupation and ordering measures to analyze the long-term average costs, whereas Helmes et al. (2017) relied heavily on intricate pathwise analysis of the stochastic inventory processes. The benefit of the weak convergence approach lies in the ease of establishing the tightness of the expected occupation measures and, hence, their sequential compactness. This compactness ensures the existence of long-term average limiting measures that are key to the analysis of the costs. Quite surprisingly, tightness is not required of the average expected ordering measures, so there may not exist any limiting ordering measures. Nevertheless, a limiting argument establishes optimality in the general 
class of admissible policies of an $\left(s_{*}, S_{*}\right)$ policy. In Appendix C we present an example of an ordering policy having finite cost for which the average expected occupation measures are tight, but the average expected ordering measures are not tight.

From a technical point of view, the weak convergence approach allows conditions on both cost functions $c_{0}$ and $c_{1}$ to be considerably relaxed by removing many structural requirements (compare Condition 2.2 below with Condition 2.3 of Helmes et al. (2017)). For example, the requirement that $c_{0}$ approaches $\infty$ at each boundary and the rather restrictive modularity condition (2.6) of that paper on $c_{1}$ are unnecessary. In their places, we merely require that these functions be continuous, with $c_{0}$ continuous at the boundaries, an integrability condition on $c_{0}$ at the boundary $b$, as well as other boundedness relations contained in Conditions 2.3 and 5.1.

Most significantly, the two papers differ in how the auxiliary function is defined, which, together with the infimal cost, form a solution of a particular system of linear equations and inequalities (see Proposition 4.1) related to but different from the long-term average HamiltonJacobi-Bellman equation. In the present paper, the auxiliary function $G_{0}$ is a $C^{2}$ function defined globally on $\ell$, contrasting with the function $G$ in the earlier paper in which $C^{1}$-smoothly pastes two functions at a significant point in $\ell$. Moreover, frequently in the literature, the longterm average auxiliary function is obtained from the value functions of discounted problems using the method of vanishing discount. The function $G_{0}$ in this paper is not obtained in this manner.

The functions $G$ and $G_{0}$ in the two papers play a central role in establishing general optimality of an $(s, S)$ ordering policy. In this paper we provide an analytic solution for the general model that applies to all admissible policies. In contrast, in Helmes et al. (2017) we showed optimality in a smaller class of ordering policies for the general models under a more restrictive set of conditions. Optimality of $(s, S)$ policies for the general class of admissible policies for two examples from He et al. (2017) and Helmes et al. (2017) was established using ad hoc methods specifically tuned to the examples.

The initial steps to the solution of the inventory control problem are similar to those in Helmes et al. (2017). In the next section we briefly develop the model formulation, identify two important functions, and analyze $(s, S)$ ordering policies with an emphasis on the differences between the papers. In particular, we concentrate on the more relaxed conditions in the current paper and we provide some new results that are required for the weak convergence analysis. Some results are common to both papers; we refer to Helmes et al. (2017) for those results whose proofs remain valid, but we provide a complete proof in Appendix A for Theorem 2.1 since the previous proof is no longer valid for the general models of this paper. Section 2 is designed to briefly set some of the common foundations in the two papers so as to allow the reader to quickly access the new ideas and approaches in succeeding sections of this paper. In Section 3 we introduce the expected occupation and expected ordering measures, and discuss their tightness (or not).

The main contributions of this paper are contained in Sections 4 and 5. In Section 4 we define a particular auxiliary function $G_{0}$ at the heart of our analytical approach and give two important results related to $G_{0}$. In Section 5 we then approximate $G_{0}$ by functions in a subclass and use these approximations to establish optimality of the $\left(s_{*}, S_{*}\right)$ ordering policy in the general class of admissible policies. Finally, in Section 6 we illustrate the ease of application of these results on examples involving a drifted Brownian motion, both unconstrained and with reflection at $\{0\}$, and a geometric Brownian motion having two different cost structures. These examples are chosen to illustrate the more general applicability of this paper's results over those in Helmes et al. (2017). 


\section{Formulation and preliminary results}

In this section we briefly establish the models under consideration, which are more general than those in Helmes et al. (2017). The approach is very similar to that taken in Sections 2 and 3 of that paper, so we refer the reader to those sections for more details. We emphasize the differences between the conditions. Some proofs must be modified for the more general models of this paper. Also, some new results are required for our weak convergence arguments. This overview will be kept brief so that the reader may reach the new ideas of this paper with a minimum of effort. The reader may also find Chapter 15 of Karlin and Taylor (1981) to be a good reference for the boundary classifications of one-dimensional diffusions, especially for properties of the scale function and speed measure.

\subsection{Formulation}

Let $\ell=(a, b) \subseteq \mathbb{R}$. In the absence of ordering, the inventory process $X_{0}$ satisfies (1.1) and is a regular diffusion. Throughout the paper, we assume that the functions $\mu$ and $\sigma$ are continuous on $\ell$, and that (1.1) is nondegenerate. The initial position of $X_{0}$ is taken to be $x_{0}$ for some $x_{0} \in \ell$. Let $\left\{\mathcal{F}_{t}\right\}$ denote the filtration generated by $X_{0}$, augmented so that it satisfies the usual conditions. We place the following assumptions on the underlying diffusion model.

Condition 2.1. (a) Both the speed measure $M$ and the scale function $S$ of the process $X_{0}$ are absolutely continuous with respect to Lebesgue measure.

(b) The left boundary $a$ is attracting and the right boundary $b$ is nonattracting. Moreover, when $b$ is a natural boundary, $M[y, b)<\infty$ for each $y \in \ell$. The boundaries $a=-\infty$ and $b=\infty$ are required to be natural.

When $b$ is a natural boundary, the requirement that $M[y, b)<\infty$ for each $y \in \ell$ is imposed in this paper as this is required for our general approach and does not hold in general. Lemma 2.4 of Helmes et al. (2017) shows that this condition follows from the more restrictive assumptions in that paper.

Associated with the scale function $S$ of Condition 2.1, we can define the scale measure on the Borel sets of $\ell$ by $S[y, z]=S(z)-S(y)$ for $[y, z] \subset \ell$.

From the modeling point of view, Condition 2.1(b) is reasonable since it essentially says that, in the absence of ordering, demand tends to reduce the size of the inventory. The boundary point $a$ may be regular, exit, or natural with $a$ being attainable in the first two cases and unattainable in the third. In the case that $a$ is a regular boundary, its boundary behavior must also be specified as being either reflective or sticky. The boundary point $b$ is either natural or entrance and is unattainable from the interior in both cases. Following the approach in Helmes et al. (2017), we define the state space of possible inventory levels to be the interval $\mathscr{E}$ which excludes any natural boundary point, and includes $a$ when it is attainable and $b$ when it is entrance. Since orders typically increase the inventory level, define $\mathcal{R}=\left\{(y, z) \in \mathcal{E}^{2}: y<z\right\}$ in which $y$ denotes the pre-order and $z$ the post-order inventory levels, respectively.

Since we are using weak convergence methods for measures on $\mathcal{E}$ and $\mathcal{R}$, we will need their closures as well. Define $\overline{\mathcal{E}}$ to be the closure in $\mathbb{R}$ of $\mathcal{E}$; thus, when a boundary is finite and natural, it is not an element of $\mathcal{E}$ but is in $\overline{\mathcal{E}}$. Note that $\pm \infty \notin \overline{\mathcal{E}}$. Also, set $\overline{\mathcal{R}}=\left\{(y, z) \in \mathcal{E}^{2}: y \leq z\right\}$; in contrast to $\mathcal{R}$, the set $\overline{\mathcal{R}}$ includes orders of size 0 . Note the subtle distinction between $\overline{\mathcal{E}}$, which includes boundaries that are finite and natural, and $\overline{\mathcal{R}}$, which does not allow either coordinate to be such a point. The reason for this distinction lies in the fact that the expected ordering measures are not required to be tight. 
We adopt the standard assumptions on the class $\mathcal{A}$ of admissible ordering policies; namely that $\left\{\tau_{k}: k \in \mathbb{N}\right\}$ is an increasing sequence of $\left\{\mathcal{F}_{t}\right\}$-stopping times and, for each $k \in \mathbb{N}, Y_{k}$ is nonnegative, $\mathcal{F}_{\tau_{k}}$-measurable, and satisfies $X\left(\tau_{k}\right) \in \mathcal{E}$.

Turning to the cost functions, we impose the following standing assumptions throughout the paper.

Condition 2.2. (a) The holding/back-order cost function $c_{0}: \ell \rightarrow \mathbb{R}^{+}$is continuous. Moreover, at the boundaries

$$
\lim _{x \rightarrow a} c_{0}(x)=: c_{0}(a) \text { exists in } \overline{\mathbb{R}^{+}} \text {and } \lim _{x \rightarrow b} c_{0}(x)=: c_{0}(b) \text { exists in } \overline{\mathbb{R}^{+}}
$$

we require $c_{0}( \pm \infty)=\infty$. Finally, for each $y \in \ell$,

$$
\int_{y}^{b} c_{0}(v) \mathrm{d} M(v)<\infty
$$

(b) The function $c_{1}: \overline{\mathcal{R}} \rightarrow \overline{\mathbb{R}^{+}}$is in $C(\overline{\mathcal{R}})$ with $c_{1} \geq k_{1}>0$ for some constant $k_{1}$.

Again, since we will use weak convergence arguments for measures on $\overline{\mathcal{E}}$, we require $c_{0}$ to be continuous at the boundaries, even when they are natural. The models in Helmes et al. (2017) impose the stricter condition that $c_{0}(a)=c_{0}(b)=\infty$. Condition (2.1) is required in both papers, but Helmes et al. (2017) also imposed $\int_{y}^{b} \int_{u}^{b} c_{0}(v) \mathrm{d} M(v) \mathrm{d} S(u)=\infty$ for each $y \in \ell$. Thus, with the view for applications, the current paper allows a more flexible class of functions $c_{0}$. Both papers assume that $c_{1}$ is continuous and strictly bounded away from 0 . Helmes et al. (2017) also imposed on $c_{1}$ monotonicity $\left(c_{1}(y, z) \geq c_{1}(z, z)\right.$ for $\left.y \leq z\right)$ and (restrictive) modularity $\left(c_{1}(w, z)+c_{1}(x, y)=c_{1}(w, y)+c_{1}(x, z)\right.$ for $\left.w \leq x \leq y \leq z\right)$.

Both papers assume that $c_{0}$ is inf-compact; that is, $\left\{x \in \overline{\mathcal{E}}: c_{0}(x) \leq k\right\}$ is compact for each $k \geq 0$. Though this is not explicitly stated in Condition 2.2(a), it is immediate when $c_{0}(a)=\infty$ and $c_{0}(b)=\infty$. When $c_{0}(a)<\infty$ and $c_{0}(b)<\infty, a$ and $b$ must be finite. Continuity of $c_{0}$, including at the boundaries, then establishes that $\left\{x \in \overline{\mathcal{E}}: c_{0}(x) \leq k\right\} \cap[a, b]$ is compact for every $k \geq 0$.

The generator of the process $X$ between jumps (corresponding to the diffusion $X_{0}$ ) is $A f=$ $\frac{1}{2} \sigma^{2} f^{\prime \prime}+\mu f^{\prime}$, which is defined for all $f \in C^{2}(\ell)$ or, equivalently,

$$
A f=\frac{1}{2} \frac{\mathrm{d}}{\mathrm{d} M}\left(\frac{\mathrm{d} f}{\mathrm{~d} S}\right)
$$

To capture the effect ordering has on the inventory process, define the jump operator $B: C(\mathcal{E}) \rightarrow$ $C(\overline{\mathcal{R}})$ by $B f(y, z)=f(z)-f(y)$ for $(y, z) \in \overline{\mathcal{R}}$.

\subsection{Important functions}

As in Helmes et al. (2017), two functions play a central role in our search for an optimal ordering policy. Using the initial position $x_{0} \in \ell$, define the functions $g_{0}$ and $\zeta$ on $\ell$ by

$$
g_{0}(x):=\int_{x_{0}}^{x} \int_{u}^{b} 2 c_{0}(v) \mathrm{d} M(v) \mathrm{d} S(u) \text { and } \zeta(x):=\int_{x_{0}}^{x} \int_{u}^{b} 2 \mathrm{~d} M(v) \mathrm{d} S(u),
$$

and extend these functions to $\overline{\mathcal{E}}$ by continuity. Observe that both $g_{0}$ and $\zeta$ are negative on $\left(a, x_{0}\right)$ and positive on $\left(x_{0}, b\right)$; also, $g_{0}$ may take values $\pm \infty$ at the boundaries. The definitions of $g_{0}$ and $\zeta$ differ slightly from the similar functions in Helmes et al. (2017) in that we specify 
the lower limit of the outer integrals to be the initial point $x_{0}$. Using the second characterization of $A$, it immediately follows that $g_{0}$ and $\zeta$, respectively, are particular solutions on $\ell$ of

$$
A f=-c_{0}, \quad f\left(x_{0}\right)=0 \quad \text { and } \quad A f=-1, \quad f\left(x_{0}\right)=0 .
$$

Other solutions to these differential equations having value 0 at $x_{0}$ include summands of the form $K\left(S(x)-S\left(x_{0}\right)\right), K \in \mathbb{R}$, since the constant function and the scale function $S$ are linearly independent solutions of the homogeneous equation $A f=0$. However, such additional terms grow too quickly near the boundary $b$, so a transversality condition in Proposition 4.2 fails (see Remark 4.2 below) and, therefore, the definitions of $g_{0}$ and $\zeta$ in (2.2) exclude these terms.

To gain some intuition for the functions $g_{0}$ and $\zeta$, let $(y, z) \in \mathcal{R}$ and let $X_{0}$ satisfy (1.1) with $X_{0}(0)=z$. Define $\tau_{y}=\inf \left\{t \geq 0: X_{0}(t)=y\right\}$. Then

$$
\mathbb{E}_{z}\left[\int_{0}^{\tau_{y}} c_{0}\left(X_{0}(s)\right) \mathrm{d} s\right]=g_{0}(z)-g_{0}(y) \quad \text { and } \quad \mathbb{E}_{z}\left[\tau_{y}\right]=\zeta(z)-\zeta(y) .
$$

This result is contained in Proposition 2.6 of Helmes et al. (2017), whose proof requires only Condition 2.1(b) and (2.1) of Condition 2.2(a) in this paper. Thus, these relationships continue to hold for the models in this paper.

The proof of Proposition 3.5 in Helmes et al. (2017, pp. 1848-1849) establishes that $\lim _{y \rightarrow a} \zeta(y)>-\infty$ when $a$ is attainable (regular or exit) and $\lim _{y \rightarrow a} \zeta(y)=-\infty$ when $a$ is unattainable (natural). The proof remains valid for the models in this paper.

We now provide the first new result that arises from the possibility of finite values of $c_{0}(a)$ and $c_{0}(b)$. Its technical proof is given in Appendix A.

Lemma 2.1. Assume that Condition 2.1 holds. Suppose that a and $b$ are natural boundaries, and let $c_{0}(a)$ and $c_{0}(b)$ be as in Condition 2.2(a). Then the following asymptotic behaviours hold:

$$
\begin{array}{cc}
\lim _{y \rightarrow a} \frac{g_{0}(z)-g_{0}(y)}{\zeta(z)-\zeta(y)}=c_{0}(a) \quad \text { for all } z \in \ell, & \lim _{z \rightarrow b} \frac{g_{0}(z)-g_{0}(y)}{\zeta(z)-\zeta(y)}=c_{0}(b) \quad \text { for all } y \in \ell \\
\lim _{(y, z) \rightarrow(a, a)} \frac{g_{0}(z)-g_{0}(y)}{\zeta(z)-\zeta(y)}=c_{0}(a), & \lim _{(y, z) \rightarrow(b, b)} \frac{g_{0}(z)-g_{0}(y)}{\zeta(z)-\zeta(y)}=c_{0}(b), \\
\lim _{y \rightarrow a} \frac{g_{0}(y)}{\zeta(y)}=c_{0}(a), & \lim _{z \rightarrow b} \frac{g_{0}(z)}{\zeta(z)}=c_{0}(b) .
\end{array}
$$

These imply that $\lim _{y \rightarrow a} g_{0}(y)=-\infty$ when $c_{0}(a)>0$ and $\lim _{z \rightarrow b} g_{0}(z)=\infty$ when $c_{0}(b)>0$.

\subsection{Analysis of $(s, S)$ ordering policies}

Both this paper and Helmes et al. (2017) rely on characterizing the long-term average cost for $(s, S)$ ordering policies. For $(y, z) \in \mathcal{R}$, define the $(y, z)$ ordering policy $(\tau, Y)$ such that $\tau_{0}=0$ and

$$
\tau_{k}=\inf \left\{t>\tau_{k-1}: X(t-) \leq y\right\} \quad \text { and } \quad Y_{k}=z-X\left(\tau_{k}-\right), \quad k \geq 1,
$$

in which $X$ is the inventory level process satisfying (1.2) with this ordering policy. The above definition of $\tau_{k}$ must be slightly modified when $k=1$ to be $\tau_{1}=\inf \{t \geq 0: X(t-) \leq y\}$ to allow for the first jump to occur at time 0 when $x_{0} \leq y$. 
Theorem 2.1 of Sigman and Wolff (1993) provides existence and uniqueness of the stationary distribution for the process $X$ arising from a $(y, z)$ ordering policy for any $y, z \in \&$ with $y<z$, and, moreover, the one-dimensional distributions $\mathbb{P}(X(t) \in \cdot)$ converge weakly to the stationary distribution as $t$ tends to $\infty$. Proposition 3.1 of Helmes et al. (2017) derives the density $\pi$ of the stationary distribution for $X$ and the long-run frequency $\kappa=1 / B \zeta(y, z)$ of orders. Its proof remains valid for the models in this paper.

Proposition 3.4 of Helmes et al. (2017) uses a renewal argument to characterize the long-term average cost $J_{0}(\tau, Y)$ for the $(y, z)$ ordering policy in (2.6):

$$
\lim _{t \rightarrow \infty} \frac{1}{t} \int_{0}^{t} c_{0}(X(s)) \mathrm{d} s=\frac{B g_{0}(y, z)}{B \zeta(y, z)} \quad\left(\text { a.s. and in } L^{1}\right) ;
$$

therefore,

$$
J_{0}(\tau, Y)=\frac{c_{1}(y, z)+B g_{0}(y, z)}{B \zeta(y, z)} .
$$

The $L^{1}$ convergence is given in the proof of Proposition 3.4, though it is not stated directly in the proposition. The proposition applies to models in which $c_{0}$ is asymptotically infinite at both boundaries and $(y, z)$ is in the interior of $\mathcal{R}$. These conditions are relaxed in the current paper so $c_{0}$ may be finite at a boundary. The proof remains valid whenever $y=a>-\infty$ is attainable or $z=b<\infty$ is an entrance point (in which cases $a, b \in \mathcal{E}$ ). Note that, when $b$ is an entrance boundary, $g_{0}(b)$ is allowed to be finite or infinite.

Motivated by (2.8), define the function $F_{0}: \overline{\mathcal{R}} \rightarrow \overline{\mathbb{R}^{+}}$by

$$
F_{0}(y, z):= \begin{cases}\frac{c_{1}(y, z)+B g_{0}(y, z)}{B \zeta(y, z)}, & (y, z) \in \mathcal{R}, \\ \infty, & (y, z) \in \overline{\mathcal{R}} \text { with } y=z .\end{cases}
$$

Observe that $F_{0}$ is well defined and continuous on $\overline{\mathcal{R}}$. The function $F_{0}$ is the same as $F$ in Helmes et al. (2017), though its definition here is explicit on the boundary $y=z$ representing orders of size 0 .

The goal is to optimize $F_{0}$. Since $c_{1}>0, F_{0}(y, z)>0$ for every $(y, z) \in \overline{\mathcal{R}}$ and, thus, $\inf _{(y, z) \in \overline{\mathcal{R}}} F_{0}(y, z)=: F_{0}^{*} \geq 0$. The models in this paper allow $F_{0}^{*}=0$, in which case it immediately follows that there is no minimizing pair $\left(y_{0}^{*}, z_{0}^{*}\right)$ of $F_{0}$. A new proposition gives some sufficient conditions under which this occurs.

Proposition 2.1. Assume that Conditions 2.1 and 2.2 hold. Also, assume that any of the following conditions hold.

(a) The point $a>-\infty$ is a natural boundary and either ( $i$ ) or (ii) holds:

(i) for some $z \in \mathcal{E}, \lim \sup _{y \rightarrow a}\left(c_{1}(y, z)-g_{0}(y)\right) / \zeta(y)=0$;

(ii) $c_{0}(a)=0$ and

$$
\liminf _{(y, z) \rightarrow(a, a)} \frac{c_{1}(y, z)}{\zeta(z)-\zeta(y)}=0 .
$$

(b) The point $b<\infty$ is a natural boundary and either (i) or (ii) holds:

(i) for some $y \in \mathcal{E}, \liminf _{z \rightarrow b}\left(c_{1}(y, z)+g_{0}(z)\right) / \zeta(z)=0$;

(ii) $c_{0}(b)=0$ and $\liminf _{(y, z) \rightarrow(b, b)} c_{1}(y, z) /(\zeta(z)-\zeta(y))=0$.

Then $F_{0}^{*}=0$ and there does not exist any pair $\left(y_{0}^{*}, z_{0}^{*}\right) \in \mathcal{R}$ which minimizes $F_{0}$. 
Proof. The arguments in cases (a) and (b) are essentially the same, so we establish the result when (a) holds. Since $a$ is a natural boundary, $\zeta(y) \rightarrow-\infty$ as $y \rightarrow a$. Thus, under the hypothesis in (a)(i), for some $z \in \mathcal{E}$,

$$
0=-\limsup _{y \rightarrow a} \frac{c_{1}(y, z)-g_{0}(y)}{\zeta(y)}=\liminf _{y \rightarrow a} \frac{c_{1}(y, z)+g_{0}(z)-g_{0}(y)}{\zeta(z)-\zeta(y)}=\liminf _{y \rightarrow a} F_{0}(y, z) .
$$

Since $F_{0}(y, z)>0$ for all $(y, z) \in \mathcal{R}$, the result follows. If (a)(ii) holds then combining (2.10) with (2.4) of Lemma 2.1 establishes that $F_{0}^{*}=0$ and, again, the result holds.

As in Helmes et al. (2017), the main results depend on the existence of an optimizing pair $\left(y_{0}^{*}, z_{0}^{*}\right) \in \mathcal{R}$ of $F_{0}$. Central to the proof of this existence in Proposition 3.5 of Helmes $e t$ al. (2017) is the assumption that $c_{0}(a)=c_{0}(b)=\infty$. The relaxed assumptions on $c_{0}$ in Condition 2.2 allow us to identify a weaker set of conditions for the existence of an optimizing pair. (In Section 6 we present models which satisfy these conditions but which fail to satisfy the more restrictive conditions in Helmes et al. (2017).)

Condition 2.3. The following conditions hold.

(a) The boundary a is regular; or exit; or a is a natural boundary for which either (i) or (ii) holds:

(i) $c_{0}(a)=\infty$;

(ii) $c_{0}(a)<\infty$, the function $F_{0}(\cdot, z)$ is strictly decreasing in a neighborhood of a for each $z \in \mathcal{E}$, and there exists some $(\hat{y}, \hat{z}) \in \mathcal{R}$ such that $F_{0}(\hat{y}, \hat{z})<c_{0}(a)$.

(b) The boundary $b$ is entrance; or $b$ is natural for which either (i) or (ii) holds:

(i) $c_{0}(b)=\infty$;

(ii) $c_{0}(b)<\infty, F_{0}(y, \cdot)$ is strictly increasing in a neighborhood of b for every $y \in \mathcal{E}$, and there exists some $(\tilde{y}, \tilde{z}) \in \mathcal{R}$ such that $F_{0}(\tilde{y}, \tilde{z})<c_{0}(b)$.

Observe that the condition $F_{0}(\hat{y}, \hat{z})<c_{0}(a)$ implies that $c_{0}(a)>F_{0}^{*}$ and, similarly, the condition $F_{0}(\tilde{y}, \tilde{z})<c_{0}(b)$ implies that $c_{0}(b)>F_{0}^{*}$.

A sufficient condition for the monotonicity of $F_{0}(\cdot, z)$ in Condition 2.3(a)(ii) is that, for each $z \in \mathcal{E} \backslash\{a\}$, there exists some $y_{z}>a$ such that $c_{1}(\cdot, z)$ is differentiable in the interval $\left(a, y_{z}\right)$ and

$$
\frac{-\partial c_{1}(y, z) / \partial y+g_{0}^{\prime}(y)}{\zeta^{\prime}(y)}>F_{0}(y, z)
$$

A similar sufficient condition for the monotonicity of $F_{0}$ in Condition 2.3(b)(ii) can be formulated.

Inequality (2.11) has an economic interpretation. For simplicity, assume that $(a, b) \subseteq$ $(0, \infty)$. The function $F_{0}$ on $\mathcal{R}$ represents the long-term average cost $(2.8)$ for each $(y, z)$ ordering policy as the ratio of the expected cost $c_{1}(y, z)+g_{0}(z)-g_{0}(y)$ over a cycle to the expected cycle length $\zeta(z)-\zeta(y)$ for the $(y, z)$ ordering policy.

Observe that $\zeta$ is a strictly increasing function, so the denominator of (2.11) is positive. Thus, using the definition of $F_{0}$ in (2.9), inequality (2.11) can equivalently be written as

$$
\frac{\partial\left[c_{1}(y, z)+g_{0}(z)-g_{0}(y)\right] / \partial y}{c_{1}(y, z)+g_{0}(z)-g_{0}(y)}<\frac{\partial[\zeta(z)-\zeta(y)] / \partial y}{\zeta(z)-\zeta(y)},
$$


and multiplying by $z$ on both sides preserves the inequality. Thus, this sufficient condition says that, for each $y \in \mathscr{E}$, there exists some ( $y$-dependent) neighborhood of $a$ on which the expected cost over a cycle is less elastic than the expected cycle length (with respect to variations of $z$ ). The similar analysis for the boundary $b$ using Condition 2.3(b) results in the reverse inequality and more elasticity.

The key result of this section is the existence of a minimizing pair $\left(y_{0}^{*}, z_{0}^{*}\right)$. This improves the result in Proposition 3.5 of Helmes et al. (2017); these relaxed conditions differ significantly enough that it is necessary to provide a careful proof. The proof is given in Appendix A.

Theorem 2.1. Assume that Conditions $2.1,2.2$, and 2.3 hold. Then there exists a pair $\left(y_{0}^{*}, z_{0}^{*}\right) \in$ $\mathcal{R}$ such that

$$
F_{0}\left(y_{0}^{*}, z_{0}^{*}\right)=F_{0}^{*}=\inf \left\{F_{0}(y, z):(y, z) \in \overline{\mathcal{R}}\right\} .
$$

An immediate corollary is that, when the optimization is restricted to the class of $(y, z)$ ordering policies, the minimal cost is achieved by the $\left(y_{0}^{*}, z_{0}^{*}\right)$ ordering policy and has an optimal value of $F_{0}^{*}$.

Corollary 2.1. Assume that Conditions 2.1, 2.2, and 2.3 hold, and let $\left(y_{0}^{*}, z_{0}^{*}\right)$ denote a minimizing pair for the function $F$. Then the ordering policy $\left(\tau^{*}, Y^{*}\right)$ defined using $\left(y_{0}^{*}, z_{0}^{*}\right)$ in (2.6) is optimal in the class of all $(s, S)$ ordering policies with corresponding optimal value of $F_{0}^{*}=F_{0}\left(y_{0}^{*}, z_{0}^{*}\right)=J_{0}\left(\tau^{*}, Y^{*}\right)$.

\section{Expected occupation and ordering measures}

To establish general optimality of the $\left(y_{0}^{*}, z_{0}^{*}\right)$ policy, we apply weak convergence arguments with average expected occupation and average expected ordering measures, which we now define.

For $(\tau, Y) \in \mathcal{A}$, let $X$ denote the resulting inventory level process satisfying (1.2). For each $t>0$, define the average expected occupation measure $\mu_{0, t}$ on $\mathscr{E}$ and the average expected ordering measure $\mu_{1, t}$ on $\overline{\mathcal{R}}$ by

$$
\begin{gathered}
\mu_{0, t}\left(\Gamma_{0}\right)=\frac{1}{t} \mathbb{E}\left[\int_{0}^{t} \mathbf{1}_{\Gamma_{0}}(X(s)) \mathrm{d} s\right], \quad \Gamma_{0} \in \mathcal{B}(\mathcal{E}), \\
\mu_{1, t}\left(\Gamma_{1}\right)=\frac{1}{t} \mathbb{E}\left[\sum_{k=1}^{\infty} \mathbf{1}_{\left\{\tau_{k} \leq t\right\}} \mathbf{1}_{\Gamma_{1}}\left(X\left(\tau_{k}-\right), X\left(\tau_{k}\right)\right)\right], \quad \Gamma_{1} \in \mathcal{B}(\overline{\mathcal{R}}) .
\end{gathered}
$$

If $a$ is a reflecting boundary, define the average expected local time measure $\mu_{2, t}$ for each $t>0$ to place a point mass on $\{a\}$ given by

$$
\mu_{2, t}(\{a\})=\frac{1}{t} \mathbb{E}\left[L_{a}(t)\right]
$$

in which $L_{a}$ denotes the local time of $X$ at $a$.

Remark 3.1. For each $t>0$, the average expected occupation measure $\mu_{0, t}$ is a probability measure on $\mathcal{E}$. In addition, for each $(\tau, Y) \in \mathcal{A}$ with $J_{0}(\tau, Y)<\infty, \mu_{1, t}$ has finite mass for each $t$ and $\lim \sup _{t \rightarrow \infty} \mu_{1, t}(\overline{\mathcal{R}}) \leq J_{0}(\tau, Y) / k_{1}$. Finally, observe that, when $a$ is a sticky boundary, $\mu_{0, t}$ places a point mass at $a$ for those policies $(\tau, Y)$ that allow the process $X$ to stick at $a$ with positive probability. 
For general policies $(\tau, Y)$, we are interested in the relative compactness of the collection of $\mu_{0, t}$ measures and the associated convergence (or not) of the functionals with integrand $c_{0}$. The first proposition below shows that, for any sequence $\left\{t_{i}\right\}$ going to $\infty,\left\{\mu_{0, t_{i}}\right\}$ is tight for policies $(\tau, Y)$ having finite costs. To understand the second proposition, consider the $(y, z)$ ordering policy defined by (2.6) for $(y, z) \in \mathcal{R}$. The $L^{1}$ convergence in (2.7) implies the remarkable result that, for $(y, z)$ policies,

$$
\lim _{t \rightarrow \infty} \int_{\mathcal{E}} c_{0}(x) \mu_{0, t}(\mathrm{~d} x)=\int_{\mathcal{E}} c_{0}(x) \mu_{0}(\mathrm{~d} x),
$$

even when $c_{0}$ is unbounded, in which $\mu_{0}$ is the unique stationary measure on $\mathcal{E}$. Proposition 3.2 demonstrates that (3.2) may not hold for general policies, but rather an inequality relation holds.

Proposition 3.1. Assume that Conditions 2.1 and 2.2 hold. For $(\tau, Y) \in \mathcal{A}$, let $X$ denote the resulting inventory process satisfying (1.2). Let $\left\{t_{i}: i \in \mathbb{N}\right\}$ be a sequence such that $\lim _{i \rightarrow \infty} t_{i}=\infty$ and, for each $i$, define $\mu_{0, t_{i}}$ by (3.1). If $\left\{\mu_{0, t_{i}}: i \in \mathbb{N}\right\}$ is not tight then $J_{0}(\tau, Y)=\infty$.

Proof. If $a$ and $b$ are both finite, then each $\mu_{0, t}$ has its support in $\overline{\mathcal{E}}=[a, b]$ regardless of the types of boundary point. The collection $\left\{\mu_{0, t}: t \geq 1\right\}$ is therefore tight. Thus, for this collection not to be tight, either $a=-\infty$ or $b=\infty$.

Suppose that $\left\{\mu_{0, t_{i}}\right\}$ is not tight. By Condition 2.2(a), $c_{0}(x) \rightarrow \infty$ as $x \rightarrow a$ or as $x \rightarrow b$. The lack of tightness of $\left\{\mu_{0, t_{i}}\right\}$ means that there exists some $\varepsilon>0$ such that, for all compact sets $K$ and $T>0$, there exists $t_{i} \geq T$ for which $\mu_{0, t_{i}}\left(K^{c}\right) \geq \varepsilon$. Arbitrarily select $M<\infty$ and set $K=\left\{x \in \bar{\varepsilon}: c_{0}(x) \leq M / \varepsilon\right\}$, noting that $K$ is compact. Then there exists a subsequence $\left\{t_{i_{j}}: i_{j} \in \mathbb{N}\right\}$ for which $\mu_{0, t_{i j}}\left(K^{c}\right) \geq \varepsilon$. Therefore, for each $i_{j}$,

$t_{i_{j}}^{-1} \mathbb{E}\left[\int_{0}^{t_{i_{j}}} c_{0}(X(s)) \mathrm{d} s\right]=\int_{\mathcal{E}} c_{0}(x) \mu_{0, t_{i j}}(\mathrm{~d} x) \geq \int_{K^{c}} c_{0}(x) \mu_{0, t_{i j}}(\mathrm{~d} x) \geq \frac{M}{\varepsilon} \mu_{0, t_{i_{j}}}\left(K^{c}\right) \geq M$.

Thus, $\lim \sup _{t \rightarrow \infty} t^{-1} \mathbb{E}\left[\int_{0}^{t} c_{0}(X(s)) \mathrm{d} s\right] \geq M$. It therefore follows that $J_{0}(\tau, Y)=\infty$ since $M$ is arbitrary.

The above proof shows that the tightness of $\left\{\mu_{0, t}\right\}$ follows from the finiteness of the longterm average holding costs, $\lim _{t \rightarrow \infty}(1 / t) \mathbb{E}\left[\int_{0}^{t} c_{0}(X(s)) \mathrm{d} s\right]$, regardless of the limiting behavior of the average expected ordering costs.

As a consequence of Proposition 3.1, when either $a=-\infty$ or $b=\infty$, we need to only consider those ordering policies $(\tau, Y)$ for which the collection $\left\{\mu_{0, t}: t \geq 0\right\}$ is tight. We denote a generic weak limit as $t \rightarrow \infty$ by $\mu_{0}$. Observe that the support of $\mu_{0}$ might be $\bar{\varepsilon}$. The continuity assumptions on $c_{0}$ at the boundaries in Condition 2.2 are required due to the limiting measures possibly placing mass there.

Proposition 3.2. Assume that Conditions 2.1 and 2.2 hold. Let $(\tau, Y) \in \mathcal{A}$ with $J_{0}(\tau, Y)<\infty$, let $X$ satisfy (1.2), and define $\mu_{0, t}$ by (3.1) for each $t>0$. Then, for each $\mu_{0}$ attained as a weak limit of some sequence $\left\{\mu_{0, t_{j}}\right\}$ as $t_{j} \rightarrow \infty$,

$$
\int_{\bar{\varepsilon}} c_{0}(x) \mu_{0}(\mathrm{~d} x) \leq J_{0}(\tau, Y)<\infty .
$$

Proof. Let $\mu_{0},\left\{t_{j}\right\}$, and $\left\{\mu_{0, t_{j}}\right\}$ be as in the statement of the proposition. Since $\left\{\mu_{0, t_{j}}\right\}, \mu_{0} \in$ $\mathcal{P}(\overline{\mathcal{E}})$, the Skorokhod representation theorem implies there exist random variables $\left\{\boldsymbol{\Xi}_{j}\right\}$ and $\Xi$, defined on a common probability space $(\tilde{\Omega}, \tilde{\mathcal{F}}, \tilde{\mathbb{P}})$, such that $\Xi$ has distribution $\mu_{0}, \Xi_{j}$ has 
distribution $\mu_{0, t_{j}}$ for each $j$, and $\Xi_{j} \rightarrow \Xi$ almost surely as $j \rightarrow \infty$. Since $c_{0}$ is bounded below, we may apply Fatou's lemma to obtain

$$
\begin{aligned}
\int_{\overline{\mathcal{E}}} c_{0}(x) \mu_{0}(\mathrm{~d} x) & =\tilde{\mathbb{E}}\left[c_{0}(\Xi)\right] \\
& \leq \liminf _{j \rightarrow \infty} \tilde{\mathbb{E}}\left[c_{0}\left(\Xi_{j}\right)\right] \\
& =\liminf _{j \rightarrow \infty} \int_{\overline{\bar{\varepsilon}}} c_{0}(x) \mu_{0, t_{j}}(\mathrm{~d} x) \\
& \leq J_{0}(\tau, Y) \\
& <\infty .
\end{aligned}
$$

We note that $c_{0}$ being infinite at a boundary implies that $\mu_{0}$ cannot assign any positive mass at this point. In particular, for models in which $a$ is a sticky boundary and $c_{0}(a)=\infty$, any policy which allows $X$ to stick at $a$ on a set of positive probability incurs an infinite average expected cost for each $t$ and, thus, has $J_{0}(\tau, Y)=\infty$. The condition $J_{0}(\tau, Y)<\infty$ therefore eliminates such $(\tau, Y)$ from consideration.

This inf-compactness of $c_{0}$ is central to the finiteness of $J_{0}(\tau, Y)$, implying that $\left\{\mu_{0, t}\right\}$ is tight as $t \rightarrow \infty$. A similar result follows in the case that $c_{1}$ is inf-compact as well. However, infcompactness fails for many natural ordering cost functions; in particular, the most commonly studied function $c_{1}(y, z)=k_{1}+k_{2}(z-y),(y, z) \in \mathcal{R}$, comprising fixed plus proportional costs is not inf-compact on an unbounded region of $\mathbb{R}^{2}$. Appendix $C$ contains an example which shows that even the requirement of a finite long-term average cost is insufficient to guarantee tightness of $\left\{\mu_{1, t}\right\}$ as $t \rightarrow \infty$. Fortunately, our method of solution does not rely on tightness of the average expected ordering measures.

\section{The auxiliary function $G_{0}$}

Our extension of the optimality of the $\left(y_{0}^{*}, z_{0}^{*}\right)$ policy to the class $\mathcal{A}$ requires the existence of an optimizing pair $\left(y_{0}^{*}, z_{0}^{*}\right) \in \mathcal{R}$ with $F_{0}\left(y_{0}^{*}, z_{0}^{*}\right)=F_{0}^{*}$, which we now impose on the models. Note that Condition 2.3 is a sufficient condition for such a pair to exist. Recall that Condition 2.2 requires continuity of $c_{0}$ at the boundary, even for finite, natural boundaries; $c_{0}$ may take value $\infty$ at the boundaries.

Define the auxiliary function $G_{0}$ on $\&$ by

$$
G_{0}=g_{0}-F_{0}^{*} \zeta
$$

and observe that $G_{0} \in C(\mathcal{E}) \cap C^{2}(\ell)$. Moreover, $G_{0}$ extends uniquely to $\bar{\varepsilon}$ due to the existence of $(\hat{y}, \hat{z})$ and $(\tilde{y}, \tilde{z})$ in Condition 2.3 or $c_{0}$ being infinite at the boundaries. This observation follows immediately when $a$ is attainable and when $b$ is an entrance boundary since $\zeta$ is finite in these cases. When $a$ or $b$ are natural boundaries, Lemma 2.1 combined with Condition 2.3 shows that

$$
\lim _{x \rightarrow a} G_{0}(x)=\lim _{x \rightarrow a}\left(g_{0}(x)-F_{0}^{*} \zeta(x)\right)=\lim _{x \rightarrow a}\left(\frac{g_{0}(x)}{\zeta(x)}-F_{0}^{*}\right) \zeta(x)=-\infty,
$$

and, similarly, $\lim _{x \rightarrow b} G_{0}(x)=\infty$.

Remark 4.1. The function $G_{0}$ differs from the function $G$ used in Helmes et al. (2017); $G_{0}$ is defined globally using (4.1), whereas $G$ agrees with $G_{0}$ on the set $\left[y_{0}^{*}, b\right]$, but is $C^{1}$-smoothly pasted with $c_{1}\left(\cdot, z_{0}^{*}\right)$ on $\left[a, y_{0}^{*}\right]$ (using the notation in this paper). The proof of optimality is significantly simplified since $G_{0}$ has a single expression. 
The function $G_{0}$ has the following interpretation. Let $y, z \in \mathcal{E}$. Then

$$
\begin{aligned}
c_{1}(y, z)+B G_{0}(y, z) & =c_{1}(y, z)+B g_{0}(y, z)-F_{0}^{*} B \zeta(y, z) \\
& =\left(\frac{c_{1}(y, z)+B g_{0}(y, z)}{B \zeta(y, z)}-F_{0}^{*}\right) B \zeta(y, z) \\
& =\left(F_{0}(y, z)-F_{0}^{*}\right) B \zeta(y, z) .
\end{aligned}
$$

Note that the relation $F_{0}^{*} \leq F_{0}(y, z)$ holds for all $(y, z) \in \mathcal{R}$. Thus, the function $c_{1}(y, z)+$ $B G_{0}(y, z)$ gives the increase in cost over a cycle incurred by using the $(y, z)$ ordering policy rather than an optimal ordering policy.

The function $G$ of Helmes et al. (2017) has the same interpretation for $y \geq y_{0}^{*}$. But, for $y<y_{0}^{*}$, it arises from a policy which places an immediate order from $y$ to $z_{0}^{*}$ and then follows the $\left(y_{0}^{*}, z_{0}^{*}\right)$ policy.

The function $G_{0}$ satisfies an important system of relations.

Proposition 4.1. Assume that Conditions 2.1, 2.2, and 2.3 hold, and let $G_{0}$ be as in (4.1). Then $G_{0}$ is a solution of the system

$$
\begin{gathered}
A f(x)+c_{0}(x)-F_{0}^{*}=0, \quad x \in \ell, \\
B f(y, z)+c_{1}(y, z) \geq 0, \quad(y, z) \in \overline{\mathcal{R}}, \\
f\left(x_{0}\right)=0, \\
B f\left(y_{0}^{*}, z_{0}^{*}\right)+c_{1}\left(y_{0}^{*}, z_{0}^{*}\right)=0 .
\end{gathered}
$$

Moreover, the first relation extends by continuity to $\bar{\varepsilon}$.

The proof is straightforward, so is left to the reader.

As a small digression to illuminate the definitions of $g_{0}$ and $\zeta$, the next proposition establishes an important transversality condition involving $G_{0}$ with the ensuing remark providing further clarification.

Proposition 4.2. Assume that Conditions 2.1, 2.2, and 2.3 hold. Let $x_{0} \in \ell$ be fixed. For $a \leq y<z<b$, let $(\tau, Y)$ be the $(y, z)$ ordering policy defined by (2.6) and let $X$ satisfy (1.2). Define the process $\tilde{M}$ by

$$
\tilde{M}(t):=\int_{0}^{t} \sigma(X(s)) G_{0}^{\prime}(X(s)) \mathrm{d} W(s), \quad t \geq 0 .
$$

Then there exists a localizing sequence $\left\{\beta_{n}: n \in \mathbb{N}\right\}$ of stopping times such that, for each $n, \tilde{M}\left(\cdot \wedge \beta_{n}\right)$ is a martingale and the following transversality condition holds:

$$
\lim _{t \rightarrow \infty} \lim _{n \rightarrow \infty} \frac{1}{t} \mathbb{E}\left[G_{0}\left(X\left(t \wedge \beta_{n}\right)\right)\right]=0 .
$$

In addition, defining $\mu_{0}$ to be the stationary measure and $\mu_{1}$ to place point mass $\kappa=1 / B \zeta(y, z)$ (the long-run frequency of orders) on $\{(y, z)\}$, we have

$$
\int_{\mathcal{E}} A G_{0}(x) \mu_{0}(\mathrm{~d} x)+\int_{\mathcal{R}} B G_{0}(y, z) \mu_{1}(\mathrm{~d} y \times \mathrm{d} z)=0 .
$$


Proof. Let $(y, z),(\tau, Y)$, and $X$ be as in the statement of the proposition. Let $\left\{b_{n}: n \in\right.$ $\mathbb{N}\} \subset \ell$ be a strictly increasing sequence such that $x_{0} \vee z \leq b_{1}$ and $\lim _{n \rightarrow \infty} b_{n}=b$, and define the sequence of localizing times $\left\{\beta_{n}: n \in \mathbb{N}\right\}$ by $\beta_{n}=\inf \left\{t \geq 0: X(t)=b_{n}\right\}$. Using Itô's formula, it follows that, for each $n$,

$$
\begin{aligned}
G_{0}\left(X\left(t \wedge \beta_{n}\right)\right)= & G_{0}\left(x_{0}\right)+\int_{0}^{t \wedge \beta_{n}} A G_{0}(X(s)) \mathrm{d} s+\int_{0}^{t \wedge \beta_{n}} \sigma(X(s)) G_{0}^{\prime}(X(s)) \mathrm{d} W(s) \\
& +\sum_{k=1}^{\infty} \mathbf{1}_{\left\{\tau_{k} \leq t \wedge \beta_{n}\right\}} B G_{0}\left(X\left(\tau_{k}-\right), X\left(\tau_{k}\right)\right) ;
\end{aligned}
$$

note that no local time term is included in this identity when $a$ is a reflecting boundary even when $y=a$ since an order is placed the first time $X$ hits $a$, so no local time is accrued. Taking expectations and using the fact that $A G_{0}=F_{0}^{*}-c_{0}$, we then obtain

$$
\begin{aligned}
\mathbb{E}\left[G_{0}\left(X\left(t \wedge \beta_{n}\right)\right)\right]= & G_{0}\left(x_{0}\right)+F_{0}^{*} \mathbb{E}\left[t \wedge \beta_{n}\right]-\mathbb{E}\left[\int_{0}^{t \wedge \beta_{n}} c_{0}(X(s)) \mathrm{d} s\right] \\
& +\mathbb{E}\left[\sum_{k=1}^{\infty} \mathbf{1}_{\left\{\tau_{k} \leq t \wedge \beta_{n}\right\}} B G_{0}\left(X\left(\tau_{k}-\right), X\left(\tau_{k}\right)\right)\right] .
\end{aligned}
$$

Since $b$ is inaccessible from the interior and $z<b$, it follows that $\beta_{n} \rightarrow \infty$ (a.s.) as $n \rightarrow \infty$. Applying the monotone and dominated convergence theorems, we have

$$
\begin{aligned}
\lim _{n \rightarrow \infty} \mathbb{E}\left[G_{0}\left(X\left(t \wedge \beta_{n}\right)\right)\right] & G_{0}\left(x_{0}\right)+F_{0}^{*} t-\mathbb{E}\left[\int_{0}^{t} c_{0}(X(s)) \mathrm{d} s+\sum_{k=1}^{\infty} \mathbf{1}_{\left\{\tau_{k} \leq t\right\}} B G_{0}\left(X\left(\tau_{k}-\right), X\left(\tau_{k}\right)\right)\right] \\
= & G_{0}\left(x_{0}\right)+F_{0}^{*} t-\mathbb{E}\left[\int_{0}^{t} c_{0}(X(s)) \mathrm{d} s\right]+B G_{0}(y, z) \mathbb{E}\left[\sum_{k=1}^{\infty} \mathbf{1}_{\left\{\tau_{k} \leq t\right\}}\right] \\
& +\left(B G_{0}\left(x_{0}, z\right)-B G_{0}(y, z)\right) \mathbb{E}\left[\mathbf{1}_{\left\{\tau_{1}=0\right\}}\right] .
\end{aligned}
$$

Divide by $t$ and let $t \rightarrow \infty$. Note that $\lim _{t \rightarrow \infty}(1 / t) \mathbb{E}\left[\sum_{k=1}^{\infty} \mathbf{1}_{\left\{\tau_{k} \leq t\right\}}\right]=1 / B \zeta(y, z)$ and the $L^{1}$ convergence in (2.7) gives $\lim _{t \rightarrow \infty}(1 / t) \mathbb{E}\left[\int_{0}^{t} c_{0}(X(s)) \mathrm{d} s\right]=B g_{0}(y, z) / B \zeta(y, z)$. Therefore,

$$
\lim _{t \rightarrow \infty} \lim _{n \rightarrow \infty} \frac{1}{t} \mathbb{E}\left[G_{0}\left(X\left(t \wedge \beta_{n}\right)\right)\right]=F_{0}^{*}-\frac{B g_{0}(y, z)}{B \zeta(y, z)}+\frac{B g_{0}(y, z)-F_{0}^{*} B \zeta(y, z)}{B \zeta(y, z)}=0 .
$$

Using the definitions of $\mu_{0, t}$ and $\mu_{1, t}$ in (3.1), this argument establishes that

$$
\lim _{t \rightarrow \infty}\left[\int_{\mathcal{E}} A G_{0}(x) \mu_{0, t}(\mathrm{~d} x)+\int_{\mathcal{R}} B G_{0}(y, z) \mu_{1, t}(\mathrm{~d} y \times \mathrm{d} z)\right]=0 .
$$

Since $A G_{0}=F_{0}^{*}-c_{0}$, by (3.2) and the definition of weak convergence,

$$
\lim _{t \rightarrow \infty} \int_{\mathscr{E}} A G_{0}(x) \mu_{0, t}(\mathrm{~d} x)=\int_{\mathscr{E}} A G_{0}(x) \mu_{0}(\mathrm{~d} x) .
$$

Turning to the convergence involving $\mu_{1, t}$, the $(y, z)$ ordering policy places one order at time 0 when $x_{0}<y$, so $\mu_{1, t}$ has mass on $\left(x_{0}, z\right)$, but thereafter orders are placed only when 
$X(t-)=y$, so all further mass of $\mu_{1, t}$ is on $\{(y, z)\}$. When $x_{0} \geq y, \mu_{1, t}$ only has mass on $\{(y, z)\}$. This implies that $\mu_{1, t} \Rightarrow \mu_{1}$ as $t \rightarrow \infty$, which in turn implies that

$$
\lim _{t \rightarrow \infty} \int_{\mathcal{R}} B G_{0}(y, z) \mu_{1, t}(\mathrm{~d} y \times \mathrm{d} z)=\int_{\mathcal{R}} B G_{0}(y, z) \mu_{1}(\mathrm{~d} y \times \mathrm{d} z) .
$$

Combining (4.7), (4.8), and (4.9) establishes (4.5).

Remark 4.2. By definition, the functions $g_{0}$ and $\zeta$ are solutions of $A f=-c_{0}$ and $A f=-1$, respectively, for which $g_{0}\left(x_{0}\right)=0=\zeta\left(x_{0}\right)$. The choice of the function $G_{0}=g_{0}-F_{0}^{*} \zeta$ is the only $C^{2}$ solution of the inhomogeneous equation $A f=F_{0}^{*}-c_{0}$ with $f\left(x_{0}\right)=0$ for which the transversality condition (4.4) holds. To see this, recall that the scale function $S$ and the constant function are two linearly independent solutions to the homogeneous equation $A f=0$. Define the function $\hat{S}(x)=S(x)-S\left(x_{0}\right)$ for $x \in \mathcal{E}$, so that $\hat{S}$ is a solution of $A f=0$ with $\hat{S}\left(x_{0}\right)=0$. Following the previous proof to (4.6), using $\hat{S}$ yields

$$
\begin{aligned}
\mathbb{E}\left[\hat{S}\left(X\left(t \wedge \beta_{n}\right)\right)\right] & =\mathbb{E}\left[\sum_{k=1}^{\infty} \mathbf{1}_{\left\{\tau_{k} \leq t \wedge \beta_{n}\right\}} B \hat{S}\left(X\left(\tau_{k}-\right), X\left(\tau_{k}\right)\right)\right] \\
& =B \hat{S}(y, z) \mathbb{E}\left[\sum_{k=1}^{\infty} \mathbf{1}_{\left\{\tau_{k} \leq t \wedge \beta_{n}\right\}}\right]+\left[B \hat{S}\left(x_{0}, z\right)-B \hat{S}(y, z)\right] \mathbb{E}\left[\mathbf{1}_{\left\{\tau_{k}=0\right\}}\right] .
\end{aligned}
$$

Thus, letting $n \rightarrow \infty$, dividing by $t$, and letting $t \rightarrow \infty$ yields

$$
\lim _{t \rightarrow \infty} \lim _{n \rightarrow \infty} \frac{1}{t} \mathbb{E}\left[\hat{S}\left(X\left(t \wedge \beta_{n}\right)\right)\right]=\frac{B \hat{S}(y, z)}{B \zeta(y, z)}=\frac{S(z)-S(y)}{\zeta(z)-\zeta(y)}>0 .
$$

Observe that, for $K \in \mathbb{R}, \hat{G}_{0}=G_{0}+K \hat{S}$ is the general solution to the inhomogeneous equation $A f=F_{0}^{*}-c_{0}$ with $f\left(x_{0}\right)=0$. As a result of Proposition 4.2 and (4.10), we have

$$
\lim _{t \rightarrow \infty} \lim _{n \rightarrow \infty} \frac{1}{t} \mathbb{E}\left[\hat{G}_{0}\left(X\left(t \wedge \beta_{n}\right)\right)\right]=\frac{K(S(z)-S(y))}{\zeta(z)-\zeta(y)},
$$

and the transversality condition (4.4) fails for any $\hat{G}_{0}$ with nonzero $K$.

\section{Policy class $\mathcal{A}_{0}$ and optimality}

For models having a reflecting boundary point $a$, we are able to prove optimality of the $\left(y_{0}^{*}, z_{0}^{*}\right)$ ordering policy within a slightly restricted class of admissible policies. (Note that there is no restriction on the class $\mathcal{A}$ when $a$ is not a reflecting boundary.)

Definition 5.1. For models in which $a$ is a reflecting boundary point, the class $\mathcal{A}_{0} \subset \mathcal{A}$ consists of those policies $(\tau, Y)$ for which the transversality condition

$$
\lim _{t \rightarrow \infty} t^{-1} \mathbb{E}\left[L_{a}(t)\right]=0
$$

holds; recall that $L_{a}$ denotes the local time process of $X$ at $a$.

Referring to Definition 3.11 of Helmes et al. (2017), restriction of the admissible class is also required in our previous paper. In particular, the class $\mathcal{A}_{1}$ in that paper requires (5.1) along with the existence of a localizing sequence $\left\{\beta_{n}\right\}$ such that the stopped processes (4.3) are martingales 
and, using limits inferior, the additional transversality condition (4.4) holds; the conditions use the function $G$ of that paper. This paper requires only the transversality condition (5.1) on the local time process.

For $(y, z)$ ordering policies, Proposition 4.2 shows that the corresponding limiting measures $\left(\mu_{0}, \mu_{1}\right)$ of the pairs $\left\{\left(\mu_{0, t}, \mu_{1, t}\right): t>0\right\}$ satisfy (4.5). However, for a general ordering policy $(\tau, Y) \in \mathcal{A}_{0}$, the measures $\left\{\mu_{1, t}\right\}$ may not have weak limits as $t \rightarrow \infty$. We establish the limiting adjoint relation (5.2) below for a class of test functions $\mathscr{D}$, defined below, which is used to show that $J_{0}(\tau, Y) \geq F_{0}^{*}$ for any $(\tau, Y) \in \mathcal{A}_{0}$.

Recall that Proposition 3.1 demonstrates that the measures $\left\{\mu_{0, t}\right\}$ are tight (as $t \rightarrow \infty$ ) whenever $J_{0}(\tau, Y)<\infty$. It follows that any weak limit $\mu_{0}$ will have support in $\bar{\varepsilon}$. However, when $c_{0}(a)=\infty$ or $c_{0}(b)=\infty, J_{0}(\tau, Y)<\infty$ implies that each limiting measure $\mu_{0}$ places no mass on the corresponding boundary.

We begin by identifying a particular class of test functions that plays a key role in the analysis.

Definition 5.2. A function $f$ is in $\mathscr{D}$ provided it satisfies

(a) $f \in C(\overline{\mathcal{E}}) \cap C^{2}(\ell)$ and there exists $L_{f}<\infty$ such that

(i) $|f| \leq L_{f}$;

(ii) $\left(\sigma f^{\prime}\right)^{2} \leq L_{f}\left(1+c_{0}\right)$;

(iii) $|A f| \leq L_{f}$;

(b) (i) for all models, at each boundary where $c_{0}$ is finite, $A f$ extends continuously to the boundary with a finite value;

(ii) when $a$ is a reflecting boundary, $\left|f^{\prime}(a)\right|<\infty$; and

(iii) when $a$ is a sticky boundary and $c_{0}(a)<\infty, \sigma f^{\prime}$ extends continuously at $a$ to a finite value.

As pointed out at the end of Section 3, any policy $(\tau, Y)$ which allows $X$ to stick at $a$ with positive probability when $c_{0}(a)=\infty$ has $J_{0}(\tau, Y)=\infty$, so is clearly not optimal. Thus, the stickiness of $a$ is of concern only when $c_{0}(a)<\infty$, in which case Definition 5.2(b)(i) and (b)(iii) are required for our analysis.

Proposition 5.1. Assume that Conditions 2.1 and 2.2 hold. Let $(\tau, Y) \in \mathcal{A}_{0}$ with $J_{0}(\tau, Y)<$ $\infty$, and let $X$ satisfy (1.2). For $t>0$, define $\left(\mu_{0, t}, \mu_{1, t}\right)$ by (3.1) and let $\mu_{0}$ be such that $\mu_{0, t_{j}} \Rightarrow \mu_{0}$ as $j \rightarrow \infty$ for some sequence $\left\{t_{j}: j \in \mathbb{N}\right\}$ with $\lim _{j \rightarrow \infty} t_{j}=\infty$. Then the limiting adjoint relation

$$
\int_{\overline{\mathcal{E}}} A f(x) \mu_{0}(\mathrm{~d} x)+\lim _{j \rightarrow \infty} \int_{\overline{\mathcal{R}}} B f(y, z) \mu_{1, t_{j}}(\mathrm{~d} y \times \mathrm{d} z)=0 \text { for all } f \in \mathscr{D}
$$

holds.

Remark 5.1. For policies $(\tau, Y)$ with $J_{0}(\tau, Y)<\infty$, Proposition 3.1 only establishes tightness of the average expected occupation measures $\left\{\mu_{0, t}\right\}$, but not tightness of the average expected ordering measures $\left\{\mu_{1, t}\right\}$. Ordering policies for which the collection $\left\{\mu_{1, t}\right\}$ is tight as $t \rightarrow \infty$ will have weak limits. For any pair $\left(\mu_{0}, \mu_{1}\right)$ with $\left(\mu_{0, t_{j}}, \mu_{1, t_{j}}\right) \Rightarrow\left(\mu_{0}, \mu_{1}\right)$ as $j \rightarrow \infty,(5.2)$ is more simply expressed as

$$
\int_{\overline{\mathcal{E}}} A f(x) \mu_{0}(\mathrm{~d} x)+\int_{\overline{\mathcal{R}}} B f(y, z) \mu_{1}(\mathrm{~d} y \times \mathrm{d} z)=0 \quad \text { for all } f \in \mathscr{D} .
$$


Proof of Proposition 5.1. Let ( $\tau, Y), X,\left\{t_{j}\right\},\left\{\left(\mu_{0, t_{j}}, \mu_{1, t_{j}}\right)\right\}$, and $\mu_{0}$ be as in the statement of the proposition. The following analysis considers the case of $a$ being a reflecting boundary; when $a$ is not reflecting, the local time term is omitted. For each $f \in \mathscr{D}$ and $j \in \mathbb{N}$, Itô's formula yields

$$
\begin{aligned}
f\left(X\left(t_{j}\right)\right)= & f\left(x_{0}\right)+\int_{0}^{t_{j}} A f(X(s)) \mathrm{d} s+\sum_{k=1}^{\infty} \mathbf{1}_{\left\{\tau_{k} \leq t_{j}\right\}} B f\left(X\left(\tau_{k}-\right), X\left(\tau_{k}\right)\right) \\
& +\int_{0}^{t_{j}} \sigma(X(s)) f^{\prime}(X(s)) \mathrm{d} W(s)+f^{\prime}(a) L_{a}\left(t_{j}\right) .
\end{aligned}
$$

Observe that, for each $t_{j}$,

$$
\mathbb{E}\left[\int_{0}^{t_{j}}\left(\sigma(X(s)) f^{\prime}(X(s))\right)^{2} \mathrm{~d} s\right] \leq 2 \mathbb{E}\left[\int_{0}^{t_{j}} L_{f}\left[1+c_{0}(X(s))\right] \mathrm{d} s\right]<\infty,
$$

so $\sigma f^{\prime} \in L^{2}\left(\Omega \times\left[0, t_{j}\right]\right)$ and the stochastic integral has mean 0 . Thus, taking expectations and dividing by $t_{j}$ yields

$$
\begin{aligned}
\frac{1}{t_{j}} \mathbb{E}_{x_{0}}\left[f\left(X\left(t_{j}\right)\right)\right]= & \frac{f\left(x_{0}\right)}{t_{j}}+\int_{\mathcal{E}} A f(x) \mu_{0, t_{j}}(\mathrm{~d} x)+\int_{\overline{\mathcal{R}}} B f(y, z) \mu_{1, t_{j}}(\mathrm{~d} y \times \mathrm{d} z) \\
& +f^{\prime}(a) \frac{\mathbb{E}\left[L_{a}\left(t_{j}\right)\right]}{t_{j}} .
\end{aligned}
$$

Observe carefully that $\overline{\mathcal{E}}$ differs from $\mathcal{E}$ only when at least one of the boundaries is finite and natural. Moreover, for each $t_{j}, \mu_{0, t_{j}}$ places no mass on these boundaries, so the integration may also be viewed as being over $\overline{\mathcal{E}}$ and this is required when we pass to the limit as $t_{j} \rightarrow \infty$ since the limiting measure $\mu_{0}$ may put positive mass on such a boundary point.

Since, by the definition of $\mathcal{D}$, the function $f \in C(\overline{\mathcal{E}}) \cap C^{2}(\ell)$ is bounded, implying that $B f$ is also bounded and continuous. In addition, $A f$ is bounded and continuous, and extends continuously to a finite value at $a$ when $a$ is a finite, natural boundary, and $f^{\prime}(a)$ is finite. Upon letting $j \rightarrow \infty$ we obtain

$$
\int_{\bar{\varepsilon}} A f(x) \mu_{0}(\mathrm{~d} x)+\lim _{j \rightarrow \infty} \int_{\overline{\mathcal{R}}} B f(y, z) \mu_{1, t_{j}}(\mathrm{~d} y \times \mathrm{d} z)=0 ;
$$

the existence of $\lim _{j \rightarrow \infty} \int B f \mathrm{~d} \mu_{1, t_{j}}$ follows from the limits existing for the other terms in the previous equation. Note, in particular, that $\mu_{0}$ places no mass on boundaries where $c_{0}$ is infinite, so the weak convergence argument does not require $A f$ to extend continuously at such boundaries (cf. Definition 5.2(b)(i)). When $a$ is a sticky boundary, however, the extensions in Definition 5.2(b)(i) and (b)(iii) are required to apply Itô's formula.

Corollary 5.1. Assume that Conditions 2.1, 2.2, and 2.3 hold. Suppose that $G_{0} \in \mathcal{D}$. Then, for every $(\tau, Y) \in \mathcal{A}_{0}, J_{0}(\tau, Y) \geq F_{0}^{*}$ and, hence, the $\left(y_{0}^{*}, z_{0}^{*}\right)$ ordering policy is optimal in the class $\mathcal{A}_{0}$.

Proof. Let $(\tau, Y) \in \mathcal{A}_{0}, X$ satisfy (1.2), and let $\left\{t_{j}: j \in \mathbb{N}\right\}$ be a sequence such that

$$
J_{0}(\tau, Y)=\lim _{j \rightarrow \infty} \frac{1}{t_{j}} \mathbb{E}\left[\int_{0}^{t_{j}} c_{0}(X(s)) \mathrm{d} s+\sum_{k=1}^{\infty} \mathbf{1}_{\left\{\tau_{k} \leq t_{j}\right\}} c_{1}\left(X\left(\tau_{k}-\right), X\left(\tau_{k}\right)\right)\right] .
$$


By considering a subsequence, if necessary, let $\mu_{0, t_{j}} \Rightarrow \mu_{0}$ for some $\mu_{0} \in \mathcal{P}(\overline{\mathcal{E}})$. The combination of Propositions 4.1 and 5.1 immediately implies that

$$
\begin{aligned}
J_{0}(\tau, Y)= & \lim _{j \rightarrow \infty}\left(\int_{\overline{\mathcal{E}}} c_{0}(x) \mu_{0, t_{j}}(\mathrm{~d} x)+\int_{\overline{\mathcal{R}}} c_{1}(y, z) \mu_{1, t_{j}}(\mathrm{~d} y \times \mathrm{d} z)\right) \\
= & \lim _{j \rightarrow \infty}\left(\int_{\overline{\mathcal{E}}}\left(A G_{0}(x)+c_{0}(x)\right) \mu_{0, t_{j}}(\mathrm{~d} x)\right. \\
& \left.\quad \quad+\int_{\overline{\mathcal{R}}}\left(B G_{0}(y, z)+c_{1}(y, z)\right) \mu_{1, t_{j}}(\mathrm{~d} y \times \mathrm{d} z)\right) \\
\geq & \int_{\overline{\mathcal{E}}} F_{0}^{*} \mu_{0}(\mathrm{~d} x) \\
= & F_{0}^{*},
\end{aligned}
$$

and, thus, the $\left(y_{0}^{*}, z_{0}^{*}\right)$ ordering policy is optimal in the class $\mathcal{A}_{0}$.

In general, $G_{0} \notin \mathscr{D}$, so it is necessary to approximate $G_{0}$ by functions in $\mathscr{D}$ and pass to a limit. Interestingly, the following analysis works with the approximating functions $G_{n}$ defined in Lemma 5.1 without establishing an adjoint relation involving the function $G_{0}$.

Recall from (4.2) that, when $a$ is a natural boundary, $G_{0}(a):=\lim _{x \rightarrow a} G_{0}(x)=-\infty$, and, similarly, $G_{0}(b):=\lim _{x \rightarrow b} G_{0}(x)=\infty$ when $b$ is natural.

To proceed, we impose another set of conditions.

Condition 5.1. Let $G_{0}$ be as defined in (4.1).

(a) There exists some $L<\infty$ and some $y_{1}>$ a such that

(i) for models having $c_{0}(a)=\infty$,

$$
\frac{c_{0}(x)}{\left(1+\left|G_{0}(x)\right|\right)^{2}}+\frac{\left(\sigma(x) G_{0}^{\prime}(x)\right)^{2}}{\left(1+\left|G_{0}(x)\right|\right)^{3}} \leq L, \quad a<x<y_{1} ;
$$

(ii) for models in which $c_{0}(a)<\infty$, there is some $\varepsilon \in(0,1)$ such that

$$
\frac{\left(\sigma(x) G_{0}^{\prime}(x)\right)^{2}}{\left(1+\left|G_{0}(x)\right|\right)^{2+\varepsilon}} \leq L, \quad a \leq x<y_{1} .
$$

(b) There exists some $L<\infty$ and some $z_{1}<b$ such that

(i) for models having $c_{0}(b)=\infty$,

$$
\frac{c_{0}(x)}{\left(1+\left|G_{0}(x)\right|\right)^{2}}+\frac{\left(\sigma(x) G_{0}^{\prime}(x)\right)^{2}}{\left(1+\left|G_{0}(x)\right|\right)\left(1+c_{0}(x)\right)} \leq L, \quad z_{1}<x<b ;
$$

(ii) for models in which $c_{0}(b)<\infty$, there is some $\varepsilon \in(0,1)$ such that

$$
\frac{\left(\sigma(x) G_{0}^{\prime}(x)\right)^{2}}{\left(1+\left|G_{0}(x)\right|\right)^{2+\varepsilon}}+\frac{\left(\sigma(x) G_{0}^{\prime}(x)\right)^{2}}{\left(1+\left|G_{0}(x)\right|\right)\left(1+c_{0}(x)\right)} \leq L, \quad z_{1}<x \leq b .
$$

(c) (i) When $G_{0}(a)>-\infty$, or when a is a sticky boundary with $c_{0}(a)<\infty$, the limit $\lim _{x \rightarrow a} \sigma(x) G_{0}^{\prime}(x)$ exists and is finite;

(ii) when a is a reflecting boundary, $G_{0}^{\prime}(a)$ exists and is finite; and

(iii) when $G_{0}(b)<\infty, \lim _{x \rightarrow b} \sigma(x) G_{0}^{\prime}(x)$ exists and is finite. 
First note that the bound in Condition 5.1(b)(i) at the boundary $b$ is more restrictive than the similar bound in Condition 5.1(a)(i) at $a$ since

$$
\frac{\left(\sigma(x) G_{0}^{\prime}(x)\right)^{2}}{\left(1+\left|G_{0}(x)\right|\right)^{3}}=\frac{\left(\sigma(x) G_{0}^{\prime}(x)\right)^{2}}{\left(1+\left|G_{0}(x)\right|\right)\left(1+c_{0}(x)\right)} \frac{1+c_{0}(x)}{\left(1+\left|G_{0}(x)\right|\right)^{2}} \leq L(1+L) .
$$

The need for tighter restrictions at the boundary $b$ than at $a$ is not unexpected since there is no way to control the process from diffusing upwards, whereas ordering can prevent the process from diffusing towards $a$.

The reason for having two different conditions in Condition 5.1(a) and (b) based on whether $c_{0}$ at the boundary is finite or infinite is that any limiting pair of measures $\left(\mu_{0}, \mu_{1}\right)$ arising from an admissible policy $(\tau, Y)$ having finite $\operatorname{cost} J_{0}(\tau, Y)$ must place no $\mu_{0}$-mass at a boundary where $c_{0}$ is infinite. A weak limit $\mu_{0}$ may have positive mass at a boundary when $c_{0}$ is finite. Also, note the subtle assumption in Condition 5.1(a)(ii) and (b)(ii) that the bounds extend to the boundary, whereas there is no assumption needed at the boundary in Condition 5.1(a)(i) and (b)(i).

For models that satisfy Conditions 2.1, 2.2, and 2.3, Condition 5.1 places additional restrictions. In the online supplement (see Helmes et al. (2018)) we study a Feller branching diffusion inventory model; it illustrates the interplay between the cost rate function and the dynamics of the inventory process necessary for this condition to be satisfied.

Now define the elementary function $h$ on $\mathbb{R}$ which is central to the approximation of $G_{0}$ :

$$
h(x)= \begin{cases}-\frac{1}{8} x^{4}+\frac{3}{4} x^{2}+\frac{3}{8} & \text { for }|x| \leq 1 \\ |x| & \text { for }|x| \geq 1\end{cases}
$$

Note that $h \in C^{2}(\mathbb{R}), h>0$ and $h(x) \geq|x|$. For later reference, elementary calculations show that $h$ has the following properties on $\mathbb{R}$ :

- $h^{\prime}<0$ on $(-\infty, 0)$ and $h^{\prime}>0$ on $(0, \infty)$;

- $h^{\prime \prime}(x) \geq 0$ for all $x \in \mathbb{R}$ and $h^{\prime \prime}(x)=0$ for $|x| \geq 1$;

- $0 \leq h(x)-x h^{\prime}(x) \leq \frac{3}{8}$; and

- $h(x)+x h^{\prime}(x) \geq 0$.

In the next two lemmas, we define a sequence of functions $\left\{G_{n}: n \in \mathbb{N}\right\} \subset \mathscr{D}$ which approximate $G_{0}$, and examine the convergence of $A G_{n}$ and $B G_{n}$. The proofs are given in Appendix B.

Lemma 5.1. Assume that Conditions 2.1, 2.2, 2.3, and 5.1 hold with $G_{0}$ defined by (4.1). For each $n \in \mathbb{N}$, define the function $G_{n}$ by

$$
G_{n}=\frac{G_{0}}{1+h\left(G_{0}\right) / n} .
$$

Then $G_{n} \in \mathcal{D}$.

Lemma 5.2. Assume that Conditions 2.1, 2.2, 2.3, and 5.1 hold. Let $G_{n}$ be as defined in (5.4). Then

$$
\lim _{n \rightarrow \infty} A G_{n}(x)=A G_{0}(x) \text { for all } x \in \ell
$$

and

$$
\lim _{n \rightarrow \infty} B G_{n}(y, z)=B G_{0}(y, z) \quad \text { for all }(y, z) \in \overline{\mathcal{R}} .
$$

Moreover, at each boundary where $c_{0}$ is finite, $\lim _{n \rightarrow \infty} A G_{n} \geq A G_{0}$. 
The following proposition gives the first important result involving $A G_{n}$ and $c_{0}$.

Proposition 5.2. Assume that Conditions 2.1, 2.2, 2.3, and 5.1 hold. Let $(\tau, Y) \in \mathcal{A}_{0}$ with $J_{0}(\tau, Y)<\infty$, let $X$ satisfy (1.2), $\mu_{0, t}$ be defined by (3.1), and let $\mu_{0}$ be any weak limit of $\left\{\mu_{0, t}\right\}$ as $t \rightarrow \infty$. Define $G_{n}$ by (5.4). Then

$$
\liminf _{n \rightarrow \infty} \int_{\bar{\varepsilon}}\left(A G_{n}(x)+c_{0}(x)\right) \mu_{0}(\mathrm{~d} x) \geq \int_{\bar{\varepsilon}}\left(A G_{0}(x)+c_{0}(x)\right) \mu_{0}(\mathrm{~d} x) \geq F_{0}^{*} .
$$

Proof. Let ( $\tau, Y),\left\{\mu_{0, t}\right\}$, and $\mu_{0}$ be as in the statement of the proposition. Using (B.2) in Appendix $\mathrm{B}$, write $A G_{n}=A G_{n}^{(1)}+e_{n}^{(2)}+e_{n}^{(3)}$. Thus, for $x \in \ell$,

$$
\begin{aligned}
A G_{n}^{(1)}(x)+c_{0}(x)= & \left(-c_{0}(x)+F_{0}^{*}\right) \frac{\left[1+h\left(G_{0}(x)\right) / n-G_{0}(x) h^{\prime}\left(G_{0}(x)\right) / n\right]}{\left(1+h\left(G_{0}(x)\right) / n\right)^{2}}+c_{0}(x) \\
= & c_{0}(x) \frac{\left(h\left(G_{0}(x)\right)\right)^{2} / n^{2}+h\left(G_{0}(x)\right) / n+G_{0}(x) h^{\prime}\left(G_{0}(x)\right) / n}{\left(1+h\left(G_{0}(x)\right) / n\right)^{2}} \\
& +F_{0}^{*} \frac{\left[1+h\left(G_{0}(x)\right) / n-G_{0}(x) h^{\prime}\left(G_{0}(x)\right) / n\right]}{\left(1+h\left(G_{0}(x)\right) / n\right)^{2}} .
\end{aligned}
$$

As noted earlier, $h$ satisfies $h(x)+x h^{\prime}(x)>0$ and $0 \leq h(x)-x h^{\prime}(x) \leq \frac{3}{8}$ for all $x \in \mathbb{R}$. Thus, both terms in the rewritten expression for $A G_{n}^{(1)}(x)+c_{0}(x)$ are positive on $\ell$ and are nonnegative on $\overline{\mathcal{E}}$. Therefore, Fatou's lemma implies that

$$
\begin{aligned}
& \liminf _{n \rightarrow \infty} \int_{\overline{\mathcal{E}}}\left(A G_{n}^{(1)}(x)+c_{0}(x)\right) \mu_{0}(\mathrm{~d} x) \\
& \quad \geq \int_{\overline{\mathcal{E}}} \liminf _{n \rightarrow \infty}\left(A G_{n}^{(1)}(x)+c_{0}(x)\right) \mu_{0}(\mathrm{~d} x) \\
& \quad=\int_{\mathscr{E}} \liminf _{n \rightarrow \infty}\left(A G_{n}^{(1)}(x)+c_{0}(x)\right) \mu_{0}(\mathrm{~d} x)+\int_{\overline{\mathcal{E}} \backslash \mathcal{E}} \liminf _{n \rightarrow \infty}\left(A G_{n}^{(1)}(x)+c_{0}(x)\right) \mu_{0}(\mathrm{~d} x) .
\end{aligned}
$$

When $c_{0}(a)$ or $c_{0}(b)$ is infinite, the limiting measure $\mu_{0}$ does not place any mass at $a$ or $b$. Recall that $A G_{0}=F_{0}^{*}-c_{0}$ and $\left|G_{0}(x)\right|<\infty$ for all $x \in \ell$. For all $x \in \overline{\mathcal{E}}$ with $\left|G_{0}(x)\right|<\infty$, it follows that $\lim _{n \rightarrow \infty} A G_{n}^{(1)}(x)+c_{0}(x)=F_{0}^{*}$. Turning to the boundary $a$, when $\left|G_{0}(a)\right|=\infty$ and $c_{0}(a)<\infty$, then $A G_{n}^{(1)}(a)=0$ and, hence, Condition 2.3(a)(ii) implies that $A G_{n}^{(1)}(a)+$ $c_{0}(a)>F_{0}^{*}$. A similar observation applies to the right boundary $b$ when $\left|G_{0}(b)\right|=\infty$. Therefore, it follows that

$$
\liminf _{n \rightarrow \infty} \int_{\overline{\mathcal{E}}}\left(A G_{n}^{(1)}(x)+c_{0}(x)\right) \mu_{0}(\mathrm{~d} x) \geq \int_{\mathcal{E}} F_{0}^{*} \mu_{0}(\mathrm{~d} x)+\int_{\overline{\mathcal{E}} \backslash \mathcal{E}} F_{0}^{*} \mu_{0}(\mathrm{~d} x)=F_{0}^{*} .
$$

Now consider the term $e_{n}^{(2)}$. Recall that $h^{\prime \prime}(x)=0$ for all $x \in \mathbb{R}$ with $|x| \geq 1$. When $\left|G_{0}(a)\right|=\infty$, then $\lim _{x \rightarrow a} e_{n}^{(2)}(x)=0$; when $\left|G_{0}(a)\right|<\infty$, it follows from Condition 5.1(c) that $\lim _{x \rightarrow a} e_{n}^{(2)}(x)$ exists and is finite. A similar analysis applies to the right boundary $b$. From the definition of $e_{n}^{(2)}$, it therefore follows that $\left|e_{n}^{(2)}(x)\right| \leq K / n \leq K<\infty$ uniformly in $x$ and $n$. Thus, the bounded convergence theorem implies that

$$
\liminf _{n \rightarrow \infty} \int_{\bar{\varepsilon}} e_{n}^{(2)}(x) \mu_{0}(\mathrm{~d} x)=\int_{\bar{\varepsilon}} \liminf _{n \rightarrow \infty} e_{n}^{(2)}(x) \mu_{0}(\mathrm{~d} x)=0 .
$$

It remains to analyze the term $e_{n}^{(3)}(x)$. If $\left|G_{0}(a)\right|=\infty$ then, as argued in the proof of Lemma 5.2, we must have $G_{0}(a)=-\infty$. Consequently, there exists some $y_{1}>a$ such that 
$G_{0}(x)<0$ for all $a \leq x<y_{1}$. Thus, on $\left(a, y_{1}\right), h^{\prime}\left(G_{0}(x)\right)<0$ and $e_{n}^{(3)}(x) \geq 0$, and these relations extend by continuity to $a$. Applying Fatou's lemma, we have

$$
\liminf _{n \rightarrow \infty} \int_{\left[a, y_{1}\right)} e_{n}^{(3)}(x) \mu_{0}(\mathrm{~d} x) \geq \int_{\left[a, y_{1}\right)} \liminf _{n \rightarrow \infty} e_{n}^{(3)}(x) \mu_{0}(\mathrm{~d} x) \geq 0 .
$$

If $\left|G_{0}(a)\right|<\infty$ then Condition 5.1(c) implies that $\lim _{x \rightarrow a} \sigma(x) G_{0}^{\prime}(x)$ exists and is finite. This, together with the fact that $h^{\prime}(x)\left[1+h(x) / n-x h^{\prime}(x) / n\right]$ is uniformly bounded for all $x \in \mathbb{R}$ and $n \in \mathbb{N}$, implies that there exist a positive constant $K$ and some $y_{1}>a$ such that $\left|e_{n}^{(3)}(x)\right| \leq K / n \leq K$ for all $a \leq x<y_{1}$ and $n \in \mathbb{N}$. As a result, (5.7) still holds (with ' $\geq$ ' replaced by ' $=$ ') by virtue of the bounded convergence theorem. For all $x \in \overline{\mathcal{E}},\left|h^{\prime}\left(G_{0}(x)\right)\right|\left[1+h\left(G_{0}(x)\right) / n-G_{0}(x) h^{\prime}\left(G_{0}(x)\right) / n\right] \leq 2$, so, by Condition $5.1(\mathrm{~b})$, there exists some $z_{1}<b$ such that, for all $z_{1}<x \leq b$,

$$
\begin{aligned}
\left|e_{n}^{(3)}(x)\right| & \leq \frac{2\left(\sigma(x) G_{0}^{\prime}(x)\right)^{2}}{n\left(1+h\left(G_{0}(x)\right) / n\right)^{3}} \\
& =\frac{2\left(\sigma(x) G_{0}^{\prime}(x)\right)^{2}}{\left(n+\left|G_{0}(x)\right|\right)\left(1+c_{0}(x)\right)} \frac{1+c_{0}(x)}{\left(1+h\left(G_{0}(x)\right) / n\right)^{2}} \\
& \leq 2 L\left(1+c_{0}(x)\right) .
\end{aligned}
$$

The function $\left(1+c_{0}(x)\right)$ is integrable with respect to $\mu_{0}$ and, hence, the dominated convergence theorem implies that

$$
\lim _{n \rightarrow \infty} \int_{\left(z_{1}, b\right]} e_{n}^{(3)}(x) \mu_{0}(\mathrm{~d} x)=\int_{\left(z_{1}, b\right]} \lim _{n \rightarrow \infty} e_{n}^{(3)}(x) \mu_{0}(\mathrm{~d} x) .
$$

It is immediate from the definition of $e_{n}^{(3)}$ that $\lim _{n \rightarrow \infty} e_{n}^{(3)}(x)=0$ for $x \in\left(z_{1}, b\right)$; this limit also holds when $c_{0}(b)<\infty$ and $G_{0}(b)<\infty$. When $c_{0}(b)<\infty$ and $G_{0}(b)=\infty$, then continuity of $e_{n}^{(3)}$ along with Condition 5.1(b) implies that $e_{n}^{(3)}(b)=\lim _{x \rightarrow b} e_{n}^{(3)}(x)=0$. When $c_{0}(b)=\infty$, then $\mu_{0}$ places no mass at $b$. Thus, the dominated convergence theorem again implies that

$$
\lim _{n \rightarrow \infty} \int_{\left(z_{1}, b\right]} e_{n}^{(3)}(x) \mu_{0}(\mathrm{~d} x)=\int_{\left(z_{1}, b\right]} \lim _{n \rightarrow \infty} e_{n}^{(3)}(x) \mu_{0}(\mathrm{~d} x)=0 .
$$

Continuity implies that there exists some $K<\infty$ such that $\left(\sigma(x) G_{0}^{\prime}(x)\right)^{2}<K$ on the interval $\left[y_{1}, z_{1}\right]$ so $\left|e_{n}^{(3)}(x)\right|<2 K / n \leq 2 K$ and $\lim _{n \rightarrow \infty} e_{n}^{(3)}(x)=0$. Thus, the bounded convergence theorem also implies that

$$
\lim _{n \rightarrow \infty} \int_{\left[y_{1}, z_{1}\right]} e_{n}^{(3)}(x) \mu_{0}(\mathrm{~d} x)=\int_{\left[y_{1}, z_{1}\right]} \lim _{n \rightarrow \infty} e_{n}^{(3)}(x) \mu_{0}(\mathrm{~d} x)=0 .
$$

Combining (5.7)-(5.9) yields

$$
\liminf _{n \rightarrow \infty} \int_{\bar{\varepsilon}} e_{n}^{(3)}(x) \mu_{0}(\mathrm{~d} x) \geq 0 .
$$

In light of (B.2) in Appendix B, the result now follows from (5.5), (5.6), and (5.10).

We next establish a similar result involving $B G_{n}$ and $c_{1}$, though the lack of tightness of $\left\{\mu_{1, t}\right\}$ means that the result cannot be expressed in terms of a limiting measure $\mu_{1}$. 
Proposition 5.3. Assume that Conditions 2.1, 2.2, 2.3, and 5.1 hold. Let $(\tau, Y) \in \mathcal{A}_{0}$ with $J_{0}(\tau, Y)<\infty$, and let $X$ satisfy(1.2). Let $\left\{t_{j}: j \in \mathbb{N}\right\}$ be a sequence such that $\lim _{j \rightarrow \infty} t_{j}=\infty$ and

$$
J_{0}(\tau, Y)=\lim _{j \rightarrow \infty} \frac{1}{t_{j}} \mathbb{E}\left[\int_{0}^{t_{j}} c_{0}(X(s)) \mathrm{d} s+\sum_{k=1}^{\infty} \mathbf{1}_{\left\{\tau_{k} \leq t_{j}\right\}} c_{1}\left(X\left(\tau_{k}-\right), X\left(\tau_{k}\right)\right)\right] .
$$

For each $j$, define $\mu_{1, t_{j}}$ by (3.1) and define $G_{n}$ by (5.4). Then

$$
\liminf _{n \rightarrow \infty} \liminf _{j \rightarrow \infty} \int_{\overline{\mathcal{R}}}\left(B G_{n}(y, z)+c_{1}(y, z)\right) \mu_{1, t_{j}}(\mathrm{~d} y \times \mathrm{d} z) \geq 0 .
$$

Proof. Let $(\tau, Y), X$, and $\left\{t_{j}\right\}$ be as in the statement of the proposition. Observe that, for $(y, z) \in \mathcal{R}$,

$$
\begin{aligned}
c_{1}(y, z) & +B G_{n}(y, z) \\
= & c_{1}(y, z)+\frac{G_{0}(z)}{1+h\left(G_{0}(z)\right) / n}-\frac{G_{0}(y)}{1+h\left(G_{0}(y)\right) / n} \\
= & \frac{B G_{0}(y, z)+c_{1}(y, z)}{\left[1+h\left(G_{0}(z)\right) / n\right]\left[1+h\left(G_{0}(y)\right) / n\right]}+\frac{G_{0}(z) h\left(G_{0}(y)\right)-G_{0}(y) h\left(G_{0}(z)\right)}{n\left[1+h\left(G_{0}(z)\right) / n\right]\left[1+h\left(G_{0}(y)\right) / n\right]} \\
& +c_{1}(y, z)\left(1-\frac{1}{\left[1+h\left(G_{0}(z)\right) / n\right]\left[1+h\left(G_{0}(y)\right) / n\right]}\right) \\
\geq & \frac{G_{0}(z) h\left(G_{0}(y)\right)-G_{0}(y) h\left(G_{0}(z)\right)}{n\left[1+h\left(G_{0}(z)\right) / n\right]\left[1+h\left(G_{0}(y)\right) / n\right]} \\
= & : R_{n}(y, z) .
\end{aligned}
$$

The first summand in the middle relation is positive due to Proposition 4.1 and the third summand is easily seen to be positive. This relation also holds on the boundary $y=z$ and $R_{n}(y, y)=0$ for all $y \in \mathcal{E}$. Therefore,

$\liminf _{n \rightarrow \infty} \liminf _{j \rightarrow \infty} \int_{\overline{\mathcal{R}}}\left(B G_{n}+c_{1}\right)(y, z) \mu_{1, t_{j}}(\mathrm{~d} y \times \mathrm{d} z) \geq \liminf _{n \rightarrow \infty} \liminf _{j \rightarrow \infty} \int_{\overline{\mathcal{R}}} R_{n}(y, z) \mu_{1, t_{j}}(\mathrm{~d} y \times \mathrm{d} z)$.

Note that on $\overline{\mathcal{R}}, R_{n}$ takes both positive and negative values.

We examine the double limit of the remainder term $R_{n}$ in several cases. Recall that Condition 2.3 implies the existence of $G_{0}(a)=\lim _{x \rightarrow a} G_{0}(x)$ in $\mathbb{R} \cup\{-\infty\}$ and, similarly, the existence of $G_{0}(b) \in \mathbb{R} \cup\{\infty\}$.

Case $(i)$. $G_{0}(a)>-\infty$ and $G_{0}(b)<\infty$. In this case, $\left|G_{0}(z) h\left(G_{0}(y)\right)-G_{0}(y) h\left(G_{0}(z)\right)\right|$ is bounded by some $K<\infty$ and, hence, $\left|R_{n}(y, z)\right| \leq K / n$. Recalling Remark 3.1, $\left\{\mu_{1, t_{j}}(\overline{\mathcal{R}})\right\}$ is uniformly bounded and, thus,

$$
\liminf _{n \rightarrow \infty} \liminf _{j \rightarrow \infty} \int_{\overline{\mathcal{R}}} R_{n}(y, z) \mu_{1, t_{j}}(\mathrm{~d} y \times \mathrm{d} z) \geq \liminf _{n \rightarrow \infty} \liminf _{j \rightarrow \infty} \int_{\overline{\mathcal{R}}}-\frac{K}{n} \mu_{1, t_{j}}(\mathrm{~d} y \times \mathrm{d} z)=0 .
$$

Case (ii). $G_{0}(a)=-\infty$ and $G_{0}(b)=\infty$. Since $G_{0}(a)=-\infty$, there exists some $y_{1}$, with $y_{1}>a$ such that $G_{0}(x)<-1$ for all $x<y_{1}$. Recall that $h(x)=|x|$ on $(-\infty,-1)$ and $h(x) \geq|x|$ for all $x$. Thus, it follows that, for all $(y, z) \in \mathcal{R}$ with $y \leq y_{1}$,

$$
R_{n}(y, z)=\frac{\left|G_{0}(y)\right|\left(G_{0}(z)+h\left(G_{0}(z)\right)\right)}{n\left[1+h\left(G_{0}(z)\right) / n\right]\left[1+\left|G_{0}(y)\right| / n\right]} \geq 0 .
$$


Similarly, there exists some $z_{1}$ with $z_{1}<b$ such that $G_{0}(x) \geq 1$ for $z_{1}<x<b$ and it follows that, for $(y, z) \in \overline{\mathcal{R}}$ with $z>z_{1}$,

$$
R_{n}(y, z)=\frac{G_{0}(z)\left(h\left(G_{0}(y)\right)-G_{0}(y)\right)}{n\left[1+h\left(G_{0}(z)\right) / n\right]\left[1+h\left(G_{0}(y)\right) / n\right]} \geq 0 .
$$

Define $E_{1}=\left\{(y, z) \in \bar{R}: a<y<y_{1}\right\}$ and $E_{2}=\left\{(y, z) \in \overline{\mathcal{R}}: z>z_{1}\right\}$, so that $R_{n} \geq 0$ on $E_{1} \cup E_{2}$. Observe that $E_{3}:=\overline{\mathcal{R}} \backslash\left(E_{1} \cup E_{2}\right)=\left\{(y, z) \in \overline{\mathcal{R}}: y_{1} \leq y \leq z \leq z_{1}\right\} ; R_{n}$ defined in (5.11) is continuous on this compact set and, therefore, bounded by some $K / n$ as in case (i). It then follows that

$$
\liminf _{n \rightarrow \infty} \liminf _{j \rightarrow \infty} \int_{\overline{\mathcal{R}}} R_{n}(y, z) \mu_{1, t_{j}}(\mathrm{~d} y \times \mathrm{d} z) \geq \liminf _{n \rightarrow \infty} \liminf _{j \rightarrow \infty} \int_{E_{3}} R_{n}(y, z) \mu_{1, t_{j}}(\mathrm{~d} y \times \mathrm{d} z)=0 .
$$

The two remaining cases are handled similarly.

Pulling these results together, we obtain our main theorem.

Theorem 5.1. Assume that Conditions 2.1, 2.2, 2.3, and 5.1 hold. Let $(\tau, Y) \in \mathcal{A}_{0}$ with $J_{0}(\tau, Y)<\infty$. Then

$$
J_{0}(\tau, Y) \geq F_{0}^{*}=F_{0}\left(y_{0}^{*}, z_{0}^{*}\right)=J_{0}\left(\tau^{*}, Y^{*}\right),
$$

in which $\left(\tau^{*}, Y^{*}\right)$ is the ordering policy (2.6) using an optimizing pair $\left(y_{0}^{*}, z_{0}^{*}\right) \in \mathcal{R}$.

Proof. Let $(\tau, Y) \in \mathcal{A}_{0}$ satisfy $J_{0}(\tau, Y)<\infty$. Let $X$ satisfy (1.2), and let $\mu_{0, t}$ and $\mu_{1, t}$ be defined by (3.1) for each $t>0$. Let $\left\{t_{j}\right\}$ be a sequence with $t_{j} \rightarrow \infty$ and

$$
\begin{aligned}
J_{0}(\tau, Y) & =\lim _{j \rightarrow \infty} \frac{1}{t_{j}} \mathbb{E}\left[\int_{0}^{t_{j}} c_{0}(X(s)) \mathrm{d} s+\sum_{k=1}^{\infty} \mathbf{1}_{\left\{\tau_{k} \leq t_{j}\right\}} c_{1}\left(X\left(\tau_{k}-\right), X\left(\tau_{k}\right)\right)\right] \\
& =\lim _{j \rightarrow \infty}\left(\int_{\overline{\mathcal{E}}} c_{0}(x) \mu_{0, t_{j}}(\mathrm{~d} x)+\int_{\overline{\mathcal{R}}} c_{1}(y, z) \mu_{1, t_{j}}(\mathrm{~d} y \times \mathrm{d} z)\right) .
\end{aligned}
$$

The tightness of $\left\{\mu_{0, t_{j}}\right\}$ implies the existence of a weak limit $\mu_{0}$; without loss of generality, assume that $\mu_{0, t_{j}} \Rightarrow \mu_{0}$ as $j \rightarrow \infty$. Proposition 3.2 and its proof establish that

$$
\int_{\bar{\varepsilon}} c_{0} \mathrm{~d} \mu_{0} \leq \liminf _{j \rightarrow \infty} \int_{\bar{\varepsilon}} c_{0} \mathrm{~d} \mu_{0, t_{j}} \leq J_{0}(\tau, Y)<\infty .
$$

Since $G_{n} \in \mathscr{D}, \lim _{j \rightarrow \infty} \int_{\bar{\varepsilon}} A G_{n} \mathrm{~d} \mu_{0, t_{j}}=\int_{\bar{\varepsilon}} A G_{n} \mathrm{~d} \mu_{0}$. Proposition 5.1 implies that, for each $n$,

$$
\lim _{j \rightarrow \infty}\left(\int_{\overline{\mathcal{E}}} A G_{n}(x) \mu_{0}(\mathrm{~d} x)+\int_{\overline{\mathcal{R}}} B G_{n}(y, z) \mu_{1, t_{j}}(\mathrm{~d} y \times \mathrm{d} z)\right)=0,
$$

so adding (5.12) and (5.13) and taking the limit inferior as $n \rightarrow \infty$ yields

$$
\begin{aligned}
J_{0}(\tau, Y)= & \liminf _{n \rightarrow \infty} \lim _{j \rightarrow \infty}\left(\int_{\overline{\mathcal{E}}}\left(A G_{n}(x)+c_{0}(x)\right) \mu_{0, t_{j}}(\mathrm{~d} x)\right. \\
& \left.\quad+\int_{\overline{\mathcal{R}}}\left(B G_{n}(y, z)+c_{1}(y, z)\right) \mu_{1, t_{j}}(\mathrm{~d} y \times \mathrm{d} z)\right) \\
\geq & \liminf _{n \rightarrow \infty} \liminf _{j \rightarrow \infty} \int_{\overline{\mathcal{E}}}\left(A G_{n}(x)+c_{0}(x)\right) \mu_{0, t_{j}}(\mathrm{~d} x) \\
& +\liminf _{n \rightarrow \infty} \liminf _{j \rightarrow \infty} \int_{\overline{\mathcal{R}}}\left(B G_{n}(x)+c_{1}(y)\right) \mu_{1, t_{j}}(\mathrm{~d} y \times \mathrm{d} z)
\end{aligned}
$$




$$
\begin{aligned}
& \geq \liminf _{n \rightarrow \infty} \int_{\overline{\mathcal{E}}}\left(A G_{n}(x)+c_{0}(x)\right) \mu_{0}(\mathrm{~d} x) \\
& \quad+\liminf _{n \rightarrow \infty} \liminf _{j \rightarrow \infty} \int_{\overline{\mathcal{R}}}\left(B G_{n}(x)+c_{1}(y)\right) \mu_{1, t_{j}}(\mathrm{~d} y \times \mathrm{d} z) \\
& \geq F_{0}^{*} ;
\end{aligned}
$$

Propositions 5.2 and 5.3 establish the last inequality.

\section{Examples}

For the cost structure, we consider four examples to illustrate some of the different possible ways in which Proposition 2.1 and Theorem 5.1 can be employed. Some of these cost structures include forms that do not satisfy the modularity condition imposed in Helmes et al. (2017), thus illustrating the breadth of application of the current results.

\subsection{Drifted Brownian motion inventory models}

We begin by examining the classical model that has been studied by Bather (1966), Sulem (1986), Dai and Yao (2013), and many others. In particular, we show that Theorem 5.1 extends the result in He et al. (2017) and verifies optimality as a result of our analytical approach. We then examine a drifted Brownian motion process with reflection at $\{0\}$ using a nontraditional cost structure.

6.1.1. Classical model. In the absence of ordering, the inventory level process $X_{0}$ evolves in the state space $\mathbb{R}$ and satisfies

$$
\mathrm{d} X_{0}(t)=-\mu \mathrm{d} t+\sigma \mathrm{d} W(t), \quad X_{0}(0)=x_{0} \in \ell:=(-\infty, \infty),
$$

in which $\mu, \sigma>0$ and $W$ is a standard Brownian motion process. Observe that the model includes both positive and negative inventory levels indicating real inventory and items that have been back-ordered, respectively.

To specify the cost structure, the holding/back-order cost function $c_{0}$ defined on $\mathbb{R}$ and the ordering cost function $c_{1}$ defined on $\overline{\mathcal{R}}=\left\{(y, z) \in \mathbb{R}^{2}: y \leq z\right\}$ are

$$
c_{0}(x)=\left\{\begin{array}{ll}
-c_{b} x, & x<0, \\
c_{h} x, & x \geq 0,
\end{array} \quad \text { and } \quad c_{1}(y, z)=k_{1}+k_{2}(z-y),\right.
$$

with $c_{b}, c_{h}, k_{1}, k_{2}>0$. The coefficient $c_{b}$ denotes the back-order cost rate per unit of inventory per unit of time while $c_{h}$ is the holding cost rate. The ordering cost function is comprised of fixed plus proportional (to the order size) costs.

Section 4.1 of Helmes et al. (2017) verifies that Conditions 2.1 and 2.2 hold, and proves the existence of an optimizing pair $\left(y_{0}^{*}, z_{0}^{*}\right) \in \mathcal{R}$ such that $F_{0}\left(y_{0}^{*}, z_{0}^{*}\right)=F_{0}^{*}$.

Since $a=-\infty$ is a natural boundary, $\mathcal{A}_{0}=\mathcal{A}$. The following theorem establishes the optimality of the $\left(y_{0}^{*}, z_{0}^{*}\right)$ policy in the class $\mathcal{A}$ without using an ad hoc comparison result as in He et al. (2017).

Theorem 6.1. Let $\left(y_{0}^{*}, z_{0}^{*}\right)$ be an optimizing pair of $F_{0}$. Then the $\left(y_{0}^{*}, z_{0}^{*}\right)$ ordering policy defined by (2.6) is optimal in the class $\mathcal{A}$ for the drifted Brownian motion inventory model under the cost structure (6.2).

Proof. We need to verify that Condition 5.1 holds in order to apply Theorem 5.1 to show that the $\left(y_{0}^{*}, z_{0}^{*}\right)$ ordering policy is optimal. 
First, define the functions $\tilde{g}_{0}$ and $\tilde{\zeta}$ on $\mathbb{R}$ by

$$
\begin{array}{rlr}
\tilde{g}_{0}(x) & =\int_{0}^{x} \int_{u}^{\infty} 2 c_{0}(v) \mathrm{d} M(v) \mathrm{d} S(u) \\
& = \begin{cases}-\frac{c_{b}}{2 \mu} x^{2}-\frac{\sigma^{2} c_{b}}{2 \mu^{2}} x+\frac{\sigma^{4}\left(c_{b}+c_{h}\right)}{4 \mu^{3}}\left(\exp \left\{\frac{2 \mu}{\sigma^{2}} x\right\}-1\right), & x<0, \\
\frac{c_{h}}{2 \mu} x^{2}+\frac{\sigma^{2} c_{h}}{2 \mu^{2}} x, & x \geq 0,\end{cases} \\
\tilde{\zeta}(x) & =\int_{0}^{x} \int_{u}^{\infty} 2 \mathrm{~d} M(v) \mathrm{d} S(u)=\frac{1}{\mu} x .
\end{array}
$$

Note that $\tilde{g}_{0}, \tilde{\zeta} \in C^{2}(\ell)$. It therefore follows from (2.2) that

$$
\begin{aligned}
g_{0}(x) & =\tilde{g}_{0}(x)-\tilde{g}_{0}\left(x_{0}\right) \\
& = \begin{cases}-\frac{c_{b}}{2 \mu} x^{2}-\frac{\sigma^{2} c_{b}}{2 \mu^{2}} x+\frac{\sigma^{4}\left(c_{b}+c_{h}\right)}{4 \mu^{3}}\left(\exp \left\{\frac{2 \mu}{\sigma^{2}} x\right\}-1\right)-\tilde{g}_{0}\left(x_{0}\right), & x<0, \\
\frac{c_{h}}{2 \mu} x^{2}+\frac{\sigma^{2} c_{h}}{2 \mu^{2}} x-\tilde{g}_{0}\left(x_{0}\right), & x \geq 0,\end{cases}
\end{aligned}
$$

and $\zeta(x)=\tilde{\zeta}(x)-\tilde{\zeta}\left(x_{0}\right)=\left(x-x_{0}\right) / \mu$. Defining $\tilde{G}_{0}=\tilde{g}_{0}-F_{0}^{*} \tilde{\zeta}$, it follows that

$$
\begin{aligned}
G_{0}(x)= & \tilde{G}_{0}(x)-\tilde{G}_{0}\left(x_{0}\right) \\
& = \begin{cases}-\frac{c_{b}}{2 \mu} x^{2}-\left(\frac{\sigma^{2} c_{b}}{2 \mu^{2}}+\frac{F_{0}^{*}}{\mu}\right) x+\frac{\sigma^{4}\left(c_{b}+c_{h}\right)}{4 \mu^{3}}\left(\exp \left\{\frac{2 \mu}{\sigma^{2}} x\right\}-1\right) & x<0, \\
-\tilde{G}_{0}\left(x_{0}\right), & x \geq 0 . \\
\frac{c_{h}}{2 \mu} x^{2}+\left(\frac{\sigma^{2} c_{h}}{2 \mu^{2}}-\frac{F_{0}^{*}}{\mu}\right) x-\tilde{G}_{0}\left(x_{0}\right), & \text { i }\end{cases}
\end{aligned}
$$

We examine the cases $x<0$ and $x>0$ separately. For $x>0$, we have the two conditions

$$
\begin{aligned}
& \frac{\left(\sigma(x) G_{0}^{\prime}(x)\right)^{2}}{\left(1+\left|G_{0}(x)\right|\right)\left(1+c_{0}(x)\right)} \\
& =\frac{\left(c_{h} \sigma x / \mu+\sigma^{3} c_{h} /\left(2 \mu^{2}\right)-F_{0}^{*} \sigma / \mu\right)^{2}}{\left(1+\left|c_{h} x^{2} /(2 \mu)+\left(\sigma^{2} c_{h} /\left(2 \mu^{2}\right)-F_{0}^{*} / \mu\right) x-\tilde{G}_{0}\left(x_{0}\right)\right|\right)\left(1+c_{h} x\right)}, \\
& \frac{c_{0}(x)}{\left(1+\left|G_{0}(x)\right|\right)^{2}}=\frac{c_{h} x}{\left(1+\left|c_{h} x^{2} /(2 \mu)+\left(\sigma^{2} c_{h} /\left(2 \mu^{2}\right)-F_{0}^{*} / \mu\right) x-\tilde{G}_{0}\left(x_{0}\right)\right|\right)^{2}},
\end{aligned}
$$

and, hence,

$$
\lim _{x \rightarrow \infty} \frac{\left(\sigma(x) G_{0}^{\prime}(x)\right)^{2}}{\left(1+\left|G_{0}(x)\right|\right)\left(1+c_{0}(x)\right)}=0 \quad \text { and } \quad \lim _{x \rightarrow \infty} \frac{c_{0}(x)}{\left(1+\left|G_{0}(x)\right|\right)^{2}}=0 .
$$

For $x<0$, the ratios are

$$
\begin{aligned}
& \frac{\left(\sigma(x) G_{0}^{\prime}(x)\right)^{2}}{\left(1+\left|G_{0}(x)\right|\right)^{3}} \\
& \quad=\frac{\left(-c_{b} \sigma x / \mu-\left(\sigma^{3} c_{b} /\left(2 \mu^{2}\right)+F_{0}^{*} \sigma / \mu\right)+\left(\sigma^{2}\left(c_{b}+c_{h}\right) /\left(2 \mu^{2}\right)\right) \exp \left\{2 \mu x / \sigma^{2}\right\}\right)^{2}}{\left(1+\left|-c_{b} x^{2} /(2 \mu)-\left(\sigma^{2} c_{b} /\left(2 \mu^{2}\right)+F_{0}^{*} \sigma / \mu\right) x+\left(\sigma^{4}\left(c_{b}+c_{h}\right) /\left(4 \mu^{3}\right)\right)\left(\exp \left\{2 \mu x / \sigma^{2}\right\}-1\right)-\tilde{G}_{0}\left(x_{0}\right)\right|\right)^{3}},
\end{aligned}
$$




$$
\begin{aligned}
& \frac{c_{0}(x)}{\left(1+\left|G_{0}(x)\right|\right)^{2}} \\
& \quad=\frac{-c_{b} x}{\left(1+\left|-c_{b} x^{2} /(2 \mu)-\left(\sigma^{2} c_{b} /\left(2 \mu^{2}\right)+F_{0}^{*} \sigma / \mu\right) x+\left(\sigma^{4}\left(c_{b}+c_{h}\right) /\left(4 \mu^{3}\right)\right)\left(\exp \left\{2 \mu x / \sigma^{2}\right\}-1\right)-\tilde{G}_{0}\left(x_{0}\right)\right|\right)^{2}},
\end{aligned}
$$

yielding

$$
\lim _{x \rightarrow-\infty} \frac{\left(\sigma(x) G_{0}^{\prime}(x)\right)^{2}}{\left(1+\left|G_{0}(x)\right|\right)^{3}}=0 \quad \text { and } \quad \lim _{x \rightarrow-\infty} \frac{c_{0}(x)}{\left(1+\left|G_{0}(x)\right|\right)^{2}}=0 .
$$

As a result, both ratios are uniformly bounded and the result holds.

6.1.2. Drifted Brownian motion with reflection at $\{0\}$. For this model, $X$ is a drifted Brownian motion process that reflects at $\{0\}$. Thus, when $X(t)>0, X$ follows the dynamics in (6.1) and Condition 2.1 is established in Section 4.1 of Helmes et al. (2017).

The cost structure is given by the functions $c_{0}$ on $[0, \infty)$ and $c_{1}$ on $\{(y, z): 0 \leq y \leq z\}$ by

$$
c_{0}(x)=k_{3} x+k_{4} \mathrm{e}^{-x} \quad \text { and } \quad c_{1}(y, z)=k_{1}+k_{2} \sqrt{z-y},
$$

with $k_{1}, k_{2}, k_{3}, k_{4}>0$. Note that the holding costs for small inventory levels are penalized by the term $k_{4} \mathrm{e}^{-x}$, which remains bounded at 0 . Also, the ordering cost function is a concave function of the order size, so incorporates savings due to economies of scale. This $c_{1}$ function does not satisfy the critical modularity condition required in Helmes et al. (2017). We begin by establishing the existence of a pair of minimizers for $F_{0}$.

The analysis below verifies the existence of an optimal $(s, S)$ ordering policy in the class $\mathcal{A}_{0}$ which does not allow any positive amount of long-term average expected local time of the inventory process at $\{0\}$.

Proposition 6.1. There exists an optimizing pair $\left(y_{0}^{*}, z_{0}^{*}\right) \in \mathcal{R}$ of $F_{0}$ for the drifted Brownian motion model with reflection having cost structure (6.4).

Proof. Verification of Condition 2.2 follows by straightforward computations, so is left to the reader. By the assumption on the model, the boundary 0 is regular (reflective), while the boundary $\infty$ is natural with $c_{0}(\infty)=\infty$. Thus, Condition 2.3 holds and an application of Theorem 2.1 establishes the result.

Theorem 6.2. Let $\left(y_{0}^{*}, z_{0}^{*}\right)$ be as in Proposition 6.1. Then the $\left(y_{0}^{*}, z_{0}^{*}\right)$ ordering policy is optimal in the class $\mathcal{A}_{0}$ for the reflected drifted Brownian motion inventory model under the cost structure (6.4).

Proof. It suffices to show that Condition 5.1 holds. The proof of Theorem 6.1 shows that $\zeta(x)=\tilde{\zeta}(x)-\tilde{\zeta}\left(x_{0}\right)$ with $\tilde{\zeta}(x)=x / \mu$ and, as in the same proof, straightforward computation establishes that

$$
\tilde{g}_{0}(x)=\frac{k_{3}}{2 \mu} x^{2}+\frac{k_{3} \sigma^{2}}{2 \mu^{2}} x+\frac{2 k_{4} \sigma^{2}}{\left(2 \mu+\sigma^{2}\right)^{2}}\left(1-\mathrm{e}^{-x}\right),
$$

so $g_{0}(x)=\tilde{g}_{0}(x)-\tilde{g}_{0}\left(x_{0}\right)$. Setting $\tilde{G}_{0}=\tilde{g}_{0}-F_{0}^{*} \tilde{\zeta}$, the function $G_{0}$ is

$$
G_{0}(x)=\frac{k_{3}}{2 \mu} x^{2}+\left(\frac{k_{3} \sigma^{2}}{2 \mu^{2}}-\frac{F_{0}^{*}}{\mu}\right) x+\frac{2 k_{4} \sigma^{2}}{\left(2 \mu+\sigma^{2}\right)^{2}}\left(1-\mathrm{e}^{-x}\right)-\tilde{G}_{0}\left(x_{0}\right) .
$$

At the left boundary $0, c_{0}(0)=k_{4}$ and $G_{0}(0)=2 k_{4} \sigma^{2} /\left(2 \mu+\sigma^{2}\right)^{2}-\tilde{G}_{0}\left(x_{0}\right)$. It immediately follows from continuity that Condition 5.1(a)(ii) holds and direct computation verifies that Condition 5.1(c)(ii) is satisfied. We thus need to examine the ratios of Condition 5.1 when $x$ is large. These ratios differ only slightly from (6.3) (equating $c_{h}$ with $k_{3}$ ) and the exponential term does not affect the limits. Thus, Condition 5.1 holds and Theorem 5.1 then yields the results. 
Remark 6.1. Consider models in which $\left(k_{1} \vee\left(k_{2} / 2\right)\right) \wedge k_{3} /(2 \mu) \geq 2 k_{4} \sigma^{2} /\left(2 \mu+\sigma^{2}\right)^{2}$, so that either the fixed or unit ordering cost is expensive relative to the holding cost rate near 0 and the linear holding cost rate is expensive relative to this same holding cost rate near 0 . Taking the partial derivative with respect to $y$ yields

$$
\begin{gathered}
\frac{\partial F_{0}}{\partial y}(y, z)=\frac{\mu}{z-y}\left[\frac{k_{1}}{z-y}+\frac{k_{2}}{2 \sqrt{z-y}}+\frac{k_{3}}{2 \mu}(z-y)-\frac{2 k_{4} \sigma^{2}}{\left(2 \mu+\sigma^{2}\right)^{2}} \mathrm{e}^{-y}\right. \\
\left.+\frac{2 k_{4} \sigma^{2}}{\left(2 \mu+\sigma^{2}\right)^{2}} \frac{\mathrm{e}^{-y}-\mathrm{e}^{-z}}{z-y}\right] .
\end{gathered}
$$

Since $y \geq 0$, observe that

- for all $(y, z) \in \mathcal{R}$ with $z-y \leq 1, k_{1} /(z-y) \geq 2 k_{4} \sigma^{2} \mathrm{e}^{-y} /\left(2 \mu+\sigma^{2}\right)^{2}$ or $k_{2} /(2 \sqrt{z-y}) \geq$ $2 k_{4} \sigma^{2} \mathrm{e}^{-y} /\left(2 \mu+\sigma^{2}\right)^{2} ;$ and

- $k_{3}(z-y) / 2 \mu \geq 2 k_{4} \sigma^{2} \mathrm{e}^{-y} /\left(2 \mu+\sigma^{2}\right)^{2}$ for all $(y, z) \in \mathcal{R}$ with $z-y>1$.

The positivity of the last term in the brackets of $\partial F_{0} / \partial y$ therefore implies that $\partial F_{0}(y, z) / \partial y>0$ for all $(y, z) \in \mathcal{R}$ and, hence, $y_{0}^{*}=0$. Thus, the optimal ordering policy waits until the inventory hits 0 before ordering. (The optimal level $z_{0}^{*}$ is determined from the transcendental equation obtained by setting $\partial F_{0}(0, z) / \partial z=0$.)

\subsection{Geometric Brownian motion storage model}

Without any ordering, the inventory process is a geometric Brownian motion evolving in the state space $(0, \infty)$ and satisfying the stochastic differential equation

$$
\mathrm{d} X_{0}(t)=-\mu X_{0}(t) \mathrm{d} t+\sigma X_{0}(t) \mathrm{d} W(t), \quad X(0)=x_{0} \in \ell=(0, \infty),
$$

in which $\mu, \sigma>0$ and $W$ is a standard Brownian motion process; the drift rate is negative. It is well known that both boundaries are natural. Section 4.2 of Helmes et al. (2017) shows that this model satisfies Condition 2.1.

6.2.1. Nonlinear holding costs with ordering costs that are a concave function of the order size. The cost functions $c_{0}$ on $(0, \infty)$ and $c_{1}$ on $\{(y, z): 0<y \leq z\}$ are given by

$$
c_{0}(x)=k_{3} x+k_{4} x^{\beta} \quad \text { and } \quad c_{1}(y, z)=k_{1}+k_{2} \sqrt{z-y},
$$

with $k_{1}, k_{2}, k_{3}, k_{4}>0$ and $\beta<0$. For this $c_{0}$ function, $k_{4} x^{\beta}$ is extremely costly as the level approaches 0 . The proof of the next lemma is straightforward, so is left to the reader.

Lemma 6.1. Condition 2.2 holds for this geometric Brownian motion storage model having cost structure (6.5).

We now turn to the existence of an optimal $(s, S)$ ordering policy.

Theorem 6.3. There exists an optimal $\left(y_{0}^{*}, z_{0}^{*}\right)$ ordering policy in the class $\mathcal{A}$ for the geometric Brownian motion storage model having nonlinear cost structure given by (6.5).

Proof. By Theorem 5.1, it suffices to show that Conditions 2.3 and 5.1 hold. As previously noted, 0 and $\infty$ are natural boundaries, and we see that $c_{0}(0)=\infty$ and $c_{0}(\infty)=\infty$, so Condition 2.3(a)(i) and (b)(i) hold.

Let $\rho=\sigma^{2} \beta^{2} / 2-\mu \beta$, and note that $\rho>0$. Straightforward computations using (2.2) determine

$$
g_{0}(x)=\frac{k_{3}}{\mu}\left(x-x_{0}\right)-\frac{k_{4}}{\rho}\left(x^{\beta}-x_{0}^{\beta}\right) \quad \text { and } \quad \zeta(x)=\frac{2}{2 \mu+\sigma^{2}}\left(\ln (x)-\ln \left(x_{0}\right)\right), \quad x \in \ell,
$$


and, hence,

$$
G_{0}(x)=\frac{k_{3}}{\mu}\left(x-x_{0}\right)-\frac{k_{4}}{\rho}\left(x^{\beta}-x_{0}^{\beta}\right)-\frac{2 F_{0}^{*}}{2 \mu+\sigma^{2}}\left(\ln (x)-\ln \left(x_{0}\right)\right) .
$$

As a result, we have

$$
\begin{aligned}
& \frac{\left(\sigma(x) G_{0}^{\prime}(x)\right)^{2}}{\left(1+\left|G_{0}(x)\right|\right)^{3}} \\
& \quad=\frac{\left(k_{3} \sigma x / \mu+k_{4} \sigma(-\beta) x^{\beta} / \rho-2 F_{0}^{*} /\left(2 \mu+\sigma^{2}\right)\right)^{2}}{\left(1+\left|k_{3}\left(x-x_{0}\right) / \mu-k_{4}\left(x^{\beta}-x_{0}^{\beta}\right) / \rho-\left(2 F_{0}^{*} /\left(2 \mu+\sigma^{2}\right)\right)\left(\ln (x)-\ln \left(x_{0}\right)\right)\right|\right)^{3}},
\end{aligned}
$$

so at the left boundary $a=0$,

$$
\lim _{x \rightarrow 0} \frac{\left(\sigma(x) G_{0}^{\prime}(x)\right)^{2}}{\left(1+\left|G_{0}(x)\right|\right)^{3}}=0 .
$$

Turning to the boundary $b=\infty$,

$$
\begin{aligned}
& \frac{\left(\sigma(x) G_{0}^{\prime}(x)\right)^{2}}{\left(1+\left|G_{0}(x)\right|\right)\left(1+c_{0}(x)\right)} \\
& \quad=\frac{\left(k_{3} \sigma x / \mu+k_{4} \sigma(-\beta) x^{\beta} / \rho-2 F_{0}^{*} /\left(2 \mu+\sigma^{2}\right)\right)^{2}}{\left(1+\left|k_{3}\left(x-x_{0}\right) / \mu-k_{4}\left(x^{\beta}-x_{0}^{\beta}\right) / \rho-\left(2 F_{0}^{*} /\left(2 \mu+\sigma^{2}\right)\right)\left(\ln (x)-\ln \left(x_{0}\right)\right)\right|\right)\left(1+k_{3} x+k_{4} x^{\beta}\right)},
\end{aligned}
$$

so

$$
\lim _{x \rightarrow \infty} \frac{\left(\sigma(x) G_{0}^{\prime}(x)\right)^{2}}{\left(1+\left|G_{0}(x)\right|\right)\left(1+c_{0}(x)\right)}=\frac{\sigma^{2}}{\mu} .
$$

Finally, we have

$$
\begin{aligned}
& \frac{c_{0}(x)}{\left(1+\left|G_{0}(x)\right|\right)^{2}} \\
& \quad=\frac{k_{3} x+k_{4} x^{\beta}}{\left(1+\left|k_{3}\left(x-x_{0}\right) / \mu-k_{4}\left(x^{\beta}-x_{0}^{\beta}\right) / \rho-\left(2 F_{0}^{*} /\left(2 \mu+\sigma^{2}\right)\right)\left(\ln (x)-\ln \left(x_{0}\right)\right)\right|\right)^{2}},
\end{aligned}
$$

which gives

$$
\lim _{x \rightarrow 0} \frac{c_{0}(x)}{\left(1+\left|G_{0}(x)\right|\right)^{2}}=0 \text { and } \lim _{x \rightarrow \infty} \frac{c_{0}(x)}{\left(1+\left|G_{0}(x)\right|\right)^{2}}=0 .
$$

As a result, (6.6), (6.7), and (6.8) imply that Condition 5.1(a)(i) and (b)(i) hold.

Remark 6.2. When $k_{4}=0$ in (6.5), the conditions of Proposition 2.1(a) hold, so there does not exist any optimal $(s, S)$ ordering policy and the 'no order' policy is optimal. See also Section 4.2.2 of Helmes et al. (2017) for an alternate analysis.

6.2.2. Piecewise linear holding costs with modular ordering costs. The cost functions $c_{0}$ on $[0, \infty)$ and $c_{1}$ on $\left\{(y, z) \in \mathbb{R}_{+}^{2}: y \leq z\right\}$ are given by

$$
\begin{gathered}
c_{0}(x)= \begin{cases}k_{4}(1-x), & 0 \leq x \leq 1, \\
k_{3}(x-1), & x \geq 1,\end{cases} \\
c_{1}(y, z)=k_{1}+\frac{1}{2} k_{2}\left(y^{-1 / 2}-z^{-1 / 2}\right)+\frac{1}{2} k_{2}(z-y),
\end{gathered}
$$

with $k_{1}, k_{2}, k_{3}>0$ and $k_{4}>\left(\sigma^{2}+2 \mu\right)\left[k_{1}+k_{2}\left(1-\mathrm{e}^{-1 / 2}\right) / 2+\left(k_{2} \mu+2 k_{3}\right)(\mathrm{e}-1) /(2 \mu)\right]-2 k_{3}$. Note that $\lim _{y \rightarrow 0} c_{1}(y, z)=\infty$, placing a very strong penalty on waiting until the inventory 
level is nearly 0 . In this example, the holding cost rate decreases at rate $k_{4}$ on $[0,1]$ and increases at rate $k_{3}$ thereafter. The function $c_{1}$ is modular in the sense that, for any $0<w \leq x \leq y \leq z$, $c_{1}(x, z)-c_{1}(x, y)-c_{1}(w, z)+c_{1}(w, y)=0$. By writing $c_{1}$ in the form

$$
c_{1}(y, z)=k_{1}+k_{2}\left(\frac{\left(y^{-1 / 2}-z^{-1 / 2}\right) /(z-y)+1}{2}\right)(z-y),
$$

the cost per unit portion of the charge is smaller with large values of $y$ and $z$, so encourages the decision maker to order before the inventory falls very low and prefers large orders.

We note that the modularity condition on $c_{1}$ is a critical condition in Helmes et al. (2017). That paper also requires $c_{0}(x) \rightarrow \infty$ as $x \rightarrow 0$, which is violated in (6.9).

The proof of the next result is straightforward and left to the reader.

Lemma 6.2. The geometric Brownian motion model with cost structure given in (6.9) satisfies Condition 2.2.

Proposition 6.2. The geometric Brownian motion model with cost functions given by (6.9) satisfies Condition 2.3 and, hence, there exists an optimizing pair $\left(y_{0}^{*}, z_{0}^{*}\right) \in \mathcal{R}$ of the function $F_{0}$.

Proof. We begin by defining the functions $\tilde{\zeta}(x)=2 \ln (x) /\left(2 \mu+\sigma^{2}\right), x \in(0, \infty)$, and $\tilde{g}_{0}$ on $(0, \infty)$ by

$$
\begin{aligned}
\tilde{g}_{0}(x) & =\int_{1}^{x} \int_{u}^{\infty} 2 c_{0}(v) \mathrm{d} M(v) \mathrm{d} S(u) \\
& = \begin{cases}\frac{2 k_{4}}{2 \mu+\sigma^{2}} \ln (x)-\frac{k_{4}}{\mu}(x-1)+\frac{\sigma^{4}\left(k_{3}+k_{4}\right)}{\mu\left(2 \mu+\sigma^{2}\right)^{2}}\left(x^{\left(2 \mu+\sigma^{2}\right) / \sigma^{2}}-1\right), & x<1, \\
\frac{k_{3}}{\mu}(x-1)-\frac{2 k_{3}}{2 \mu+\sigma^{2}} \ln (x), & x \geq 1 .\end{cases}
\end{aligned}
$$

It then follows from $(2.2)$ that $g_{0}(x)=\tilde{g}_{0}(x)-\tilde{g}_{0}\left(x_{0}\right)$ and $\zeta(x)=\tilde{\zeta}(x)-\tilde{\zeta}\left(x_{0}\right)$. Note that $g_{0}^{\prime}=\tilde{g}_{0}^{\prime}$ and $\zeta^{\prime}=\tilde{\zeta}^{\prime}$.

Since 0 is a natural boundary and $c_{0}(0)=k_{4}<\infty$, it suffices to show that, for each $z \in(0, \infty)$, there exists $0<y_{z}<z$ such that, for all $0<y<y_{z}$,

$$
\frac{-\partial c_{1}(y, z) / \partial y+g_{0}^{\prime}(y)}{\zeta^{\prime}(y)} \geq F_{0}(y, z)
$$

and to show the existence of $(\bar{y}, \bar{z})$ for which $F_{0}(\bar{y}, \bar{z})<k_{4}$. Arbitrarily fix $z \in(0, \infty)$. Since we need $y$ sufficiently close to 0 , using $y<1$ gives

$$
\begin{aligned}
& \frac{-\partial c_{1}(y, z) / \partial y+g_{0}^{\prime}(y)}{\zeta^{\prime}(y)} \\
& =\frac{k_{2}\left(y^{-3 / 2}-1\right) / 2+2 k_{4} y^{-1} /\left(2 \mu+\sigma^{2}\right)-k_{4} / \mu+\sigma^{2}\left(k_{3}+k_{4}\right) y^{2 \mu / \sigma^{2}} /\left(\mu\left(2 \mu+\sigma^{2}\right)\right)}{2 y^{-1} /\left(2 \mu+\sigma^{2}\right)} \\
& \quad=\frac{k_{2}\left(2 \mu+\sigma^{2}\right)}{4}\left(y^{-1 / 2}-y\right)+k_{4}+\frac{k_{4}\left(2 \mu+\sigma^{2}\right)}{2 \mu} y+\frac{\sigma^{2}\left(k_{3}+k_{4}\right)}{2 \mu} y^{\left(2 \mu+\sigma^{2}\right) / \sigma^{2}} .
\end{aligned}
$$


Similarly,

$$
\begin{aligned}
F_{0}(y, z)= & \frac{k_{1}+k_{2}\left(y^{-1 / 2}-z^{-1 / 2}\right) / 2+k_{2}(z-y) / 2}{2(\ln (z)-\ln (y)) /\left(2 \mu+\sigma^{2}\right)} \\
& +\frac{g_{0}(z)-2 k_{4} \ln (y) /\left(2 \mu+\sigma^{2}\right)+k_{4}(y-1) / \mu-\sigma^{4}\left(k_{3}+k_{4}\right)\left(y^{\left(2 \mu+\sigma^{2}\right) / \sigma^{2}}-1\right) /\left(\mu\left(2 \mu+\sigma^{2}\right)^{2}\right)}{2(\ln (z)-\ln (y)) /\left(2 \mu+\sigma^{2}\right)} \\
= & k_{4}+\frac{k_{1}+k_{2}\left(y^{-1 / 2}-z^{-1 / 2}\right) / 2+k_{2}(z-y) / 2}{2(\ln (z)-\ln (y)) /\left(2 \mu+\sigma^{2}\right)} \\
& +\frac{g_{0}(z)-2 k_{4} \ln (z) /\left(2 \mu+\sigma^{2}\right)+k_{4}(y-1) / \mu-\sigma^{4}\left(k_{3}+k_{4}\right)\left(y^{\left(2 \mu+\sigma^{2}\right) / \sigma^{2}}-1\right) /\left(\mu\left(2 \mu+\sigma^{2}\right)^{2}\right)}{2(\ln (z)-\ln (y)) /\left(2 \mu+\sigma^{2}\right)} .
\end{aligned}
$$

Since the leading order terms as $y \rightarrow 0$ are $y^{-1 / 2}$ and $y^{-1 / 2} / \ln (y)$, respectively, it follows that

$$
\lim _{y \rightarrow 0} \frac{F_{0}(y, z)}{\left(-\partial c_{1}(y, z) / \partial y+g_{0}^{\prime}(y)\right) / \zeta^{\prime}(y)}=0
$$

and, hence, there exists some $y_{z}>a$ so that (2.11) holds for all $a<y<y_{z}$.

Finally, observe that the choice $(\bar{y}, \bar{z})=(1, \mathrm{e})$ has

$$
F_{0}(1, \mathrm{e})=\left(\sigma^{2}+2 \mu\right)\left[k_{1}+\frac{k_{2}}{2}\left(1-\mathrm{e}^{-1 / 2}\right)+\frac{k_{2} \mu+2 k_{3}}{2 \mu}(\mathrm{e}-1)\right]-2 k_{3}<k_{4}
$$

by the model restriction on $k_{4}$.

Theorem 6.4. There exists an optimal $\left(y_{0}^{*}, z_{0}^{*}\right)$ ordering policy in the class $\mathcal{A}$ for the geometric Brownian motion model having nonlinear cost structure given by (6.9).

Proof. The existence of $\left(y_{0}^{*}, z_{0}^{*}\right) \in \mathcal{R}$ with $F_{0}\left(y_{0}^{*}, z_{0}^{*}\right)=F_{0}^{*}$ follows from Proposition 6.2. To apply Theorem 5.1, we need to show that Condition 5.1 holds.

Defining $\tilde{G}_{0}=\tilde{g}_{0}-F_{0}^{*} \tilde{\zeta}$, it follows that $G_{0}(x)=\tilde{G}_{0}(x)-\tilde{G}_{0}\left(x_{0}\right)$ and so $G_{0}^{\prime}(x)=$ $\tilde{g}_{0}^{\prime}(x)-F_{0}^{*} \tilde{\zeta}^{\prime}(x)$. As a result, for sufficiently large $x$, we have

$$
\begin{gathered}
\left(\sigma(x) G_{0}^{\prime}(x)\right)^{2}=\left(\frac{\sigma k_{3}}{\mu} x-\frac{2\left(\sigma k_{3}+F_{0}^{*}\right)}{2 \mu+\sigma^{2}}\right)^{2} \\
1+\left|G_{0}(x)\right|=1+\frac{k_{3}}{\mu}(x-1)-\frac{2\left(k_{3}+F_{0}^{*}\right)}{2 \mu+\sigma^{2}} \ln (x)-\tilde{g}_{0}\left(x_{0}\right), \\
c_{0}(x)=k_{3}(x-1) .
\end{gathered}
$$

Thus,

$$
\lim _{x \rightarrow \infty} \frac{\left(\sigma(x) G_{0}^{\prime}(x)\right)^{2}}{\left(1+\left|G_{0}(x)\right|\right)\left(1+c_{0}(x)\right)}=\frac{\sigma^{2}}{\mu} \quad \text { and } \quad \lim _{x \rightarrow \infty} \frac{\left(\sigma(x) G_{0}^{\prime}(x)\right)^{2}}{\left(1+\left|G_{0}(x)\right|\right)^{2+\varepsilon}}=0 .
$$

For $x$ in a neighborhood of the left boundary 0 ,

$$
\begin{gathered}
G_{0}(x)=\frac{2\left(k_{4}-F_{0}^{*}\right)}{2 \mu+\sigma^{2}} \ln (x)-\frac{k_{4}}{\mu}(x-1)+\frac{\sigma^{4}\left(k_{3}+k_{4}\right)}{\mu\left(2 \mu+\sigma^{2}\right)^{2}}\left(x^{\left(2 \mu+\sigma^{2}\right) / \sigma^{2}}-1\right)-\tilde{g}_{0}\left(x_{0}\right), \\
\left(\sigma(x) G_{0}^{\prime}(x)\right)^{2}=\left(-\frac{\sigma^{2}\left(\sigma^{2} k_{4}-2 \mu F_{0}^{*}\right)}{\mu\left(2 \mu+\sigma^{2}\right)} x+\frac{\sigma^{4}\left(k_{3}+k_{4}\right)}{\mu\left(2 \mu+\sigma^{2}\right)} x^{\left(2 \mu+\sigma^{2}\right) / \sigma^{2}}\right)^{2} .
\end{gathered}
$$

It follows that $\lim _{x \rightarrow 0} G_{0}(x)=-\infty$ and $\lim _{x \rightarrow 0}\left(\sigma^{2}(x) G_{0}^{\prime}(x)\right)^{2}=0$, which then implies that Condition 5.1(a) is satisfied. 


\section{Appendix A. Proofs for Section 2}

Proof of Lemma 2.1. The arguments related to the right boundary $b$ are very similar to and a bit more straightforward than those related to the left boundary $a$. We therefore leave the verification of the limits at $b$ to the reader.

Similarly, the argument when $c_{0}(a)=\infty$ is essentially the same (using the definition of a limit being $\infty$ ) as when $c_{0}(a)<\infty$, so we give the details in the latter instance. Choose $\varepsilon>0$ arbitrarily, and let $y_{\varepsilon} \in \ell$ be such that, for all $a<x<y_{\varepsilon},\left|c_{0}(x)-c_{0}(a)\right|<\varepsilon$. For $a<y<y_{\varepsilon}$, define $\tau_{y}$ to be the first hitting time of $\{y\}$ by the process $X_{0}$ of $(1.1)$ when $X_{0}(0)=y_{\varepsilon}$. Since $a$ is a natural boundary, $\lim _{y \rightarrow a}\left(\zeta\left(y_{\varepsilon}\right)-\zeta(y)\right)=\lim _{y \rightarrow a} \mathbb{E}_{y_{\varepsilon}}\left[\tau_{y}\right]=\infty$. Using the definition of $\zeta$ in (2.2), observe that

$$
\zeta\left(y_{\varepsilon}\right)-\zeta(y)=\int_{y}^{y_{\varepsilon}} \int_{u}^{b} 2 \mathrm{~d} M(v) \mathrm{d} S(u)=\int_{y}^{y_{\varepsilon}} \int_{u}^{y_{\varepsilon}} 2 \mathrm{~d} M(v) \mathrm{d} S(u)+2 M\left[y_{\varepsilon}, b\right) S\left[y, y_{\varepsilon}\right] .
$$

By Condition 2.1(b), $M\left[y_{\varepsilon}, b\right)<\infty$ and, since $a$ is attracting, $\lim _{y \rightarrow a} S\left[y, y_{\varepsilon}\right]=S\left(a, y_{\varepsilon}\right]<\infty$. Thus, the limit of the double integral as $y \rightarrow a$ is infinite.

Similarly to (A.1), using the definition of $g_{0}$ in (2.2), we have

$$
g_{0}\left(y_{\varepsilon}\right)-g_{0}(y)=\int_{y}^{y_{\varepsilon}} \int_{u}^{y_{\varepsilon}} 2 c_{0}(v) \mathrm{d} M(v) \mathrm{d} S(u)+2\left(\int_{y_{\varepsilon}}^{b} c_{0}(v) \mathrm{d} M(v)\right) S\left[y, y_{\varepsilon}\right] .
$$

Due to the integrability condition (2.1) on $c_{0}$ relative to the speed measure $M$, the second term on the right-hand side remains bounded as $y \rightarrow a$. Therefore, writing

$$
\frac{g_{0}\left(y_{\varepsilon}\right)-g_{0}(y)}{\zeta\left(y_{\varepsilon}\right)-\zeta(y)}=\frac{\int_{y}^{y_{\varepsilon}} \int_{u}^{y_{\varepsilon}} 2 c_{0}(v) \mathrm{d} M(v) \mathrm{d} S(u)}{\zeta\left(y_{\varepsilon}\right)-\zeta(y)}+\frac{2\left(\int_{y_{\varepsilon}}^{b} c_{0}(v) \mathrm{d} M(v)\right) S\left[y, y_{\varepsilon}\right]}{\zeta\left(y_{\varepsilon}\right)-\zeta(y)},
$$

the second summand converges to 0 as $y \rightarrow a$, so the asymptotics are determined by the first summand. Using (A.1), observe that

$$
\begin{aligned}
& \frac{\int_{y}^{y_{\varepsilon}} \int_{u}^{y_{\varepsilon}} 2 c_{0}(v) \mathrm{d} M(v) \mathrm{d} S(u)}{\zeta\left(y_{\varepsilon}\right)-\zeta(y)} \\
& \quad=\left(\frac{\int_{y}^{y_{\varepsilon}} \int_{u}^{y_{\varepsilon}} 2 c_{0}(v) \mathrm{d} M(v) \mathrm{d} S(u)}{\int_{y}^{y_{\varepsilon}} \int_{u}^{y_{\varepsilon}} 2 \mathrm{~d} M(v) \mathrm{d} S(u)}\right)\left(\frac{1}{1+2 M\left[y_{\varepsilon}, b\right) S\left[y, y_{\varepsilon}\right] / \int_{y}^{y_{\varepsilon}} \int_{u}^{y_{\varepsilon}} 2 \mathrm{~d} M(v) \mathrm{d} S(u)}\right) .
\end{aligned}
$$

The second factor converges to 1 as $y \rightarrow a$, so by the choice of $y_{\varepsilon}$ we have

$$
c_{0}(a)-\varepsilon \leq \liminf _{y \rightarrow a} \frac{g_{0}\left(y_{\varepsilon}\right)-g_{0}(y)}{\zeta\left(y_{\varepsilon}\right)-\zeta(y)} \leq \limsup _{y \rightarrow a} \frac{g_{0}\left(y_{\varepsilon}\right)-g_{0}(y)}{\zeta\left(y_{\varepsilon}\right)-\zeta(y)} \leq c_{0}(a)+\varepsilon .
$$

Recall that $\zeta(y) \rightarrow-\infty$ as $y \rightarrow a$. In the case $g_{0}(y)$ remains bounded as $y \rightarrow a$, then $\lim _{y \rightarrow a} g_{0}(y) / \zeta(y)=0$. Moreover, the limit exists in (A.2) and equals 0 , so

$$
c_{0}(a)-\varepsilon \leq \lim _{y \rightarrow a} \frac{g_{0}\left(y_{\varepsilon}\right)-g_{0}(y)}{\zeta\left(y_{\varepsilon}\right)-\zeta(y)}=0 \leq c_{0}(a)+\varepsilon .
$$

Since $\varepsilon$ is arbitrary, it follows that $c_{0}(a)=0$ and, hence, (2.5) holds. 
When $g_{0}(y) \rightarrow-\infty$ as $y \rightarrow a$, we have

$$
c_{0}(a)-\varepsilon \leq \liminf _{y \rightarrow a}\left(\left(\frac{g_{0}(y)}{\zeta(y)}\right)\left(\frac{\left(g_{0}\left(y_{\varepsilon}\right) / g_{0}(y)\right)-1}{\left(\zeta\left(y_{\varepsilon}\right) / \zeta(y)\right)-1}\right)\right)=\liminf _{y \rightarrow a} \frac{g_{0}(y)}{\zeta(y)},
$$

and, similarly,

$$
\limsup _{y \rightarrow a} \frac{g_{0}(y)}{\zeta(y)} \leq c_{0}(a)+\varepsilon
$$

Since $\varepsilon$ is arbitrary, it again follows that $\lim _{y \rightarrow a} g_{0}(y) / \zeta(y)=c_{0}(a)$, establishing (2.5). Picking $z \in \&$ arbitrarily, identity (2.3) now follows.

A similar argument addresses (2.4). Again, using the definitions of $g_{0}$ and $\zeta$, we have

$$
\begin{aligned}
& \frac{g_{0}(z)-g_{0}(y)}{\zeta(z)-\zeta(y)} \\
& \quad=\frac{\int_{y}^{z} \int_{u}^{y_{\varepsilon}} 2 c_{0}(v) \mathrm{d} M(v) \mathrm{d} S(u)+\left(\int_{y_{\varepsilon}}^{b} 2 c_{0}(v) \mathrm{d} M(v)\right) S[y, z]}{\int_{y}^{z} 2 M\left[u, y_{\varepsilon}\right] \mathrm{d} S(u)+2 M\left[y_{\varepsilon}, b\right) S[y, z]} \\
& \quad=\left(\frac{\int_{y}^{z} \int_{u}^{y_{\varepsilon}} 2 c_{0}(v) \mathrm{d} M(v) \mathrm{d} S(u)}{\int_{y}^{z} \int_{u}^{y_{\varepsilon}} 2 \mathrm{~d} M(v) \mathrm{d} S(u)}+\frac{\left(\int_{y_{\varepsilon}}^{b} 2 c_{0}(v) \mathrm{d} M(v)\right) S[y, z]}{\int_{y}^{z} 2 M\left[u, y_{\varepsilon}\right] \mathrm{d} S(u)}\right) /\left(1+\frac{2 M\left[y_{\varepsilon}, b\right) S[y, z]}{\int_{y}^{z} 2 M\left[u, y_{\varepsilon}\right] \mathrm{d} S(u)}\right) .
\end{aligned}
$$

Since $a$ is attracting, $S(a)<\infty$, and since it is a natural boundary, $\zeta(a)=-\infty$. Together, these imply that $\lim _{y \rightarrow a} M[y, z]=\infty$; this holds, in particular, when $z=y_{\varepsilon}$. Examining the second summands in both the numerator and denominator above, set $K$ below to be $K=M\left[y_{\varepsilon}, b\right)$ for the denominator term and $K=\int_{y_{\varepsilon}}^{b} c_{0}(v) \mathrm{d} M(v)$ for the numerator summand. Then

$$
\frac{2 K S[y, z]}{\int_{y}^{z} 2 M\left[u, y_{\varepsilon}\right] \mathrm{d} S(u)} \leq \frac{2 K S[y, z]}{2 M\left[z, y_{\varepsilon}\right] S[y, z]}
$$

and the right-hand side converges to 0 as $z \rightarrow a$. Thus, for all $y<z \leq y_{\varepsilon}$, $c_{0}(a)-\varepsilon \leq \liminf _{(y, z) \rightarrow(a, a)} \frac{g_{0}(z)-g_{0}(y)}{\zeta(z)-\zeta(y)} \leq \limsup _{(y, z) \rightarrow(a, a)} \frac{\int_{y}^{z} \int_{u}^{y_{\varepsilon}} 2 c_{0}(v) \mathrm{d} M(v) \mathrm{d} S(u)}{\int_{y}^{z} \int_{u}^{y_{\varepsilon}} 2 \mathrm{~d} M(v) \mathrm{d} S(u)} \leq c_{0}(a)+\varepsilon$, and the result follows since $\varepsilon$ is arbitrary.

Proof of Theorem 2.1. We examine the behavior of $F_{0}$ at each boundary using the same order of analysis as in the proof of Proposition 3.5 of Helmes et al. (2017).

Case (i): the diagonal $z=y$, excluding natural boundaries. By definition, $F_{0}(y, y)=\infty$, which is not the minimal value of $F_{0}$.

Case (ii): the boundary $z=b$, excluding $(a, b)$ when $a$ is natural. Suppose that $b$ is an entrance boundary, so $\zeta(b)<\infty$. It follows that, for each $y \in \mathscr{E}$ with $y<b, F_{0}(y, b)=$ $\lim _{z \rightarrow b} F_{0}(y, z)$ exists in $\overline{\mathbb{R}^{+}}$. The optimization of $F_{0}$ therefore includes the possibility $z=b$.

Next consider when $b<\infty$ is a natural boundary with $c_{0}(b)<\infty$ for which Condition 2.3(b)(ii) holds. Then, for each $y \in \mathcal{E} \backslash\{b\}, F_{0}(y, \cdot)$ is increasing for $z>z_{y}$ and, thus, the infimal value of $F_{0}$ is not obtained in the limit as $z \rightarrow b$.

Now assume that $b$ is a natural boundary for which $c_{0}(b)=\infty$. Case (ii) of the proof of Proposition 3.5 of Helmes et al. (2017) establishes that $F(y, b)=\infty$ for each $y \in \mathcal{E} \backslash\{b\}$. 
Case (iii): the vertex $(b, b)$ with b being natural. Using (2.4) of Lemma 2.1,

$$
\liminf _{(y, z) \rightarrow(b, b)} F_{0}(y, z) \geq \liminf _{(y, z) \rightarrow(b, b)} \frac{g_{0}(z)-g_{0}(y)}{\zeta(z)-\zeta(y)}=c_{0}(b) .
$$

Thus, the infimum does not occur in the limit as $(y, z) \rightarrow(b, b)$ since $(\tilde{y}, \tilde{z})$ of Condition 2.3(b)(ii) satisfies $F_{0}(\tilde{y}, \tilde{z})<c_{0}(b)$ or $c_{0}(b)=\infty$ in Condition 2.3(b)(i).

Case (iv): the boundary $y=a$, excluding $(a, b)$ when $b$ is natural. As shown in case (iv) of the proof of Proposition 3.5 of Helmes et al. (2017), when $a$ is a regular or an exit boundary, $F_{0}(a, z)$ exists in $\overline{\mathbb{R}^{+}}$for each $z \in \mathcal{E}$ with $z>a$. The optimization of $F_{0}$ therefore includes values with $y=a$. This proof also shows that, when $a$ is a natural boundary for which $c_{0}(a)=\infty$,

$$
\lim _{y \rightarrow a} F_{0}(y, z) \geq \lim _{y \rightarrow a} c_{0}(y)=\infty .
$$

Proposition 3.5 of Helmes et al. (2017) does not consider the case when $a>-\infty$ is a natural boundary with $c_{0}(a)<\infty$, so a proof is required. Since Condition 2.3(a)(ii) holds, $F_{0}(\cdot, z)$ is strictly decreasing for each $z \in \mathcal{E}$ and, hence, the infimal value of $F_{0}$ does not occur in the limit as $y \rightarrow a$.

Case (v): the vertex $(a, b)$ with both boundaries being natural. When $c_{0}(a)=\infty$ or $c_{0}(b)=\infty$, case (v) of the proof of Proposition 3.5 of Helmes et al. (2017) establishes that

$$
\lim _{(y, z) \rightarrow(a, b)} F_{0}(y, z)=\infty .
$$

We therefore need to consider models in which $a>-\infty$ and $b<\infty$ with $c_{0}(a), c_{0}(b)<\infty$. Note that both Condition 2.3(a)(ii) and (b)(ii) hold.

Choose $\varepsilon$ such that $0<\varepsilon<\left(c_{0}(a)-F_{0}(\hat{y}, \hat{z})\right) \wedge\left(c_{0}(b)-F_{0}(\tilde{y}, \tilde{z})\right)$. Again, Lemma 2.1 establishes that there exists some $y_{\varepsilon}, z_{\varepsilon} \in \ell$ such that

$$
\frac{g_{0}\left(y_{\varepsilon}\right)-g_{0}(y)}{\zeta\left(y_{\varepsilon}\right)-\zeta(y)} \geq c_{0}(a)-\varepsilon \quad \text { for all } y \in\left(a, y_{\varepsilon}\right)
$$

and

$$
\frac{g_{0}(z)-g_{0}\left(z_{\varepsilon}\right)}{\zeta(z)-\zeta\left(z_{\varepsilon}\right)} \geq c_{0}(b)-\varepsilon \text { for all } z \in\left(z_{\varepsilon}, b\right) .
$$

Thus, for each $y$ with $a<y<y_{\varepsilon}$ and $z$ such that $z_{\varepsilon}<z<b$, we have

$$
\begin{aligned}
F_{0}(y, z) \geq & \frac{g_{0}(z)-g_{0}(y)}{\zeta(z)-\zeta(y)} \\
= & \frac{g_{0}(z)-g_{0}\left(z_{\varepsilon}\right)+g_{0}\left(z_{\varepsilon}\right)-g_{0}\left(y_{\varepsilon}\right)+g_{0}\left(y_{\varepsilon}\right)-g_{0}(y)}{\zeta(z)-\zeta(y)} \\
= & \left(\frac{g_{0}(z)-g_{0}\left(z_{\varepsilon}\right)}{\zeta(z)-\zeta\left(z_{\varepsilon}\right)}\right)\left(\frac{\zeta(z)-\zeta\left(z_{\varepsilon}\right)}{\zeta(z)-\zeta(y)}\right)+\frac{g_{0}\left(z_{\varepsilon}\right)-g_{0}\left(y_{\varepsilon}\right)}{\zeta(z)-\zeta(y)} \\
& +\left(\frac{g_{0}\left(y_{\varepsilon}\right)-g_{0}(y)}{\zeta\left(y_{\varepsilon}\right)-\zeta(y)}\right)\left(\frac{\zeta\left(y_{\varepsilon}\right)-\zeta(y)}{\zeta(z)-\zeta(y)}\right) \\
\geq & {\left[c_{0}(b)-\varepsilon\right] \frac{\zeta(z)-\zeta\left(z_{\varepsilon}\right)}{\zeta(z)-\zeta(y)}+\frac{g_{0}\left(z_{\varepsilon}\right)-g_{0}\left(y_{\varepsilon}\right)}{\zeta(z)-\zeta(y)}+\left[c_{0}(a)-\varepsilon\right] \frac{\zeta\left(y_{\varepsilon}\right)-\zeta(y)}{\zeta(z)-\zeta(y)} } \\
\geq & {\left[\left(c_{0}(a)-\varepsilon\right) \wedge\left(c_{0}(b)-\varepsilon\right)\right] \frac{\zeta(z)-\zeta\left(z_{\varepsilon}\right)+\zeta\left(y_{\varepsilon}\right)-\zeta(y)}{\zeta(z)-\zeta(y)} . }
\end{aligned}
$$


Since $\lim _{(y, z) \rightarrow(a, b)}(\zeta(z)-\zeta(y))=\infty$, it follows that

$$
\liminf _{(y, z) \rightarrow(a, b)} F_{0}(y, z) \geq\left[\left(c_{0}(a)-\varepsilon\right) \wedge\left(c_{0}(b)-\varepsilon\right)\right]>F_{0}(\bar{y}, \bar{z}) \wedge F_{0}(\tilde{y}, \tilde{z}) .
$$

Therefore, the infimum of $F_{0}$ is not obtained in the limit as $(y, z) \rightarrow(a, b)$.

Case (vi): the vertex $(a, a)$ with a being natural. This argument is essentially the same as for the vertex $(b, b)$ using Lemma 2.1 and Condition 2.3(a)(ii).

In summary, we have established that the infimal value of $F_{0}$ does not occur at any boundary, so there exists some $\left(y_{0}^{*}, z_{0}^{*}\right) \in \mathcal{R}$ which minimizes the function $F_{0}$.

\section{Appendix B. Proofs for Section 5}

Proof of Lemma 5.1. Fix $n \in \mathbb{N}$ arbitrarily. To show that $G_{n}$ is bounded, note first that, on the set $\left\{x \in \mathcal{E}:\left|G_{0}(x)\right|<1\right\}$,

$$
\left|G_{n}(x)\right|=\frac{\left|G_{0}(x)\right|}{1+h\left(G_{0}(x)\right) / n}<1 .
$$

On the set $\left\{x \in \mathcal{E}:\left|G_{0}(x)\right| \geq 1\right\}$, we have

$$
\left|G_{n}(x)\right|=\frac{n}{\left(n /\left|G_{0}(x)\right|\right)+1} \leq n .
$$

Combining these estimates indicates that $\left|G_{n}(x)\right| \leq n$. In addition, when $a$ and $b$ are finite and natural, $G_{0}(x) \rightarrow \pm \infty$ implies that $\lim _{x \rightarrow a} G_{n}(x)=-n$ and $\lim _{x \rightarrow b} G_{n}(x)=n$, respectively, so we may define $G_{n}$ at such boundaries to be the appropriate limiting value.

Straightforward calculations establish that, for $x \in \mathcal{E}$,

$$
G_{n}^{\prime}(x)=\frac{G_{0}^{\prime}(x)\left[1+h\left(G_{0}(x)\right) / n-G_{0}(x) h^{\prime}\left(G_{0}(x)\right) / n\right]}{\left(1+h\left(G_{0}(x)\right) / n\right)^{2}},
$$

and

$$
\begin{aligned}
G_{n}^{\prime \prime}(x)= & \frac{G_{0}^{\prime \prime}(x)\left[1+h\left(G_{0}(x)\right) / n-G_{0}(x) h^{\prime}\left(G_{0}(x)\right) / n\right]}{\left(1+h\left(G_{0}(x)\right) / n\right)^{2}}-\frac{\left(G_{0}^{\prime}(x)\right)^{2} G_{0}(x) h^{\prime \prime}\left(G_{0}(x)\right)}{n\left(1+h\left(G_{0}(x)\right) / n\right)^{2}} \\
& -\frac{2\left(G_{0}^{\prime}(x)\right)^{2} h^{\prime}\left(G_{0}(x)\right)\left[1+h\left(G_{0}(x)\right) / n-G_{0}(x) h^{\prime}\left(G_{0}(x)\right) / n\right]}{n\left(1+h\left(G_{0}(x)\right) / n\right)^{3}} .
\end{aligned}
$$

Observe that

$$
\left(\sigma(x) G_{n}^{\prime}(x)\right)^{2}=\left(\frac{\left(\sigma(x) G_{0}^{\prime}(x)\right)^{2}}{\left(n+h\left(G_{0}(x)\right)\right)^{3}}\right)\left(\frac{n^{3}\left[1+h\left(G_{0}(x)\right) / n-G_{0}(x) h^{\prime}\left(G_{0}(x)\right) / n\right]^{2}}{1+h\left(G_{0}(x)\right) / n}\right) .
$$

By Condition 5.1 (and (5.3) when necessary), there exist $y_{1}>a, z_{1}<b$, and $L<\infty$ such that the first factor is bounded for all $x \in\left[y_{1}, z_{1}\right]^{\mathrm{c}}$. The continuity of this factor implies that it is also bounded on $\left[y_{1}, z_{1}\right]$. Since the second factor is bounded by $4 n^{3},\left(\sigma G_{n}^{\prime}\right)^{2}$ is bounded.

Next, we show that $A G_{n}$ is bounded. Using the expressions for $G_{n}^{\prime}$ and $G_{n}^{\prime \prime}$, it follows that

$$
\begin{aligned}
A G_{n}(x) & \\
= & \frac{A G_{0}(x)\left[1+h\left(G_{0}(x)\right) / n-G_{0}(x) h^{\prime}\left(G_{0}(x)\right) / n\right]}{\left(1+h\left(G_{0}(x)\right) / n\right)^{2}}-\frac{\left(\sigma(x) G_{0}^{\prime}(x)\right)^{2} G_{0}(x) h^{\prime \prime}\left(G_{0}(x)\right)}{2 n\left(1+h\left(G_{0}(x)\right) / n\right)^{2}} \\
& -\frac{\left(\sigma(x) G_{0}^{\prime}(x)\right)^{2} h^{\prime}\left(G_{0}(x)\right)\left[1+h\left(G_{0}(x)\right) / n-G_{0}(x) h^{\prime}\left(G_{0}(x)\right) / n\right]}{n\left(1+h\left(G_{0}(x)\right) / n\right)^{3}} \\
= & A G_{n}^{(1)}(x)+e_{n}^{(2)}(x)+e_{n}^{(3)}(x) .
\end{aligned}
$$

We examine these terms carefully. Recall that $A G_{0}(x)=F_{0}^{*}-c_{0}(x)$ for $x \in \ell$. 
Consider first the case in which $c_{0}(a)=\infty ; c_{0}(b)=\infty$ is handled similarly, so is omitted. Let $y_{1}>a$ be as in Condition 5.1(a)(i). For $x \leq y_{1}$ such that $\left|G_{0}(x)\right| \geq 1, h\left(G_{0}(x)\right)=\left|G_{0}(x)\right|$ and $h^{\prime \prime}\left(G_{0}(x)\right)=0$, so $A G_{n}^{(1)}(x)$ and $e_{n}^{(3)}(x)$ are uniformly bounded due to Condition 5.1(a)(i) while $e_{n}^{(2)}(x)=0$. For $x \leq y_{1}$ such that $\left|G_{0}(x)\right|<1$, Condition 5.1(a)(i) implies that both $c_{0}(x)$ and $\left(\sigma(x) G_{0}^{\prime}(x)\right)^{2}$ are uniformly bounded. Since $h(x)$ and $h^{\prime \prime}(x)$ are also uniformly bounded for $|x|<1$, it follows that $A G_{n}$ is bounded on $\left(a, y_{1}\right)$.

Now consider the case in which $c_{0}(a)<\infty$; again, the case $c_{0}(b)<\infty$ is handled similarly. The function $A G_{n}^{(1)}$ remains bounded on $\left[a, y_{1}\right)$ since continuity of $c_{0}$ at $a$ implies that there is some neighborhood $\left[a, y_{2}\right)$ of $a$ such that $\left|2\left(F_{0}^{*}-c_{0}(x)\right) /\left(1+h\left(G_{0}(x)\right) / n\right)^{2}\right| \leq 2 c_{0}(a)+$ $2 F_{0}^{*}+1$ for $x \in\left[a, y_{2}\right)$. When $y_{2}<y_{1}$, continuity implies that $A G_{n}^{(1)}$ remains bounded on $\left[y_{2}, y_{1}\right)$. Essentially the same analysis as above but using Condition 5.1(a)(ii) handles $e_{n}^{(2)}$ and $e_{n}^{(3)}$ on $\left(a, y_{1}\right)$. Thus, $A G_{n}$ is bounded in $\left(a, y_{1}\right)$ and this relation extends to include $x=a$.

Similar arguments with regard to the boundary $b$ show that $A G_{n}$ is bounded in $\left(z_{1}, b\right)$, extending to include $b$ under Condition 5.1(b)(ii) when $c_{0}(b)<\infty$. By continuity, $A G_{n}$ is bounded on $\left[y_{1}, z_{1}\right]$, establishing that $A G_{n}$ is bounded.

We now verify the boundary behaviour of Definition 5.2. Regarding $A G_{n}$, we must show that it is continuous at finite, natural boundaries for which $c_{0}$ is finite. We examine the boundary $a$; the analysis for $b$ is similar. If $\left|G_{0}(a)\right|<\infty$ then Condition 5.1(c) implies that $A G_{n}(a)=$ $\lim _{x \rightarrow a} A G_{n}(x)$ exists and is finite. Now consider the case in which $\left|G_{0}(a)\right|=\infty$. Then, for sufficiently small $x$ (without loss of generality $\left.x \leq y_{1}\right),\left|G_{0}(x)\right| \geq 1$, so $h\left(G_{0}(x)\right)=\left|G_{0}(x)\right|$ and $h^{\prime \prime}\left(G_{0}(x)\right)=0$. It then immediately follows that $e_{n}^{(2)}(x)=0$ while $A G_{n}^{(1)}(x)$ converges to 0 . For $e_{n}^{(3)}(x)$, Condition 5.1(a)(ii) implies that, for $x \leq y_{1}$,

$$
\frac{\left(\sigma(x) G_{0}^{\prime}(x)\right)^{2}}{\left(1+h\left(G_{0}(x)\right) / n\right)^{3}} \leq \frac{\left(\sigma(x) G_{0}^{\prime}(x)\right)^{2}}{\left(1+\left|G_{0}(x)\right| / n\right)^{2+\varepsilon}} \cdot \frac{1}{\left(1+\left|G_{0}(x)\right| / n\right)^{1-\varepsilon}},
$$

which again converges to 0 as $x \rightarrow a$. Thus, defining $A G_{n}(a)=0$ makes $A G_{n}$ continuous at $a$.

Now, consider the case of $a$ being a reflecting boundary. Using the definitions of $g_{0}, \zeta$, and $G_{0}$ in (2.2) and (4.1), respectively, it follows that

$$
G_{0}^{\prime}(x)=s(x) \int_{x}^{b} 2\left(c_{0}(v)-F_{0}^{*}\right) \mathrm{d} M(v) .
$$

Condition 5.1(c)(ii) then implies that $\left|G_{0}^{\prime}(a)\right|<\infty$. From the expression for $G_{n}^{\prime}(x)$ in (B.1), $\lim _{x \rightarrow a} G_{n}^{\prime}(x)$ exists and is finite.

Finally, when $a$ is a sticky boundary and $c_{0}(a)<\infty$, Condition 5.1(c)(i) along with (B.1) establishes that Definition 5.2(b)(iii) holds.

Proof of Lemma 5.2. We consider the convergence of $G_{n}$ first. For all $x \in \ell,\left|G_{0}(x)\right|<\infty$, so

$$
\lim _{n \rightarrow \infty} G_{n}(x)=\lim _{n \rightarrow \infty} \frac{G_{0}(x)}{1+h\left(G_{0}(x)\right) / n}=G_{0}(x) .
$$

This result holds also at the boundaries whenever $G_{0}$ is bounded there.

Now consider the case in which $\left|G_{0}(a)\right|=\lim _{x \rightarrow a}\left|G_{0}(x)\right|=\infty$. Observe that, for $x \in \ell$,

$$
G_{0}(x)=\int_{x_{0}}^{x} \int_{u}^{b} 2\left[c_{0}(v)-F_{0}^{*}\right] \mathrm{d} M(v) \mathrm{d} S(u) .
$$


When $a$ is attainable, $\zeta(a)>-\infty$, so if $c_{0}$ were bounded in a neighborhood of $a, G_{0}$ would also be bounded in the neighborhood. Thus, $c_{0}(x) \rightarrow \infty$ as $x \rightarrow a$ and, as a result, $G_{0}(a)=$ $\lim _{x \rightarrow a} G_{0}(x)=-\infty$. When $a$ is a natural boundary, $\zeta(a)=-\infty$ and Condition 2.3 implies that $c_{0}(a)>F_{0}^{*}$, so again $G_{0}(a)=-\infty$. Then regardless of the type of boundary, for $x$ sufficiently close to $a, G_{0}(x) \leq-1$. Since $h(x)=|x|$ on the set where $|x| \geq 1$,

$$
\lim _{x \rightarrow a} G_{n}(x)=\lim _{x \rightarrow a} \frac{G_{0}(x)}{1+\left|G_{0}(x)\right| / n}=-n=: G_{n}(a),
$$

so again $\lim _{n \rightarrow \infty} G_{n}(a)=-\infty=G_{0}(a)$.

A similar argument at the boundary $b$ establishes that $\lim _{n \rightarrow \infty} G_{n}(b)=G_{0}(b)$ and, therefore, $G_{n}$ converges pointwise to $G_{0}$. It is therefore immediate that $B G_{n}$ converges pointwise to $B G_{0}$ on $\overline{\mathcal{R}}$.

Turning to $A G_{n}$, recall from (B.2) that $A G_{n}=A G_{n}^{(1)}+e_{n}^{(2)}+e_{n}^{(3)}$. Since $G_{0} \in C^{2}(\ell)$, $G_{0}(x)$ and $G_{0}^{\prime}(x)$ are finite for $x \in \ell$. It then follows that $\lim _{n \rightarrow \infty} A G_{n}(x)=A G_{0}(x)$ for $x \in \ell$. A careful examination of the convergence at the boundaries is required.

Assume that $c_{0}$ is finite at the boundaries. When $\left|G_{0}(a)\right|<\infty$, Condition 5.1(c) implies that $\lim _{x \rightarrow a} \sigma(x) G_{0}^{\prime}(x)=K_{1}$ for some finite $K_{1}$. Denote this limit by $\sigma(a) G_{0}^{\prime}(a)$ and set $A G_{0}(a)=F_{0}^{*}-c_{0}(a)$. Then

$$
\lim _{x \rightarrow a} A G_{n}(x)=A G_{n}^{(1)}(a)+e_{n}^{(2)}(a)+e_{n}^{(3)}(a)=: A G_{n}(a) .
$$

As before, it follows that $\lim _{n \rightarrow \infty} A G_{n}(a)=A G_{0}(a)$. A similar analysis applies at the boundary $b$ when $\left|G_{0}(b)\right|<\infty$. Observe that, under the assumption that $c_{0}$ is finite at the boundaries, $G_{0}$ is bounded at the boundaries when $a$ is attainable and when $b$ is an entrance boundary.

Now consider the case in which $c_{0}(a)<\infty$ and $\left|G_{0}(a)\right|=\infty$. Thus, $a$ is a natural boundary and, hence, Condition 2.3 implies that $G_{0}(a)=-\infty$. Then, for some $y_{1}>a, G_{0}(x) \leq-1$ for all $a<x<y_{1}$ and, as a result, $h\left(G_{0}(x)\right)=\left|G_{0}(x)\right|$ and $h^{\prime \prime}\left(G_{0}(x)\right)=0$. Examining the expression for $A G_{n}$, we see that

$$
A G_{n}(x)=\frac{A G_{0}(x)}{\left(1+\left|G_{0}(x)\right| / n\right)^{2}}-\frac{\left(\sigma(x) G_{0}^{\prime}(x)\right)^{2} \operatorname{sgn}\left(G_{0}(x)\right)}{n\left(1+\left|G_{0}(x)\right| / n\right)^{3}} .
$$

Since $A G_{0}(x)=F_{0}^{*}-c_{0}(x), c_{0}(a)<\infty$, and $\left|G_{0}(a)\right|=\infty$, the first term converges to 0 as $x \rightarrow a$. Using Condition 5.1(a)(ii), we have

$$
\left|\frac{\left(\sigma(x) G_{0}^{\prime}(x)\right)^{2} \operatorname{sgn}\left(G_{0}(x)\right)}{n\left(1+\left|G_{0}(x)\right| / n\right)^{3}}\right| \leq \frac{L n^{2}}{\left(1+\left|G_{0}(x)\right|\right)^{1-\varepsilon}},
$$

so this term also converges to 0 as $x \rightarrow a$. Therefore, $A G_{n}(a)=0$, and it follows that $\lim _{n \rightarrow \infty} A G_{n}(a)=0>F_{0}^{*}-c_{0}(a)=A G_{0}(a)$. A similar argument using Condition 5.1(b)(ii) establishes that $A G_{n}(b) \geq A G_{0}(b)$ for each $n$ when $\left|G_{0}(b)\right|=\infty$.

\section{Appendix C. A counterintuitive example}

In this appendix we present an example of an inventory model and a particular ordering policy for which the long-term average cost is finite, and, hence, $\left\{\mu_{0, t_{j}}\right\}$ is tight for any $\left\{t_{j}\right\}$ with $t_{j} \rightarrow \infty$, but the corresponding sequence $\left\{\mu_{1, t_{j}}\right\}$ is not tight.

Consider the classical drifted Brownian motion inventory model of (6.1) in Section 6:

$$
X_{0}(t)=W(t)-t, \quad t \geq 0 ;
$$


for notational simplicity, the initial inventory level is $x_{0}=0$ and the drift and diffusion coefficients are $\mu=-1$ and $\sigma=1$. The cost functions of (6.2) are specified as

$$
c_{0}(x)=2|x| \quad \text { for all } x \in \mathbb{R}, \quad c_{1}(y, z)=k_{1}+(z-y) \quad \text { for all }(y, z) \in \overline{\mathcal{R}} .
$$

A special ordering policy $(\tau, Y)$ will now be described. It runs in cycles, each of which is composed of two phases. For cycle $i=1,2,3, \ldots$, phase 1 consists of using the $(0,1)$ ordering policy a total of $2^{i-1}$ times; the length of each subcycle is a random variable having mean 1 . Phase 2 involves a single $\left(0,2^{(i-1) / 2}\right)$-ordering policy followed immediately by using the $\left(2^{(i-1) / 2}, 2^{(i-1) / 2}\right)$-ordering policy $2^{i-1}$ times.

The formal description of the ordering policy is now given.

Definition C.1. (The policy $(\tau, Y)$.) For cycle $i=1$, define

$$
\begin{array}{llll}
\tau_{1,1}=0, & \tau_{1,2}=\inf \left\{t \geq \tau_{1,1}: X(t)=0\right\}, & \tau_{1,3}=\tau_{1,2}, \\
Y_{1,1}=1, & Y_{1,2}=1, & Y_{1,3}=0,
\end{array}
$$

and, for cycle $i=2,3,4, \ldots$, define the orders in phase 1 to be

$$
\begin{aligned}
\tau_{i, 1} & =\inf \left\{t \geq \tau_{i-1,2^{i}+1}: X(t)=0\right\}, & & \tau_{i, j}=\inf \left\{t \geq \tau_{i, j-1}: X(t)=0\right\}, \\
Y_{i, 1} & =1, & Y_{i, j} & =1,
\end{aligned}
$$

for $j=2, \ldots, 2^{i-1}$, and the orders in phase 2 by

$$
\begin{aligned}
\tau_{i, 2^{i-1}+1} & =\inf \left\{t \geq \tau_{i, 2^{i-1}}: X(t)=0\right\}, & & \tau_{i, j}=\tau_{i, 2^{i-1}+1}, \\
Y_{i, 2^{i-1}+1} & =2^{(i-1) / 2}, & & Y_{i, j}=0,
\end{aligned}
$$

for $j=2^{i-1}+2, \ldots, 2^{i}+1$.

Remark C.1. When formulating the ordering costs, traditionally no distinction is made between not ordering and ordering nothing, with no cost incurred in either case. In contrast, the formulation in this paper has orders of size 0 that incur the fixed cost $k_{1}$. The policy $(\tau, Y)$ uses 0 -size orders to create nontrivial masses in the average ordering measure $\mu_{1, t}$ at arbitrarily large values on the diagonal $z=y$ without affecting the length of phase 2, provided $t$ is sufficiently large. Under the traditional formulation, it is possible to place many orders of suitably small sizes, resulting in similar masses in neighborhoods near the diagonal at arbitrary distances from the origin (with large $t$ ), such that the length of phase 2 is barely increased. The analysis is essentially the same as in this paper but requires more careful bookkeeping without affecting the limiting results. The costly 0 -size orders considerably simplify the computations.

The main result of this appendix can now be stated.

Theorem C.1. For the drifted Brownian motion inventory model, let $(\tau, Y)$ be the ordering policy of Definition C.1, let $X$ be the resulting inventory process, and let $\left\{\mu_{0, t}\right\}$ and $\left\{\mu_{1, t}\right\}$ respectively be the corresponding average expected occupation and ordering measures defined in (3.1). Then

(a) $J_{0}(\tau, Y)<\infty$;

(b) $\left\{\mu_{0, t}: t>0\right\}$ is tight as $t \rightarrow \infty$; and

(c) $\left\{\mu_{1, t}: t>0\right\}$ is not tight.

Proof. This theorem is proven in pieces. Proposition C. 4 shows that the long-term average holding costs are bounded and, thus, an argument as in the proof of Proposition 3.1 establishes 
the tightness of the average expected occupation measures $\left\{\mu_{0, t}\right\}$ as $t \rightarrow \infty$. Finally, Proposition C. 5 shows both that the long-term average ordering costs are finite and that the average expected ordering measures $\left\{\mu_{1, t}\right\}$ are not tight.

Our analysis depends on a careful construction of the inventory process $X$ under the ordering policy $(\tau, Y)$ of Definition C.1. Independent copies of the diffusion $X_{0}$ of (6.1) are pieced together at the jump times (see, e.g. the appendix of Christensen (2014) for such a construction) with the implication that the various ordering subcycles are independent.

The initial analysis examines the long-term average cost of the $(\tau, Y)$ policy. It begins by focusing on the holding costs. Note that the only orders which affect the length of cycle $i$ are the $2^{i-1}$ times that the $(0,1)$ policy is used and the one time that the $\left(0,2^{(i-1) / 2}\right)$ policy occurs; the $2^{i-1}$ times that orders of size 0 are placed do not change the state of the inventory or lengthen the subcycles, so have no affect on the holding costs. Thus, it is sufficient to restrict the analysis solely to the nonzero orders.

Let $\delta:=\left\{\sigma_{1}, \sigma_{2}, \sigma_{3}, \ldots\right\}$ denote the times of the nonzero orders. Let $i \in \mathbb{N}$ denote the cycle and $j \in\left\{1,2, \ldots, 2^{i-1}+1\right\}$ be the number of the nonzero order within cycle $i$. Then observe that $\sigma_{n}=\tau_{i, j}$, where $n=2^{i-1}+i+j-2$. The ensuing computations are simplified by a shift in the index for $j$. For $i \geq 2$, define $\tau_{i, 0}=\tau_{i-1,2^{i-1}+1}$, so that order number 'zero' of the $i$ th cycle is the last nonzero order, in fact the large order, of cycle $(i-1)$.

Our first result gives a strong law of large numbers result for the cycle lengths.

Proposition C.1. Let $(\tau, Y)$ be the ordering policy of Definition C.1, and let $\&$ be the times of the nonzero orders. Then

$$
\lim _{n \rightarrow \infty} \frac{\sigma_{n}}{n}=1 \text { a.s. }
$$

Proof. Define the independent (but not identically distributed) random variables

$$
\beta_{0}=\sigma_{1}=0 \quad \text { and } \quad \beta_{k}=\sigma_{k+1}-\sigma_{k}, \quad k \in \mathbb{N} ;
$$

thus, $\beta_{k}$ gives the random length of time of the $k$ th interorder interval. Then $\sigma_{n}=\sum_{k=0}^{n-1} \beta_{k}, n \in \mathbb{N}$.

Note that, apart from $\beta_{0}=0, \beta_{n}$ is either the length of a $(0,1)$ subcycle or a $\left(0,2^{(i-1) / 2}\right)$ subcycle. More precisely, for each $i \geq 1, \beta_{2^{i}+i-1}$ is the length of the cycle arising from the $\left(0,2^{(i-1) / 2}\right)$ subcycle and, for $n \neq 2^{i}-1+i, \beta_{n}$ is the length of a $(0,1)$ subcycle. Using the Laplace transform of the hitting time of a drifted Brownian motion process (see Formula 2.0.1 of Borodin and Salminen (2002, p. 295)), we can determine that

$$
\begin{gathered}
\mathbb{E}\left[\beta_{2^{i-1}+i+j-2}\right]=1 \text { and } \operatorname{var}\left(\beta_{2^{i-1}+i+j-2}\right)=1 \text { for } j=1,2, \ldots, 2^{i-1}, i \geq 1, \\
\mathbb{E}\left[\beta_{2^{i}+i-1}\right]=2^{(i-1) / 2} \text { and } \operatorname{var}\left(\beta_{2^{i}+i-1}\right)=2^{(i-1) / 2} \text { for } i \geq 1 .
\end{gathered}
$$

Next observe that

$$
\begin{aligned}
\sum_{n=2}^{\infty} \frac{\operatorname{var}\left(\beta_{n}\right)}{n^{2}} & =\sum_{i=2}^{\infty} \sum_{j=0}^{2^{i-1}} \frac{\operatorname{var}\left(\beta_{2^{i-1}+i+j-2}\right)}{\left(2^{i-1}+i+j-2\right)^{2}} \\
& \leq \sum_{i=1}^{\infty} \sum_{j=1}^{2^{i-1}} \frac{1}{\left(2^{i-1}+i+j-2\right)^{2}}+\sum_{i=1}^{\infty} \frac{2^{(i-1) / 2}}{\left(2^{i-1}+i-1\right)^{2}} \\
& \leq \sum_{n=1}^{\infty} \frac{1}{n^{2}}+\sum_{i=1}^{\infty} \frac{1}{2^{3(i-1) / 2}} \\
& <\infty
\end{aligned}
$$

By Kolmogorov's strong law of large numbers (cf. Theorem 2 of Shiryaev (1996, p. 389)), it 
follows that

$$
\lim _{n \rightarrow \infty}\left(\frac{1}{n} \sum_{k=1}^{n} \beta_{k}-\bar{\mu}_{n}\right)=0 \quad \text { a.s. }
$$

in which $\bar{\mu}_{n}=(1 / n) \sum_{k=1}^{n} \mathbb{E}\left[\beta_{k}\right]$. Note that $\sigma_{n+1}=\sum_{k=0}^{n} \beta_{k}=\sum_{k=1}^{n} \beta_{k}$. Thus, the above equation can be rewritten as $\lim _{n \rightarrow \infty}\left(\sigma_{n+1} / n-\bar{\mu}_{n}\right)=0$ a.s., which in turn implies (C.1) if we can show that $\bar{\mu}_{n} \rightarrow 1$ as $n \rightarrow \infty$.

We now analyze the convergence of $\bar{\mu}_{n}$. Again, for $n \geq 2$, write $n=2^{i-1}+i+j-2$ with $i \geq 2$ and $0 \leq j \leq 2^{i-1}$. Note that, for $1 \leq k \leq i-1$, cycle $k$ contains $2^{k-1}$ subcycles generated by $(0,1)$ ordering policies having a mean length of 1 and a single $\left(0,2^{(k-1) / 2}\right)$ subcycle with mean length $2^{(k-1) / 2}$, while the partial cycle $i$ has $j$ subcycles from $(0,1)$, Thus, for $n \geq 2$,

$$
\begin{aligned}
\frac{1}{n} \sum_{k=1}^{n} \mathbb{E}\left[\beta_{k}\right] & =\frac{1}{2^{i-1}+i+j-2}\left(\sum_{k=1}^{i-1}\left(2^{k-1}+2^{(k-1) / 2}\right)+j\right) \\
& =\frac{1}{2^{i-1}+i+j-2}\left(2^{i-1}-1+\frac{2^{(i-1) / 2}-1}{2^{1 / 2}-1}+j\right) \\
& =\left(1-\frac{1}{2^{i-1}+j}+\frac{1}{2^{1 / 2}-1} \frac{2^{(i-1) / 2}-1}{2^{i-1}+j}\right)\left(1+\frac{i-2}{2^{i-1}+j}\right)^{-1} .
\end{aligned}
$$

Obviously, we have

$$
\lim _{i \rightarrow \infty} \frac{1}{2^{i-1}+j}=\lim _{i \rightarrow \infty} \frac{2^{(i-1) / 2}-1}{2^{i-1}+j}=\lim _{i \rightarrow \infty} \frac{i-2}{2^{i-1}+j}=0 \quad \text { for each } j=0,1, \ldots, 2^{i-1} .
$$

Therefore, as $n \rightarrow \infty$ (and, hence, $i \rightarrow \infty),(1 / n) \sum_{k=1}^{n} \mathbb{E}\left[\beta_{k}\right]$ converges to 1 .

For this model, we now establish a variant of the elementary renewal theorem; the fact that the cycles are not identically distributed means that the theorem cannot simply be applied.

Proposition C.2. For $t \geq 0$, let $N(t)=\sum_{i=1}^{\infty} \mathbf{1}_{\left\{\sigma_{i} \leq t\right\}}=\max \left\{n: \sigma_{n} \leq t\right\}$ be the number of orders of positive size by time $t$. Then

$$
\lim _{t \rightarrow \infty} \frac{N(t)}{t}=1 \text { a.s. and in } L^{1} \text {. }
$$

Proof. First, by the definition of $N(t), \sigma_{N(t)} \leq t<\sigma_{N(t)+1}$ for each $t \geq 0$. Thus, for each $t \geq 0$,

$$
\frac{\sigma_{N(t)}}{N(t)} \leq \frac{t}{N(t)}<\frac{\sigma_{N(t)+1}}{N(t)}=\frac{N(t)+1}{N(t)} \frac{\sigma_{N(t)+1}}{N(t)+1} .
$$

As $t \rightarrow \infty$, Proposition C. 1 implies first that $N(t) \rightarrow \infty$ (a.s.) and then establishes the almostsure convergence of (C.2). In order to prove the $L^{1}$ convergence of (C.2), it is necessary to show that the collection $\{N(t) / t: t \geq 1\}$ is uniformly integrable. By Lemma 3 of Shiryaev (1996, p. 190), it suffices to show that $\sup _{t \geq 1} \mathbb{E}\left[(N(t) / t)^{2}\right]<\infty$. To this end, define the ordering policy $(\tilde{\tau}, \tilde{Y})$ which always uses the $(0,1)$ policy and denote by $\left\{\tilde{\sigma}_{n}: n \in \mathbb{N}\right\}$ the ordering times. Define the corresponding renewal process $\tilde{N}$ by $\tilde{N}(t)=\max \left\{n: \tilde{\sigma}_{n} \leq t\right\}=\sum_{i=1}^{\infty} \mathbf{1}_{\left\{\tilde{\sigma}_{i} \leq t\right\}}$. It then follows that $N(t) \leq \tilde{N}(t)$ for each $t \geq 0$ and, hence, $\mathbb{E}\left[(N(t) / t)^{2}\right] \leq \mathbb{E}\left[(\tilde{N}(t) / t)^{2}\right]$. Using a standard renewal argument (see, e.g. the proof of Theorem 5.5.2 of Chung (2001, pp. 143144)), it follows that $\mathbb{E}\left[(\tilde{N}(t) / t)^{2}\right]$ is uniformly bounded for $t \geq 1$, establishing the uniform integrability of $\{N(t) / t: t \geq 1\}$ and, hence, the $L^{1}$ convergence.

The next step on the way to showing that $J_{0}(\tau, Y)<\infty$ is to analyze the holding costs over a single cycle. Observe that $X\left(\sigma_{i}\right)$ is either 1 or $2^{(k-1) / 2}$ for some $k$; to simplify notation, let 
$z$ represent either value. Next $X(t)=z-\left(t-\sigma_{i}\right)+W\left(t-\sigma_{i}\right)$ for $t \in\left[\sigma_{i}, \sigma_{i+1}\right)$ since no orders are placed on the interval $\left(\sigma_{i}, \sigma_{i+1}\right)$ and, by the definition of $\sigma_{i+1}, z-\left(\sigma_{i+1}-\sigma_{i}\right)+$ $W\left(\sigma_{i+1}-\sigma_{i}\right)=0$. Again, to simplify notation, make the change of time $s=t-\sigma_{i}$ and define $\tau=\sigma_{i+1}-\sigma_{i}$. Thus, $X$ satisfies $X(s)=z-s+W(s)$ for $s \in[0, \tau)$.

Define

$$
\Theta_{\tau}=\int_{0}^{\tau} c_{0}(X(s)) \mathrm{d} s
$$

Then, by Proposition 2.6 of Helmes et al. (2017), it follows that $\mathbb{E}\left[\Theta_{\tau}\right]=z^{2}+z$.

We now establish a result similar to the law of large numbers for the holding costs.

Proposition C.3. Let $(\tau, Y)$ be given by Definition $C .1$, and let $X$ be the resulting inventory process. Then

$$
\limsup _{n \rightarrow \infty} \frac{1}{n} \sum_{k=1}^{n} \mathbb{E}\left[\int_{\sigma_{k}}^{\sigma_{k+1}} c_{0}(X(s)) \mathrm{d} s\right] \leq 3
$$

Proof. For simplicity of notation, define $\Theta_{k}:=\int_{\sigma_{k}}^{\sigma_{k+1}} c_{0}(X(s)) \mathrm{d} s$ for $k=1,2, \ldots$ As in the proof of Proposition C.1, write the index $n$ as $n=2^{i-1}+i+j-2$ with $i=2,3, \ldots$ and $j=0,1, \ldots, 2^{i-1}$. Recall that the large orders have indices with $j=0$ and, hence, $n=2^{i-1}+i-2$ for $i \geq 2$, so the formula for $\mathbb{E}\left[\Theta_{\tau}\right]$ above establishes that

$$
\mathbb{E}\left[\Theta_{2^{i-1}+i+j-2}\right]=2, \quad j=1, \ldots, 2^{i-1}, \quad \mathbb{E}\left[\Theta_{2^{i}+i-1}\right]=2^{i-1}+2^{(i-1) / 2}, \quad i \in \mathbb{N} .
$$

Consequently, for $n \geq 2$,

$$
\begin{aligned}
\frac{1}{n} \sum_{\ell=1}^{n} \mathbb{E}\left[\Theta_{\ell}\right] & =\frac{1}{2^{i-1}+i+j-2}\left(\sum_{k=1}^{i-1}\left(2^{k-1} \cdot 2+2^{k-1}+2^{(k-1) / 2}\right)+j \cdot 2\right) \\
& =\frac{1}{2^{i-1}+i+j-2}\left(3\left(2^{i-1}-1\right)+\frac{2^{(i-1) / 2}-1}{2^{1 / 2}-1}+2 j\right) \\
& \leq \frac{3\left(2^{i-1}+j\right)+\left(2^{(i-1) / 2}-1\right) /\left(2^{1 / 2}-1\right)-3}{2^{i-1}+i+j-2} \\
& =\left(3+\frac{1}{2^{1 / 2}-1} \frac{2^{(i-1) / 2}-1}{2^{i-1}+j}-\frac{3}{2^{i-1}+j}\right)\left(1+\frac{i-2}{2^{i-1}+j}\right)^{-1} .
\end{aligned}
$$

Using similar computations as those in the end of the proof of Proposition C.1, we see immediately that this ratio converges to 3 as $n \rightarrow \infty$ and, hence, $i \rightarrow \infty$. This gives (C.3) as desired.

We now parlay the asymptotics relative to cycles to verify that the long-term average holding costs related to $(\tau, Y)$ are finite.

Proposition C.4. Let $(\tau, Y)$ be given by Definition C.1, and let $X$ be the resulting inventory process. Then

$$
\limsup _{t \rightarrow \infty} \frac{1}{t} \mathbb{E}\left[\int_{0}^{t} c_{0}(X(s)) \mathrm{d} s\right] \leq 3 .
$$

Proof. Again, denote the nonzero ordering times by $\&$ and observe that the orders of size 0 do not affect the cycle lengths. Again, for each $t \geq 0$, recall that $N(t)=\max \left\{n: \sigma_{n} \leq t\right\}$, so that $\sigma_{N(t)} \leq t<\sigma_{N(t)+1}$. Thus, for positive $t$,

$$
\frac{1}{t} \int_{0}^{t} c_{0}(X(s)) \mathrm{d} s \leq \frac{1}{t} \int_{0}^{\sigma_{N(t)+1}} c_{0}(X(s)) \mathrm{d} s \leq \frac{1}{t} \int_{0}^{\sigma_{N(t)+2}} c_{0}(X(s)) \mathrm{d} s=\frac{1}{t} \sum_{j=1}^{N(t)+1} \Theta_{j},
$$


where, as in the proof of Proposition C.3, we used the notation $\Theta_{j}=\int_{\sigma_{j}}^{\sigma_{j+1}} c_{0}(X(s)) \mathrm{d} s$ for each $j \in \mathbb{N}$. Noting that $\{N(t)=0\}=\varnothing$ for each $t$, consider the expectation of the right-hand-side term above:

$$
\begin{aligned}
\mathbb{E}\left[\sum_{j=1}^{N(t)+1} \Theta_{j}\right] & =\mathbb{E}\left[\sum_{k=1}^{\infty} \mathbf{1}_{\{N(t)=k\}} \sum_{j=1}^{k+1} \Theta_{j}\right] \\
& =\mathbb{E}\left[\sum_{j=1}^{\infty} \Theta_{j} \sum_{k=j-1}^{\infty} \mathbf{1}_{\{N(t)=k\}}\right] \\
& =\sum_{j=1}^{\infty} \mathbb{E}\left[\Theta_{j} \mathbf{1}_{\{N(t) \geq j-1\}}\right] .
\end{aligned}
$$

Observe that $\{N(t) \geq j-1\}=\{N(t)<j-1\}^{\mathrm{c}}=\left\{\sigma_{j-1}>t\right\}^{\mathrm{c}}$ is independent of the process over the interval $\left[\sigma_{j}, \sigma_{j+1}\right)$. Therefore,

$$
\begin{aligned}
\sum_{j=1}^{\infty} \mathbb{E}\left[\Theta_{j} \mathbf{1}_{\{N(t) \geq j-1\}}\right] & =\sum_{j=1}^{\infty} \mathbb{E}\left[\Theta_{j}\right] \mathbb{E}\left[\mathbf{1}_{\{N(t) \geq j-1\}}\right] \\
& =\sum_{j=1}^{\infty} \mathbb{E}\left[\Theta_{j}\right]\left(\sum_{k=j-1}^{\infty} \mathbb{P}(N(t)=k)\right) \\
& =\sum_{k=1}^{\infty} \sum_{j=1}^{k+1} \mathbb{E}\left[\Theta_{j}\right] \mathbb{P}(N(t)=k) .
\end{aligned}
$$

By Proposition C.3, $\lim \sup _{k \rightarrow \infty}(1 / k) \sum_{j=1}^{k} \mathbb{E}\left[\Theta_{j}\right] \leq 3$, so, for any $\varepsilon>0$, there exists some $K_{0}<\infty$ such that $(1 /(k+1)) \sum_{j=1}^{k+1} \mathbb{E}\left[\Theta_{j}\right]<3+\varepsilon$ for all $k \geq K_{0}$. Thus,

$$
\begin{aligned}
& \sum_{k=1}^{\infty} \sum_{j=1}^{k+1} \mathbb{E}\left[\Theta_{j}\right] \mathbb{P}(N(t)=k) \\
& \quad=\sum_{k=1}^{K_{0}-1} \sum_{j=1}^{k+1} \mathbb{E}\left[\Theta_{j}\right] \mathbb{P}(N(t)=k)+\sum_{k=K_{0}}^{\infty}\left(\frac{1}{k+1} \sum_{j=1}^{k+1} \mathbb{E}\left[\Theta_{j}\right]\right)(k+1) \mathbb{P}(N(t)=k) \\
& \quad \leq \sum_{k=1}^{K_{0}-1} \sum_{j=1}^{k+1} \mathbb{E}\left[\Theta_{j}\right] \mathbb{P}(N(t)=k)+\sum_{k=K_{0}}^{\infty}(3+\varepsilon)(k+1) \mathbb{P}(N(t)=k) \\
& \quad \leq \sum_{k=1}^{K_{0}-1} \sum_{j=1}^{k+1} \mathbb{E}\left[\Theta_{j}\right]+(3+\varepsilon) \mathbb{E}[N(t)]+3+\varepsilon
\end{aligned}
$$

Since the first and last summands are constant, combining these upper bounds, dividing by $t$, and using Proposition C.2 yields

$$
\limsup _{t \rightarrow \infty} \frac{1}{t} \mathbb{E}\left[\int_{0}^{t} c_{0}(X(s)) \mathrm{d} s\right] \leq \lim _{t \rightarrow \infty} \frac{(3+\varepsilon) \mathbb{E}[N(t)]}{t}=3+\varepsilon .
$$

The result now follows since $\varepsilon>0$ is arbitrary. 
The final task is to verify that the long-term average ordering costs are finite and that $\left\{\mu_{1, t}\right\}$ is not tight as $t \rightarrow \infty$. The next proposition addresses both of these concerns since the analysis is very similar.

Proposition C.5. Let $(\tau, Y)$ be given by Definition C.1 for the drifted Brownian motion inventory model. For $t>0$, define $\mu_{1, t}$ by (3.1). Then

$$
\limsup _{t \rightarrow \infty} \int c_{1}(y, z) \mu_{1, t}(\mathrm{~d} y \times \mathrm{d} z) \leq 3 k_{1}+2
$$

and $\left\{\mu_{1, t}: t>1\right\}$ is not tight.

Proof. We first address the lack of tightness for $\left\{\mu_{1, t}\right\}$. Let $\Gamma \subset \overline{\mathcal{R}}$ be any compact set. Then there exists some $N_{0}$ such that, for all $i \geq N_{0},\left(2^{(i-1) / 2}, 2^{(i-1) / 2}\right) \in \Gamma^{\mathrm{c}}$.

Again, denote the times of nonzero orders by $s=\left\{\sigma_{n}: n \in \mathbb{N}\right\}$ and, for $n \geq 2$, write $n=2^{i-1}+i+j-2$ with $i \geq 2$ and $j=0, \ldots, 2^{i-1}$. Recall that the 'large' order of size $2^{(i-1) / 2}$ occurs at time $\sigma_{2^{i}+i-1}$. Under policy $(\tau, Y)$, there are a further $2^{i-1}$ orders of size 0 at time $\sigma_{2^{i}+i-1}$; denote this common time of ordering by $\tilde{\sigma}_{2^{i}+i-1, j}$ for $j=1, \ldots, 2^{i-1}$ for each of the 0 -size orders in cycle $i$. Thus,

$$
\begin{aligned}
& \mu_{1, t}\left(\Gamma^{\mathrm{c}}\right)=\frac{1}{t} \mathbb{E}\left[\sum_{n=1}^{\infty} \mathbf{1}_{\left\{\sigma_{n} \leq t\right\}} \mathbf{1}_{\Gamma^{\mathrm{c}}\left(X\left(\sigma_{n}-\right), X\left(\sigma_{n}\right)\right)}\right.\left.+\sum_{i=1}^{\infty} \sum_{j=1}^{2^{i-1}} \mathbf{1}_{\left\{\tilde{\sigma}_{2^{i}+i-1, j} \leq t\right\}} \mathbf{1}_{\Gamma^{\mathrm{c}}}\left(X\left(\tilde{\sigma}_{2^{i}+i-1, j}-\right), X\left(\tilde{\sigma}_{2^{i}+i-1, j}\right)\right)\right] \\
& \geq \frac{1}{t} \mathbb{E}\left[\sum_{i=N_{0}}^{\infty} \sum_{j=1}^{2^{i-1}} \mathbf{1}_{\left\{\tilde{\sigma}_{2^{i}+i-1, j} \leq t\right\}} \mathbf{1}_{\left.\Gamma^{\mathrm{c}}\left(2^{(i-1) / 2}, 2^{(i-1) / 2}\right)\right]}\right] \\
&=\frac{1}{t} \mathbb{E}\left[\sum_{i=N_{0}}^{\infty} 2^{i-1} \mathbf{1}_{\left\{\sigma_{2^{i}+i-1} \leq t\right\}}\right] .
\end{aligned}
$$

For each $t \geq 0$, define the processes $I$ and $J$ such that $N(t)=2^{I(t)-1}+I(t)+J(t)-2$ in which $I(t)$ denotes the cycle in which order $N(t)$ occurs and $0 \leq J(t) \leq 2^{I(t)-1}$. Since $N(t)$ is the number of nonzero orders placed by time $t \geq 0$, order number $N(t)$ is the $J(t)$ th order within cycle $I(t)$; again, $J(t)=0$ corresponds to the large order of the previous cycle. Using the processes $I$ and $J$, it follows that, for $0 \leq J(t) \leq 2^{I(t)-1}$,

$$
\begin{aligned}
\mu_{1, t}\left(\Gamma^{\mathrm{c}}\right) & \geq \frac{1}{t} \mathbb{E}\left[\sum_{i=N_{0}}^{\infty} 2^{i-1} \mathbf{1}_{\left\{\sigma_{2^{i}+i-1} \leq t\right\}}\right] \\
& \geq \frac{1}{t} \mathbb{E}\left[\sum_{\ell=2^{N_{0}+N_{0}-1}}^{I(t)-1} 2^{\ell-1}\right] \\
& =\frac{1}{t} \mathbb{E}\left[\sum_{\ell=1}^{I(t)-1} 2^{\ell-1}-\sum_{\ell=1}^{2^{N_{0}}+N_{0}-2} 2^{\ell-1}\right] \\
& =\frac{1}{t}\left(\mathbb{E}\left[2^{I(t)-1}\right]-2^{2^{N_{0}}+N_{0}-2}\right) .
\end{aligned}
$$


By Lemma C.2, $N(t) \rightarrow \infty$ (a.s.) as $t \rightarrow \infty$, so $I(t) \rightarrow \infty$ as well. Thus, the asymptotics of $\mu_{1, t}\left(\Gamma^{\mathrm{c}}\right)$ are determined by the asymptotics of the first summand above.

We next determine bounds on $I(t)$. Since $J(t) \leq 2^{I(t)-1}$ and $N(t)=2^{I(t)-1}+I(t)+$ $J(t)-2$, we have $N(t) \leq 2^{I(t)}+I(t)-2$ and, hence,

$$
2^{I(t)-1} \geq \frac{1}{2}(N(t)-I(t)+2) .
$$

Since $I(t) \geq 1$ and $J(t) \geq 0, N(t) \geq 2^{I(t)-1}-1$, so

$I(t)-1<\log _{2}(N(t)+1) \leq \log _{2}(N(t)+N(t))=\log _{2}\left(\frac{N(t) \cdot 2 t}{t}\right)=\log _{2}\left(\frac{N(t)}{t}\right)+\log _{2}(2 t)$.

Using this estimate in (C.6) yields

$$
2^{I(t)-1}>\frac{1}{2}\left(N(t)-\log _{2}\left(\frac{N(t)}{t}\right)-\log _{2}(2 t)+1\right) .
$$

Employing this lower bound in (C.5) and Jensen's inequality on the second summand, we have

$$
\mu_{1, t}\left(\Gamma^{\mathrm{c}}\right) \geq \frac{1}{2}\left(\frac{\mathbb{E}[N(t)]}{t}-\frac{\log _{2}(\mathbb{E}[N(t) / t])}{t}-\frac{\log _{2}(2 t)}{t}\right) \rightarrow \frac{1}{2} \quad \text { as } t \rightarrow \infty ;
$$

note that we have also used Proposition C. 2 on the second summand. Therefore, for any $\varepsilon<\frac{1}{2}$, $\mu_{1, t}\left(\Gamma^{\mathrm{c}}\right)>\varepsilon$ for all sufficiently large $t$. Hence, $\left\{\mu_{1, t}\right\}$ is not tight as $t \rightarrow \infty$.

Consider now the total ordering costs by time $t>0$. First, denote $(\tau, Y)=\left\{\left(\tau_{k}, Y_{k}\right): k \in \mathbb{N}\right\}$ to capture all of the orders. Next, denote the nonzero orders by $\delta$ and, as above, for each $i \in \mathbb{N}$ and $j=1, \ldots, 2^{i-1}$, let $\tilde{\sigma}_{2^{i}+i-1, j}=\sigma_{2^{i}+i-1}$ be the common time of the 0 -size orders in cycle $i$.

Since $t$ is finite and $0 \leq J(t) \leq 2^{I(t)-1}$,

$$
\begin{aligned}
& \mathbb{E}\left[\sum_{k=1}^{\infty}\right.\left.\mathbf{1}_{\left\{\tau_{k} \leq t\right\}} c_{1}\left(X\left(\tau_{k}-\right), X\left(\tau_{k}\right)\right)\right] \\
& \quad= \mathbb{E}\left[\sum_{i=1}^{I(t)-1}\left[2^{i-1}\left(k_{1}+1\right)+\left(k_{1}+2^{(i-1) / 2}\right)+2^{i-1} k_{1}\right]+J(t)\left(k_{1}+1\right)\right] \\
& \quad \leq \mathbb{E}\left[\left(\left(2 k_{1}+1\right)\left(2^{I(t)-1}-1\right)+\frac{2^{(I(t)-1) / 2}-1}{2^{1 / 2}-1}+k_{1}(I(t)-1)\right)+2^{I(t)-1}\left(k_{1}+1\right)\right] \\
& \quad=\mathbb{E}\left[\left(3 k_{1}+2\right) 2^{I(t)-1}+\frac{2^{(I(t)-1) / 2}-1}{2^{1 / 2}-1}+k_{1} I(t)-3 k_{1}-1\right] .
\end{aligned}
$$

Since $I(t) \geq 1$ and $J(t) \geq 0$, it follows that $2^{I(t)-1} \leq N(t)+1$ and so $2^{(I(t)-1) / 2} \leq$ $(N(t)+1)^{1 / 2}$. Using (C.7), we also have $I(t)<\log _{2}(N(t) / t)+\log _{2}(2 t)+1$. Then it follows from Jensen's inequality that

$$
\begin{aligned}
& \mathbb{E}\left[\sum_{k=1}^{\infty} \mathbf{1}_{\left\{\tau_{k} \leq t\right\}} c_{1}\left(X\left(\tau_{k}-\right), X\left(\tau_{k}\right)\right)\right] \\
& \quad \leq\left(3 k_{1}+2\right) \mathbb{E}[N(t)]+\frac{\mathbb{E}\left[(N(t)+1)^{1 / 2}\right]-1}{2^{1 / 2}-1}+k_{1} \mathbb{E}\left[\log _{2}\left(\frac{N(t)}{t}\right)+\log _{2}(2 t)+1\right]+1 \\
& \quad \leq\left(3 k_{1}+2\right) \mathbb{E}[N(t)]+\frac{(\mathbb{E}[N(t)+1])^{1 / 2}-1}{2^{1 / 2}-1}+k_{1}\left[\log _{2}\left(\mathbb{E}\left[\frac{N(t)}{t}\right]\right)+\log _{2}(2 t)+1\right]+1 .
\end{aligned}
$$

Now divide both sides by $t$, and then send $t \rightarrow \infty$, obtaining (C.4) from Proposition C.2. 
Remark C.2. (Final comments.) Under further analysis, we can obtain more precise results about the costs related to the ordering policy $(\tau, Y)$ of Definition C.1. As in the analysis of Proposition C.3, a lower bound on the limit inferior of the Cesàro mean of the expected cycle costs can be shown to be $\frac{5}{2}$. With more extensive calculations including the variance of the holding costs per cycle, these bounds can be shown to be tight and, moreover, that

$$
\liminf _{t \rightarrow \infty} \frac{1}{t} \int_{0}^{t} c_{0}(X(s)) \mathrm{d} s=\frac{5}{2} \quad \text { a.s. } \quad \limsup _{t \rightarrow \infty} \frac{1}{t} \int_{0}^{t} c_{0}(X(s)) \mathrm{d} s=3 \quad \text { a.s. }
$$

\section{Acknowledgements}

This research was supported in part by the Simons Foundation (under grant award numbers 246271 and 523736) and the NSFC (under grand number 11671034). The authors would like to thank the referees and editors for many useful comments and suggestions.

\section{References}

Bather, J. A. (1966). A continuous time inventory model. J. Appl. Prob. 3, 538-549.

Bensoussan, A. (2011). Dynamic Programming and Inventory Control (Stud. Prob. Optimization Statist. 3). IOS Press, Amsterdam.

Borodin, A. N. And SAlminen, P. (2002). Handbook of Brownian Motion-Facts and Formulae, 2nd edn. Birkhäuser, Basel.

Chen, H., Wu, O. Q. And Yao, D. D. (2010). On the benefit of inventory-based dynamic pricing strategies. Prod. Operat. Manag. 19, 249-260.

Christensen, S. (2014). On the solution of general impulse control problems using superharmonic functions. Stoch. Process. Appl. 124, 709-729.

Chung, K. L. (2001). A Course in Probability Theory, 3rd edn. Academic Press, San Diego, CA.

DAI, J. G. AND YAO, D. (2013). Brownian inventory models with convex holding cost, part 1: average-optimal controls. Stoch. Systems 3, 442-499.

He, S., YAO, D. AND ZHANG, H. (2017). Optimal ordering policy for inventory systems with quantity-dependent setup costs. Math. Operat. Res. 42, 979-1006.

Helmes, K. L., Stockbridge, R. H. and Zhu, C. (2017). Continuous inventory models of diffusion type: long-term average cost criterion. Ann. Appl. Prob. 27, 1831-1885.

Helmes, K. L., Stockbridge, R. H. And Zhu, C. (2018). Feller's branching diffusion inventory model. Supplementary material. Available at http://doi.org/10.1017/apr.2018.50.

Karlin, S. and Taylor, H. M. (1981). A Second Course in Stochastic Processes. Academic Press, New York.

Shiryaev, A. N. (1996). Probability (Graduate Texts Math. 95), 2nd edn. Springer, New York.

Sigman, K. AND WolfF, R. W. (1993). A review of regenerative processes. SIAM Rev. 35, 269-288.

Sulem, A. (1986). A solvable one-dimensional model of a diffusion inventory system. Math. Operat. Res. 11, $125-133$.

YAO, D., ChaO, X. And Wu, J. (2015). Optimal control policy for a Brownian inventory system with concave ordering cost. J. Appl. Prob. 52, 909-925. 